\title{
Chemistry of the Colloidal Group II-VI Nanocrystal Synthesis
}

\author{
by \\ Haitao Liu \\ B.S. (University of Science and Technology of China) 2001 \\ A dissertation submitted in partial satisfaction of the \\ requirements for the degree of \\ Doctor of Philosophy \\ in \\ Chemistry \\ in the \\ Graduate Division \\ of the \\ University of California, Berkeley \\ Committee in charge: \\ Professor A. Paul Alivisatos, Chair \\ Professor Peidong Yang \\ Professor Nitash P. Balsara
}

Spring 2007 
The dissertation of Haitao Liu is approved:

Chair _ Date

Date

Date

University of California, Berkeley

Spring 2007 


\title{
Chemistry of the Colloidal Group II-VI Nanocrystal Synthesis
}

\author{
Copyright 2007
}

by

Haitao Liu 


\begin{abstract}

\author{
by \\ Haitao Liu \\ Doctor of Philosophy in Chemistry \\ University of California, Berkeley \\ Professor A. Paul Alivisatos, Chair
}

Chemistry of the Colloidal Group II-VI Nanocrystal Synthesis

In the last two decades, the field of nanoscience and nanotechnology has witnessed tremendous advancement in the synthesis and application of group II-VI colloidal nanocrystals. The synthesis based on high temperature decomposition of organometallic precursors has become one of the most successful methods of making group II-VI colloidal nanocrystals. This method is first demonstrated by Bawendi and coworkers in 1993 to prepare cadmium chalcogenide colloidal quantum dots and later extended by others to prepare other group II-VI quantum dots as well as anisotropic shaped colloidal nanocrystals, such as nanorod and tetrapod. This dissertation focuses on the chemistry of this type of nanocrystal synthesis.

The synthesis of group II-VI nanocrystals was studied by characterizing the molecular structures of the precursors and products and following their time evolution in the synthesis. Based on these results, a mechanism was proposed to account for the 
reaction between the precursors that presumably produces monomer for the growth of nanocrystals. Theoretical study based on density functional theory calculations revealed the detailed free energy landscape of the precursor decomposition and monomer formation pathway. Based on the proposed reaction mechanism, a new synthetic method was designed that uses water as a novel reagent to control the diameter and the aspect ratio of CdSe and CdS nanorods.

Professor A. Paul Alivisatos

Dissertation Committee Chair 
To my father 


\section{Table of Contents}

Acknowledgements $\quad$ V

Abbreviations

Chapter 1 Introduction 1

1.1 Motivation 1

1.2 High Temperature Synthesis of Group II-VI Colloidal Nanocrystals in Organic

Solvent 2

1.3 Theoretical Modeling of Nanocrystalline Materials 6

1.4 Chemistry of the Nanocrystal Synthesis $\quad 7$

$\begin{array}{ll}\text { References } & 10\end{array}$

Chapter 2 Spectroscopic Characterization of the Precursors and Products 14

2.1 Introduction 14

$\begin{array}{ll}2.2 \text { Experimental } & 15\end{array}$

2.3 Characterization of Precursors 22

2.4 Characterization of Reaction Products 28

References 41

Chapter 3 Kinetics and Mechanism of the Synthesis of Group II-VI Nanocrystals 43

3.1 Introduction 43

3.2 Kinetics of Phosphine Chalcogenide Cleavage $\quad 44$

3.3 Mechanism of Precursor Decomposition and Monomer Formation 51 
Chapter 4 Density Functional Theory Study of the Precursor Evolution in the $\begin{array}{ll}\text { Synthesis of CdSe Nanocrystals } & 60\end{array}$

4.1 Introduction $\quad 60$

4.2 Theoretical Method 61

4.3 Results and Discussion $\quad 62$

$\begin{array}{ll}\text { 4.4 Conclusion } & 72\end{array}$

$\begin{array}{ll}\text { References } & 73\end{array}$

Chapter 5 Effect of Water on the Synthesis of CdSe and CdS Nanorods 75

$\begin{array}{ll}5.1 \text { Introduction } & 75\end{array}$

$\begin{array}{ll}5.2 \text { Experimental } & 77\end{array}$

$\begin{array}{ll}5.3 \text { Results and Discussion } & 79\end{array}$

5.4 Conclusions $\quad 86$

$\begin{array}{ll}\text { References } & 88\end{array}$

Chapter 6 Preparation of Asymmetric Nanostructures from CdTe Tetrapod $\begin{array}{ll}\text { Nanocrystals } & 90\end{array}$

$\begin{array}{ll}\text { 6.1 Introduction } & 90\end{array}$

6.2 Results and Discussions 93

$\begin{array}{ll}\text { References } & 103\end{array}$ 
Chapter 7 Conclusion and Outlook

7.1 Summary of Research

7.2 Outlook 


\section{Acknowledgements}

I am grateful to my advisor, Professor A. Paul Alivisatos, for his support and guidance during my years in graduate school. His attitude towards science and his openmindedness have greatly influenced my way of doing scientific research. I would like to acknowledge all the members of the Alivisatos group, especially to Dr. Jennifer Cha for guiding me through my first year of graduate school, Dr. Jonathan S. Owen, Steven Hughes, Dr. Andreu Cabot, Dr. Richard Robinson, Dr. Kimani A. Stancil, Dr. Haimei Zheng, Dr. Yue Wu, Dr. Gordana Dukovic, Alexander Mastroianni, Dr. Antonios G. Kanaras, Dr. Kevin M. Ryan, and Dr. Carsten Sönnichsen for various collaborations, Dr. Yadong Yin, Dr. Yi Cui, Dr. Emory Chan, Kristie Koski, Dr. Liberato Manna, Dr. Aihua $\mathrm{Fu}$, and Dr. Deborah Aruguete for sharing the laughs and optimisms. Thanks also to our administrative assistant, Rita Tidwell, for making the purchases faster and my life easier.

Outside Alivisatos group, I would like to thank Professor T. Andrew Taton for his kind support and guidance during my stay in his group before I moved to Berkeley, Dr. Tom Lowery for collaboration, Professor Robert G. Bergman and Professor T. Don Tilley for helpful discussion, and Dr. Herman van Halbeek for assistance on $2 \mathrm{D}^{31} \mathrm{P}$ NMR experiments. Financial support of this work was provided by the Director, Office of Science, Office of Basic Energy Sciences, Materials Sciences and Engineering Division, of the U.S. Department of Energy.

I am very grateful to my parents and my sister for their support. I thank my love, Lan Deng for her encouragement and help, both in research and in daily life. I would not have gone this far without them. 


\section{Abbreviations}

$\mathrm{CdO}$ : cadmium oxide; $\mathrm{Cd}(\mathrm{OA})_{2}$ : cadmium oleate; Cd-PA: cadmium alkyl-phosphonic acid complex; Cd-ODPA: cadmium $n$-octadecyl phosphonic acid complex; $\mathrm{H}_{2}$-ODPA: $n$ octadecyl phosphonic acid; $\mathrm{H}_{2}-\mathrm{OA}$ : oleic acid; $\mathrm{H}_{2}-\mathrm{PA}$ : alkyl-phosphonic acid; MS: mass spectrometry; MALDI: matrix assisted laser desorption/ionization; NMR: nuclear magnetic resonance; ODE: n-octadecene; PL: photoluminescence; SEM: scanning electron microscopy; TBP: tri- $n$-butyl phosphine; TBPO: tri- $n$-butyl phosphine oxide; TBPS: tri- $n$-butyl phosphine sulfide; TBPSe: tri- $n$-butyl phosphine selenide; TBPTe: trin-butyl phosphine telluride; $i$-TPPSe: tri-iso-propyl phosphine selenide; TEM: transmission electron microscopy; TOP: tri- $n$-octyl phosphine; TOPO: tri- $n$-octyl phosphine oxide; TOPS: tri- $n$-octyl phosphine sulfide; TOPSe: tri- $n$-octyl phosphine selenide; TOPTe: tri- $n$-octyl phosphine telluride; UV-Vis: Ultraviolet and visible absorption spectroscopy. 


\section{Chapter 1}

\section{Introduction}

\subsection{Motivation}

Colloidal nanocrystals are nanometer size objects composed of an inorganic core and a stabilizing organic shell. Usually, the inorganic core possesses useful properties while the organic shell renders the particle soluble and processable in common solvents. Group II-VI semiconductor nanocrystals have attracted a lot of attention in the last two decades. This class of nanocrystals has the general formula of $\mathrm{ME}$, where $\mathrm{M}=\mathrm{Zn}, \mathrm{Cd}$ and $\mathrm{E}=\mathrm{S}$, Se, Te. Due to the quantum size effect, group II-VI (as well as many other types of) semiconductor nanocrystals exhibit interesting size and shape dependent properties. One of the most cited examples is the size dependent band gap, especially when the size of the nanocrystal is comparable to the exciton Bohr radius of the core material. As a result of this size dependent band gap variation, the emission wavelength of spherical shaped CdSe nanocrystals can be easily tuned within the visible range by simply changing the diameter of the nanocrystal. Another important feature of the colloidal nanocrystals is their superior processability. Due to the presence of the organic shell, these nanocrystals can be processed from solution and easily incorporated into various organic matrixes. Especially, solution processing makes it possible to fabricate low cost electronic devices by spin coating. In a sense, colloidal semiconductor 
nanocrystals can be viewed as liquid semiconductors. Due to these unique properties, group II-VI colloidal nanocrystals have been used as the key pieces in the development of

novel fluorescent labels, ${ }^{1-3}$ light emitting diodes, ${ }^{4,5}$ solar cells, ${ }^{6-8}$ and other electronic devices. ${ }^{9}$

This dissertation focuses on the chemistry associated with the synthesis and postsynthesis modification of group II-VI nanocrystals. The organization of the dissertation is the following: In this chapter, I introduce the synthesis of group II-VI nanocrystals by thermo decomposition of precursors and the theoretical studies of nano-structured materials using ab intio calculations. In chapter 2, I present the characterization of the precursors and products of the synthesis of group II-VI nanocrystals. In chapter 3, I present the reaction kinetics of the nanocrystal syntheses and propose a reaction mechanism for the precursor-to-monomer conversion in the synthesis of group II-VI nanocrystals. In chapter 4, I use density functional theory calculations to map the overall energy landscape of the proposed reaction mechanism. Chapter 5 presents a new shape controlled synthesis of CdSe and CdS nanorods, which is designed based on the newly proposed reaction mechanism. In this synthesis, water was used as a reagent to control the diameter and aspect ratio of the nanorods. In chapter 6 , I demonstrate the asymmetric chemical modification of tetrapod shaped CdTe nanocrystals using Au nanocrystals. In chapter 7, I conclude my research and provide a brief outlook for future directions in the field.

\subsection{High Temperature Synthesis of Group II-VI Colloidal Nanocrystals in Organic}

\section{Solvent.}


The synthesis of group II-VI colloidal nanocrystals by thermal decomposition of precursors has been extensively used in the past decade. This synthetic approach was first reported for the synthesis of cadmium chalcogenide quantum dots, ${ }^{10}$ and later was extended to make nanorods, ${ }^{11,12}$ and tetrapods. ${ }^{12,13}$ The original synthesis is carried out by thermally decomposing a mixture of $\mathrm{CdMe}_{2}$ and tri-alkyl-phosphine chalcogenide in a hot TOPO solution. Later, a more synthetically convenient precursor, $\mathrm{CdO},{ }^{14,15}$ was introduced to replace $\mathrm{CdMe}_{2}$ and non-coordinating solvents, such as octadecene (ODE), were used to replace TOPO.$^{16}$ Besides its application in the synthesis of quantum dots, rods, and tetrapods of cadmium chalcogenides, this synthesis has also been extended to prepare $\mathrm{ZnS}$ and $\mathrm{ZnSe}$ nanocrystals ${ }^{17}$ as well as core-shell dots and rods, ${ }^{18,19}$ branched rods and tetrapods, ${ }^{20,21}$ and quantum dot - quantum well structures. ${ }^{22,23}$

The Bawendi-Murray synthesis of cadmium chalcogenide quantum dots. The first successful synthesis of high quality, almost defect free cadmium chalcogenide nanocrystals was reported by Bawendi and coworkers in $1993 .{ }^{10}$ In their synthesis, a cadmium precursor and a chalcogen precursor were co-injected into a mixture of TOPO and TOP at high temperature (usually $250^{\circ} \mathrm{C}-350^{\circ} \mathrm{C}$ ). Under these reaction conditions, the precursors decompose rapidly to produce nanocrystals that are capped by TOPO and TOP molecules in-situ. The unique feature of this synthesis is the use of high boiling point solvent (TOPO and TOP), which makes it possible to carry out the reaction at much higher temperature than any previous methods. Presumably, the high temperature anneals the inorganic lattice to produce high quality, almost defect free nanocrystals. By controlling the reaction conditions, such as temperature and time, nanocrystals of different sizes could be routinely obtained with less than $10 \%$ standard deviation of the 
diameter. This synthetic method has tremendous impact on the research of group II-VI nanocrystals. As time of writing (March, 2007), the original report on the synthesis of cadmium chalcogenide quantum dot has been cited for more than 2000 times.

The precursors used in the original Bawendi-Murray synthesis are organic or organometallic compounds such as $\mathrm{CdMe}_{2}$, tri-n-octylphosphine chalcogenides and bistrimethylsily chalcogenides. The success of this synthetic method triggered the high temperature synthesis of other group II-VI, group III-V, and group IV-VI nanocrystals. Some notable examples can be found in table 1-1.

\begin{tabular}{cccc}
\hline Material & Cation Precursor & Anion Precursor & Ref \\
\hline $\mathrm{ZnS} / \mathrm{ZnSe}$ & $\mathrm{ZnEt}_{2}$ & TOPSe & 24 \\
$\mathrm{GaAs} / \mathrm{InAs} / \mathrm{InP}$ & $\mathrm{GaCl}_{3} / \mathrm{InCl}_{3}$ & $\mathrm{As}(\mathrm{TMS})_{3} / \mathrm{P}(\mathrm{TMS})_{3}$ & $25-27$ \\
$\mathrm{PbS} / \mathrm{Se} / \mathrm{Te}$ & $\mathrm{Pb}(\mathrm{Ac})_{2}$ & $\mathrm{TOPS} / \mathrm{TOPSe} / \mathrm{TOPTe}$ & $28-30$ \\
\hline
\end{tabular}

Table 1-1. High temperature solution phase synthesis of semiconductor nanocrystals

Shape controlled synthesis of group II-VI nanocrystals. The original BawendiMurray method only produces spherical shaped nanocrystals. Later, it was discovered by Alivisatos and coworkers that when alkyl-phosphonic acid was added to the growth medium and high concentration of precursors was used, rod shaped CdSe nanocrystals, or nanorod, could be produced. ${ }^{11,12}$ It was rationalized that phosphonic acid adsorbs to the side facets (e.g., 11-20) of the nanorod much stronger than to the end facets (e.g., 0002 and 000-2). It was believed that adsorption of alkyl-phosphonic acid molecules modulates 
the growth rate of different crystal facets. Since the end facets are relatively less covered by alkyl-phosphonic acid than the side facets are, growth of the end facets are favored relative the side facets, resulting in rod shaped nanocrystals. By controlling the reaction conditions, other anisotropic shaped nanocrystals were also synthesized, such as tetrapods ${ }^{13}$ and hyper-branched ${ }^{21}$ nanocrystals.

The use of metal oxide and non-coordinating solvents. The original BawendiMurray method is very successful in producing high quality nanocrystals. However, it still has several drawbacks. Especially, the precursors and solvents used in the synthesis, especially $\mathrm{CdMe}_{2}$, bis-trimethylsily chalcogenides, and TOP, are either highly toxic or pyrophoric. To address this problem, Peng and coworkers have reported a modified synthesis of cadmium chalcogenides using $\mathrm{CdO}$ as the cadmium precursor. ${ }^{14}$ In this synthesis, CdO was first dissolved in alkyl-phosphonic acid or carboxylic acid at high temperature and phosphine chalcogenide was then injected to grow nanocrystals. The same group also used non-coordinating solvents, such as ODE, to replace TOPO and TOP to make a variety of nanocrystals, including $\mathrm{Cd}$ and $\mathrm{Zn}$ chalcogenides. ${ }^{16,17}$

Other synthetic methods. Though numerous other variations of precursors, solvent, and surfactants have been studied in the synthesis of group II-VI nanocrystals, these methods were not as widely used as the ones discussed above. From the chemist point of view, the use of elemental chalcogene instead of phosphine chalcogenide represents maybe another major modification of the original Bawendi-Murray synthesis. $^{16,31}$

Size and shape evolution of group II-VI nanocrystals during the growth. A number of publications have documented the temporal evolution of nanocrystal size and 
shape during the growth of group II-VI nanocrystals, especially for the case of CdSe. Alivisatos and coworkers have studied the time evolution of CdSe quantum dot in TOPO ${ }^{26}$ They suggested that the growth of nanocrystal is a diffusion limited process. They also find that the size dispersion of nanocrystal decreases at high 'monomer' concentration, a phenomenon they called "focusing of size distribution". Another study by Muvalley and coworkers documented the growth of CdSe quantum dot in a noncoordinating solvent using oleic acid as the surfactant. ${ }^{32}$ They observed that the concentration of nanocrystal did not change appreciably during the whole growth period. This observation suggests that nanocrystals nucleate only at the very beginning of the reaction which is followed by growth of existing nuclei. Alivisatos group and Peng group have studied the shape evolution of CdSe nanorod in TOPO prepared from $\mathrm{CdMe}_{2}$ and CdO, respectively. ${ }^{12,33,34}$ They have found that high concentration of precursors is essential in producing anisotropic shaped nanocrystals. The growth of more exotic shaped materials, such as tetrapod, has also been studied, but to a less extend. ${ }^{13}$

\subsection{Theoretical Modeling of Nanocrystalline Materials}

$a b$ initio calculation has played a significant role in understanding the physics and chemistry of nanocrystalline materials. Due to the large size of the nanocrystal, much of the related work has been based on density functional theory (DFT) method, which provides a good balance between accuracy and speed.

The ground state geometry and electronic structure of group II-VI clusters have

been calculated by several groups. ${ }^{35-38}$ For the case of CdSe, the cohesive energy per CdSe unit generally increases with increasing size of the cluster. However, it was also 
found that certain clusters are much more stable than their neighbors. These clusters are often called 'magic clusters'. Several pieces of experimental evidence suggest that this kind of cluster exist in the synthesis and are likely to play an important role in the nucleation and growth of nanocrystals. ${ }^{34,36}$

The binding of surfactant molecule to the nanocrystal surface plays an important role in the growth of nanocrystal and has been subject to a number of studies. ${ }^{37,39,40}$ For the case of $\mathrm{CdSe}$, it is now generally agreed that surfactant molecules bind to electron deficient surface $\mathrm{Cd}$ atoms via the electron rich oxygen, nitrogen, or phosphorous atoms. The binding energy ranges from $0.5-1.0 \mathrm{eV}$ depending on the nature of the surfactant. These studies also show that the binding energy is different for different crystal facets, which provides an explanation for the formation of nanorod in the presence of certain surfactant molecules, such as alkyl-phosphonic acid.

\subsection{Chemistry of the Nanocrystal Synthesis}

The synthesis of group II-VI nanocrystals has been studied for more than a decade. Current state-of-the-art synthetic methods routinely produce high quality nanocrystalline materials with desired size and shape. However, from the chemist point of view, the understanding of these syntheses is still at a very rudimentary stage. With the exception of inorganic nanocrystals, the precursors and products of the synthesis have not been characterized at all. As a consequence, little is known about the detailed reactions between the precursors. In a similar vein, the nanocrystal nucleation and growth process have not been understood in terms of their associated chemistry. 
Current understanding of the nanocrystal synthesis offers little guidance on the selection of reaction conditions and precursors. As a result, synthetic work in the field was routinely conducted in a trial-and-error fashion. The synthesis of each type of material must be developed via tedious empirical optimizations, which requires extensive search of a large parameter space, including precursor, solvent, surfactant, and reaction conditions.

In addition to its obvious scientific contribution, understanding the chemistry associated with the nanocrystal synthesis could benefit the nanoscience community in a number of practical ways. First, it will offer guidance in searching parameter space when a new material is to be prepared. Second, it will allow rational control of nanocrystal size and shape and offers the possibility of synthesizing new exotic shaped nanocrystals. Third and most importantly, it will open the door to multi-step synthesis of complex nanostructures, much like the way organic chemists have been doing when synthesizing complex organic molecules, such as natural products.

Precursor vs. monomer. In this dissertation, precursor refers to the starting material used to prepare the nanocrystals while monomer refers to the inorganic unit immediately available for the nucleation and growth of nanocrystal. Precursors are stable compounds that can be well characterized by common analytical techniques such as NMR, MS, and elemental analysis. Common precursors used in the synthesis of group IIVI nanocrystals are phosphine chalcogenides, $\mathrm{CdMe}_{2}$, Cd-ODPA complex, and Cd-OA complex. Monomer, on the other hand, is an unstable intermediate between precursor and nanocrystal and is much harder to characterize than the precursors. So far, there has been no report on the structure of the monomer. Monomer is produced by the reaction between 
precursors and is consumed by the nanocrystal nucleation and growth process. As a result, the monomer concentration is dynamically changing during the synthesis of nanocrystals. The concentration of monomer controls the nucleation and growth of nanocrystal: if the monomer concentration is higher than the nucleation threshold, then new nuclei of nanocrystal are produced while existing nanocrystals grow; if the monomer concentration is higher than the solubility of the nanocrystal but lower than the nucleation threshold, then no nucleation occurs and only the existing nanocrystals grow; if the monomer concentration is lower than the solubility of nanocrystal, then the nanocrystals dissolve. ${ }^{34}$

In the literature, precursor and monomer are often, regrettably, not distinguished in the discussion of nanocrystal nucleation and growth. ${ }^{12}$ This confusion is in part due to the limited understanding of the chemical process of nanocrystal synthesis. A clear distinction between these two terms is made in this dissertation. In fact, one of major goals of this dissertation is to understand the kinetics and mechanism of monomer generation from the precursors. 


\section{References}

(1) Bruchez, M.; Moronne, M.; Gin, P.; Weiss, S.; Alivisatos, A. P. Science 1998, 281, 2013-2016.

(2) Alivisatos, P. Nature Biotech. 2004, 22, 47-52.

(3) Gerion, D.; Parak, W. J.; Williams, S. C.; Zanchet, D.; Micheel, C. M.; Alivisatos, A. P. J. Am. Chem. Soc. 2002, 124, 7070-7074.

(4) Colvin, V. L.; Schlamp, M. C.; Alivisatos, A. P. Nature 1994, 370, 354357.

(5) Coe, S.; Woo, W. K.; Bawendi, M.; Bulovic, V. Nature 2002, 420, 800803.

(6) Huynh, W. U.; Peng, X. G.; Alivisatos, A. P. Adv. Mater. 1999, 11, 923927.

(7) Huynh, W. U.; Dittmer, J. J.; Alivisatos, A. P. Science 2002, 295, $2425-$ 2427.

(8) Gur, I.; Fromer, N. A.; Geier, M. L.; Alivisatos, A. P. Science 2005, 310, $462-465$.

(9) Cui, Y.; Banin, U.; Bjork, M. T.; Alivisatos, A. P. Nano Lett. 2005, 5, $1519-1523$.

(10) Murray, C. B.; Norris, D. J.; Bawendi, M. G. J. Am. Chem. Soc. 1993, 115, $8706-8715$

(11) Peng, X. G.; Manna, L.; Yang, W. D.; Wickham, J.; Scher, E.; Kadavanich, A.; Alivisatos, A. P. Nature 2000, 404, 59-61. 
(12) Manna, L.; Scher, E. C.; Alivisatos, A. P. J. Am. Chem. Soc. 2000, 122, 12700-12706.

(13) Manna, L.; Milliron, D. J.; Meisel, A.; Scher, E. C.; Alivisatos, A. P. Nature Mater. 2003, 2, 382-385.

(14) Peng, Z. A.; Peng, X. G. J. Am. Chem. Soc. 2001, 123, 183-184.

(15) Qu, L. H.; Peng, Z. A.; Peng, X. G. Nano Lett. 2001, 1, 333-337.

(16) Yu, W. W.; Peng, X. G. Angew. Chem. Int. Ed. 2002, 41, 2368-2371.

(17) Lin, S. L.; Pradhan, N.; Wang, Y. J.; Peng, X. G. Nano Lett. 2004, 4, 2261-2264.

(18) Manna, L.; Scher, E. C.; Li, L. S.; Alivisatos, A. P. J. Am. Chem. Soc. 2002, 124, 7136-7145.

(19) Peng, X. G.; Schlamp, M. C.; Kadavanich, A. V.; Alivisatos, A. P. J. Am. Chem. Soc. 1997, 119, 7019-7029.

(20) Milliron, D. J.; Hughes, S. M.; Cui, Y.; Manna, L.; Li, J. B.; Wang, L. W.; Alivisatos, A. P. Nature 2004, 430, 190-195.

(21) Kanaras, A. G.; Sonnichsen, C.; Liu, H. T.; Alivisatos, A. P. Nano Lett. 2005, 5, 2164-2167.

(22) Battaglia, D.; Li, J. J.; Wang, Y. J.; Peng, X. G. Angew. Chem. Int. Ed. 2003, 42, 5035-5039.

(23) Battaglia, D.; Blackman, B.; Peng, X. G. J. Am. Chem. Soc. 2005, 127, 10889-10897.

(24) Hines, M. A.; Guyot-Sionnest, P. J. Phys. Chem. B 1998, 102, 3655-3657. 
(25) Guzelian, A. A.; Katari, J. E. B.; Kadavanich, A. V.; Banin, U.; Hamad, K.; Juban, E.; Alivisatos, A. P.; Wolters, R. H.; Arnold, C. C.; Heath, J. R. Journal of Physical Chemistry 1996, 100, 7212-7219.

(26) Peng, X. G.; Wickham, J.; Alivisatos, A. P. J. Am. Chem. Soc. 1998, 120, 5343-5344.

(27) Yun-Wei Cao, U. B. Angewandte Chemie International Edition 1999, 38, 3692-3694.

(28) Houtepen, A. J.; Koole, R.; Vanmaekelbergh, D.; Meeldijk, J.; Hickey, S. G. J. Am. Chem. Soc. 2006, 128, 6792-6793.

(29) Murphy, J. E.; Beard, M. C.; Norman, A. G.; Ahrenkiel, S. P.; Johnson, J. C.; Yu, P.; Micic, O. I.; Ellingson, R. J.; Nozik, A. J. J. Am. Chem. Soc. 2006, 128, 32413247.

(30) Lifshitz, E.; Brumer, M.; Kigel, A.; Sashchiuk, A.; Bashouti, M.; Sirota, M.; Galun, E.; Burshtein, Z.; LeQuang, A. Q.; Ledoux-Rak, I.; Zyss, J. J. Phys. Chem. B 2006, $110,25356-25365$.

(31) Jasieniak, J.; Bullen, C.; vanEmbden, J.; Mulvaney, P. J. Phys. Chem. B 2005, 109, 20665-20668.

(32) Bullen, C. R.; Mulvaney, P. Nano Lett. 2004, 4, 2303-2307.

(33) Peng, Z. A.; Peng, X. G. J. Am. Chem. Soc. 2001, 123, 1389-1395.

(34) Peng, Z. A.; Peng, X. G. J. Am. Chem. Soc. 2002, 124, 3343-3353.

(35) Deglmann, P.; Ahlrichs, R.; Tsereteli, K. J. Chem. Phys. 2002, 116, $1585-$ 1597. 
(36) Yu, M.; Fernando, G. W.; Li, R.; Papadimitrakopoulos, F.; Shi, N.; Ramprasad, R. App. Phys. Lett. 2006, 88, 231910.

(37) Puzder, A.; Williamson, A. J.; Zaitseva, N.; Galli, G.; Manna, L.; Alivisatos, A. P. Nano Lett. 2004, 4, 2361-2365.

(38) Puzder, A.; Williamson, A. J.; Gygi, F.; Galli, G. Phys. Rev. Lett. 2004, 92, 217401.

(39) Rempel, J. Y.; Trout, B. L.; Bawendi, M. G.; Jensen, K. F. J. Phys. Chem. B 2005, 109, 19320-19328.

(40) Manna, L.; Wang, L. W.; Cingolani, R.; Alivisatos, A. P. J. Phys. Chem. B 2005, 109, 6183-6192. 


\section{Chapter 2}

\section{Spectroscopic Characterization of the}

\section{Precursors and Products}

Reproduced in part with permission from "Liu, H.; Owen, J. S.; Alivisatos, A. P. "Mechanistic Study of Precursor Evolution in Colloidal Group II-VI Semiconductor Nanocrystal Synthesis" J. Am. Chem. Soc. 2007, 129, 305-312, Copyright 2007 American Chemical Society.

\subsection{Introduction}

Even though the synthesis of group II-VI nanocrystals can now be routinely carried out to produce high quality nanocrystals, the chemistry associated with the formation of this type of nanocrystals has not been well understood. Previous mechanistic study on the synthesis of group II-VI nanocrystals has been exclusively focused on following the kinetics of nanocrystal growth and shape evolution by monitoring the size and shape of nanocrystals using UV-Vis, PL, and TEM. These studies have generated many important concepts such as size distribution focusing, ${ }^{1}$ selective adhesion shape control, $^{2-4}$ and branching, ${ }^{3,5,6}$. However, one of the most fundamental questions remains unsolved: how precursors are converted into inorganic materials. 
As the first step toward understanding the mechanism of nanocrystal formation on the molecular level, I present an extensive characterization of the reaction precursors and products of the synthesis of group II-VI nanocrystals. Using a combination of NMR $\left({ }^{1} \mathrm{H}\right.$,

${ }^{13} \mathrm{C}$, and ${ }^{31} \mathrm{P}$ ) and mass spectroscopy I have investigated the synthesis of group II-VI semiconductor nanocrystals in TOPO and ODE by following the disappearance and appearance of molecular precursors and products. This is the first time that this complex reaction was fully characterized at the molecular level.

\subsection{Experimental}

Tri-n-octylphosphine (TOP, 97\%, Strem), tri- $n$-butylphosphine (TBP, 99\%, Strem), tri-iso-propylphosphine (Aldrich), TOPO (Aldrich, 99\%, lot number $24801 \mathrm{MB}$, and 04017PC). n-octadecylphosphonic acid ( $\mathrm{H}_{2}-\mathrm{ODPA}$, Polycarbon), $\mathrm{CdO}$ (Aldrich, 99.99+\%), ZnO (Aldrich, 99.99+\%), oleic acid (H-OA, Aldrich, 99\%), n-nonane-d $\mathrm{d}_{20}$ (Aldrich, 98 atom \%D), $n$-decane-d $\mathrm{d}_{22}($ Arcos, 99 atom \%D), ODE (Aldrich, 90\%) were used as received. Standard air sensitive techniques were used to handle air and moisture sensitive compounds.

NMR methods. All NMR $\left({ }^{1} \mathrm{H},{ }^{13} \mathrm{C}\right.$, and $\left.{ }^{31} \mathrm{P}\right)$ spectra were collected on a $400 \mathrm{MHz}$ Bruker Advance spectrometer. ${ }^{31} \mathrm{P}$ NMR spectra were acquired either without proton decoupling or with inverse gated decoupling and care was taken to ensure adequate relaxation $\left(\geq 5 \times \mathrm{T}_{1}\right)$ between pulses. In situ experiments were conducted under vacuum in flame sealed NMR tubes. These samples were inserted into a preheated NMR probe that was calibrated using ethylene glycol as a standard according to an established procedure. ${ }^{7} \mathrm{CDCl}_{3}$ solutions of aliquots from the TOPO based reaction were prepared in 
air. Control experiments showed that $\mathrm{CDCl}_{3}$ solutions of TOPE and TBPE $(\mathrm{E}=\mathrm{Se}, \mathrm{S})$ stored in air at room temperature are air stable for several weeks, with only $<2 \%$ conversion to TOPO by ${ }^{31} \mathrm{P}$ NMR spectroscopy.

The concentration of phosphine chalcogenide was obtained by comparing the integral of its ${ }^{31} \mathrm{P}$ NMR peak with that of the phosphine oxide peak and assuming the total concentration of the two species was constant during the reaction. This assumption was verified to be valid ( $< \pm 2 \%$ error) by using an internal standard (ethylphosphonic acid diethyl ester) in the in situ experiments. The ${ }^{31} \mathrm{P}$ NMR resonances of TOPO and TOPS partially overlap, in which case peak integrals were obtained by deconvolution of the two resonances using MestReC (Mestrelab Research).

Synthesis and characterization of TOPSe, tri-n-octylphosphine sulfide (TOPS), tri-n-butylphosphine sulfide (TBPS), TBPSe, tri-n-butylphosphine telluride (TBPTe). Traditional syntheses of group II-VI nanocrystals use a mixture of TOP and TOPE (or mixture of TBP and TBPE) as the injection solution. ${ }^{8}$ To simplify the analysis, we used the pure phosphine chalcogenide instead of a mixture with its parent phosphine. Phosphine chalcogenides were prepared by stirring the appropriate phosphine with a stoichiometric or excess amount of elemental $\mathrm{S} / \mathrm{Se} / \mathrm{Te}$ in a glove box at room temperature. The supernatant was separated from the excess solid chalcogen and was found to be pure by NMR $\left({ }^{1} \mathrm{H},{ }^{13} \mathrm{C}\right.$, and $\left.{ }^{31} \mathrm{P}\right)$ and elemental analysis.

TBPS: A mixture of TBP (2.02 g, $0.010 \mathrm{~mol})$ and sulfur $(0.435 \mathrm{~g}, 0.014 \mathrm{~mol})$ was stirred at r.t. in a glove box overnight. The supernatant was used in the experiments without further purification. ${ }^{1} \mathrm{H}\left(\mathrm{CDCl}_{3}, \delta \mathrm{ppm}\right)$ : 1.63-1.70 (m, 6H), 1.37-1.47 (m, 6H), 1.23-1.32 (m, 6H), $0.79(\mathrm{t}, 9 \mathrm{H}, J=7.3 \mathrm{~Hz}) \cdot{ }^{13} \mathrm{C}\left(\mathrm{CDCl}_{3}, \delta \mathrm{ppm}\right.$, more than 4 peaks 
appeared due to the coupling between ${ }^{13} \mathrm{C}$ and ${ }^{31} \mathrm{P}$ ): $30.49,29.99,24.11,24.07,23.69$, 23.54, 13.31. ${ }^{31} \mathrm{P}\left(\mathrm{CDCl}_{3}, \delta \mathrm{ppm}\right)$ : 48.7. Anal. (calcd, found): $\mathrm{C}(61.49,61.11), \mathrm{H}$ (11.61, 11.84). TBPSe, TBPTe, TOPSe, and TOPS were prepared similarly with longer reaction times.

TBPSe: ${ }^{1} \mathrm{H}\left(\mathrm{CDCl}_{3}, \delta\right.$ ppm): 1.73-1.79 (m, 6H), 1.36-1.46 (m, 6H), 1.23-1.33 (m, $6 \mathrm{H}), 0.79(\mathrm{t}, 9 \mathrm{H}, J=7.3 \mathrm{~Hz}) .{ }^{31} \mathrm{P}\left(\mathrm{CDCl}_{3}, \delta \mathrm{ppm}\right): 36.8, J_{\mathrm{P}-\mathrm{Se}}=680 \mathrm{~Hz}$. Anal. (calcd, found): C (51.24, 51.37), H (9.68, 9.78).

TBPTe: The mixture solidified into a yellow crystalline solid after it was stored in a glove box at r.t. for 30 days. ${ }^{1} \mathrm{H}\left(\mathrm{CDCl}_{3}, \delta \mathrm{ppm}\right): 1.96-2.03(\mathrm{~m}, 6 \mathrm{H}), 1.38-1.56(\mathrm{~m}, 12$ $\mathrm{H}), 0.93(\mathrm{t}, 9 \mathrm{H}, J=7.2 \mathrm{~Hz}) .{ }^{13} \mathrm{C}\left(\mathrm{CDCl}_{3}, \delta \mathrm{ppm}\right): 30.45,30.09,26.56,26.52,23.55,23.40$, 13.61. ${ }^{31} \mathrm{P}\left(\mathrm{CDCl}_{3}, \delta \mathrm{ppm}\right):-13.2 . J_{\mathrm{P}-\mathrm{Te}}\left({ }^{125} \mathrm{Te}\right)=1655 \mathrm{~Hz}, J_{\mathrm{P}-\mathrm{Te}}\left({ }^{123} \mathrm{Te}\right)=1370 \mathrm{~Hz}$.

TOPSe: ${ }^{1} \mathrm{H}\left(\mathrm{CDCl}_{3}, \delta\right.$ ppm): 1.83-1.90 (m, 6H), 1.49-1.59 (m, 6H), 1.32-1.39 (m, $6 \mathrm{H}), 1.22-1.29(\mathrm{~m} \mathrm{24H}), 0.84(\mathrm{t}, 9 \mathrm{H}, J=6.8 \mathrm{~Hz}) \cdot{ }^{31} \mathrm{P}\left(\mathrm{CDCl}_{3}, \delta \mathrm{ppm}\right): 36.4 . J_{\mathrm{P}-\mathrm{Se}}=678$ Hz. ${ }^{77} \mathrm{Se}\left(\mathrm{CDCl}_{3}, \delta \mathrm{ppm}\right):-377,-386$. Anal. (calcd, found): C (64.11, 64.46), H(11.3, 11.65).

TOPS: ${ }^{1} \mathrm{H}\left(\mathrm{CDCl}_{3}, \delta\right.$ ppm). 1.72-1.79 (m, 6H), 1.49-1.59 (m, 6H), 1.32-1.37 (m, $6 \mathrm{H}), 1.21-1.32(\mathrm{~m}, 24 \mathrm{H}), 0.85(\mathrm{t}, 9 \mathrm{H}, J=6.6 \mathrm{~Hz}) .{ }^{31} \mathrm{P}\left(\mathrm{CDCl}_{3}, \delta \mathrm{ppm}\right)$ : 48.6. Anal. (calcd, found): $\mathrm{C}(71.58,72.05), \mathrm{H}(12.77,13.06)$.

Synthesis of cadmium and zinc oleic acid complexes (M-OA, $\mathrm{M}=\mathbf{Z n}, \mathbf{C d}){ }^{9}$ Cd-OA: To a $25 \mathrm{~mL}$ flask was added H-OA (4.55 g, $16 \mathrm{mmol})$ and $\mathrm{CdO}(0.518 \mathrm{~g}, 4.0$ mmol). The mixture was degassed at $100{ }^{\circ} \mathrm{C}, 250 \mathrm{mtorr}$ for $30 \mathrm{~min}$. The flask was then filled with $\mathrm{Ar}$ and heated to $190{ }^{\circ} \mathrm{C}$ to dissolve $\mathrm{CdO}$. After the dissolution of $\mathrm{CdO}$, the mixture was cooled to $110{ }^{\circ} \mathrm{C}$ and degassed again at 300 mtorr for $20 \mathrm{~min}$. The solution 
was then cooled to r.t. and stored in a freezer under $\mathrm{N}_{2}$. A stock solution was prepared by dissolving $2.40 \mathrm{~g}$ of this complex in $1.60 \mathrm{~g}$ of $n$-nonane- $\mathrm{d}_{20}$ and was used in the synthesis of $\mathrm{CdS}, \mathrm{CdSe}$, and CdTe nanocrystals. Zn-OA was prepared similarly by dissolving ZnO $(0.167 \mathrm{~g}, 2.05 \mathrm{mmol})$ in a mixture of H-OA $(2.319 \mathrm{~g}, 8.02 \mathrm{mmol})$ and ODE $(2.50 \mathrm{~g})$ at $300^{\circ} \mathrm{C}$ under Ar followed by degassing at $100{ }^{\circ} \mathrm{C}$. The neat reaction mixture was used in the synthesis of $\mathrm{ZnS}, \mathrm{ZnSe}$, and $\mathrm{ZnTe}$ nanocrystals.

\section{Synthesis of CdSe nanocrystal in TOPO/Hz-ODPA at $260{ }^{\circ} \mathrm{C}$ - the 'doubly}

degassed' protocol. To a $25 \mathrm{~mL}$ three-neck flask equipped with a condenser and a thermocouple adapter was added TOPO (2.73 g, $7.06 \mathrm{mmol}), \mathrm{H}_{2}$-ODPA (1.07 g, 3.20 $\mathrm{mmol})$, and $\mathrm{CdO}(0.204 \mathrm{~g}, 1.60 \mathrm{mmol})$. The mixture was degassed at $120{ }^{\circ} \mathrm{C}$ and 200 400 mtorr pressure for $60 \mathrm{~min}$. The flask was then filled with Ar and the temperature was raised to $320^{\circ} \mathrm{C}$ to dissolve $\mathrm{CdO}$. After dissolving $\mathrm{CdO}$, the temperature was lowered to $150-180{ }^{\circ} \mathrm{C}$ and the pressure was reduced to $\sim 300$ mtorr for $60 \mathrm{~min}$ (this step will be referred to in the text as the 'second degassing'). The flask was then filled with Ar and the temperature was raised to $270{ }^{\circ} \mathrm{C}$. TOPSe $(0.70 \mathrm{~g}, 1.6 \mathrm{mmol})$ was injected and the temperature was allowed to stabilize at $260 \pm 2{ }^{\circ} \mathrm{C}$. The amount of TOPSe injected $(1.4 \pm$ $0.1 \mathrm{mmol}$ ) was measured as the difference between the mass of the syringe before and after the injection. Aliquots taken after the injection of TOPSe were dissolved in $\mathrm{CDCl}_{3}$ and transferred to NMR tubes in air. NMR spectra of the aliquots were collected within $24 \mathrm{hr}$ of sampling.

In situ monitoring of the synthesis of CdSe nanocrystal in $\boldsymbol{n}$-nonane-d $\mathbf{2}_{20}$. To a $5 \mathrm{~mm}$ NMR tube was added TOPSe $(0.0809 \mathrm{~g}, 0.18 \mathrm{mmol})$, the Cd-OA stock solution (0.375 g, $0.18 \mathrm{mmol}$ of $\mathrm{Cd}^{2+}$ and $0.72 \mathrm{mmol}$ of H-OA/OA), and $n$-nonane-d $\mathrm{d}_{20}(0.0478 \mathrm{~g})$. 
The mixture was degassed by four cycles of freeze-pump-thaw before the NMR tube was flame sealed under vacuum. The NMR probe was then pre-heated to the desired reaction temperature and the sample was inserted into the probe and allowed to temperature equilibrate for $4 \mathrm{~min}$ before NMR spectra $\left({ }^{1} \mathrm{H},{ }^{13} \mathrm{C}\right.$, and $\left.{ }^{31} \mathrm{P}\right)$ were collected. NMR spectra were also collected at room temperature before and after the reaction to analyze the reaction products.

Synthesis of ME (M=Cd, Zn; $E=S$, Se, Te) nanocrystals in non-coordinating solvent in sealed NMR tube. CdS: In a $5 \mathrm{~mm}$ NMR tube was added Cd-OA stock solution (0.14 g, $0.060 \mathrm{mmol} \mathrm{Cd})$, TBPS (16 mg, $0.068 \mathrm{mmol})$, and $n$-decane- $\mathrm{d}_{22}(0.30 \mathrm{~g})$. The mixture was degassed by three cycles of freeze-pump-thaw before it was flame sealed under reduced pressure (300 mtorr). The mixture was heated to $150{ }^{\circ} \mathrm{C}$ for $2.5 \mathrm{~h}$. ${ }^{31} \mathrm{P}$ NMR $\left(\mathrm{C}_{9} \mathrm{D}_{20} / \mathrm{C}_{10} \mathrm{D}_{22}, \delta \mathrm{ppm}\right): 57.0$ (TBPO, identified by addition of TBPO), 50.7 (TBPS). Selected $(\mathrm{OA})_{2} \mathrm{O}$ peaks in the ${ }^{13} \mathrm{C}$ NMR spectrum $\left(\mathrm{C}_{9} \mathrm{D}_{20} / \mathrm{C}_{10} \mathrm{D}_{22}, \delta \mathrm{ppm}\right): 168.5$, 130.1, 129.7, and 35.4.

CdTe: This is similar to the synthesis of CdS nanocrystal in non-coordinating solvent using TBPTe. The mixture was heated to $120{ }^{\circ} \mathrm{C}$ in an oil bath for $20 \mathrm{~min} .{ }^{31} \mathrm{P}$ NMR $\left(\mathrm{C}_{9} \mathrm{D}_{20} / \mathrm{C}_{10} \mathrm{D}_{22}, \delta\right.$ ppm): $52.9 \mathrm{ppm}$ (TBPO). Selected $(\mathrm{OA})_{2} \mathrm{O}$ peaks in the ${ }^{13} \mathrm{C}$ NMR spectrum $\left(\mathrm{C}_{9} \mathrm{D}_{20} / \mathrm{C}_{10} \mathrm{D}_{22}, \delta \mathrm{ppm}\right): 168.7,130.1,129.8,35.4$.

ZnS: This is similar to the synthesis of CdSe nanocrystal in non-coordinating solvent using TBPS (62 mg, $0.26 \mathrm{mmol})$ and Zn-OA stock solution $(0.64 \mathrm{~g}, 0.25 \mathrm{mmol}$ $\mathrm{Zn}$ ) in ODE solvent. The mixture was heated to $265{ }^{\circ} \mathrm{C}$ in an oil bath for $42 \mathrm{~min} .{ }^{31} \mathrm{P}$ NMR (crude reaction mixture, without ${ }^{2} \mathrm{H}$ lock, $\delta$ ppm): 56.6 (TBPO), 46.3 (TBPS). 
Selected $(\mathrm{OA})_{2} \mathrm{O}$ peaks in the ${ }^{13} \mathrm{C}$ NMR spectrum (crude reaction mixture, without ${ }^{2} \mathrm{H}$ lock, $\delta$ ppm): 167.9, 129.3, 129.0, 34.6.

ZnSe: This is similar to the synthesis of CdSe nanocrystal in non-coordinating solvent using TBPSe $(77 \mathrm{mg}, 0.27 \mathrm{mmol})$ and $\mathrm{Zn}-\mathrm{OA}$ stock solution $(0.64 \mathrm{~g}, 0.25 \mathrm{mmol}$ $\mathrm{Zn}$ ) in ODE solvent. The mixture was heated to $265{ }^{\circ} \mathrm{C}$ in an oil bath for $42 \mathrm{~min} .{ }^{31} \mathrm{P}$ NMR (without ${ }^{2} \mathrm{H}$ lock, $\delta$ ppm): $53.6 \mathrm{ppm}$ (TBPO), 35.0 (TBPSe). Selected (OA) $)_{2} \mathrm{O}$ peaks in the ${ }^{13} \mathrm{C}$ NMR spectrum (without ${ }^{2} \mathrm{H}$ lock, $\delta$ ppm): 167.9, 129.3, 129.0, 34.6.

ZnTe: This is similar to the synthesis of CdSe nanocrystal in non-coordinating solvent using TBPTe $(0.12 \mathrm{~g}, 0.36 \mathrm{mmol})$, TBP $(0.12 \mathrm{~g}, 0.59 \mathrm{mmol})$, Zn-OA stock solution $(0.82 \mathrm{~g}, 0.32 \mathrm{mmol} \mathrm{Zn})$, and $n$-decane- $\mathrm{d}_{22}(0.39 \mathrm{~g})$. The mixture was heated to $230{ }^{\circ} \mathrm{C}$ in an oil bath for $150 \mathrm{~min} .{ }^{31} \mathrm{P}$ NMR ( $\left.\delta \mathrm{ppm}\right): 53.5 \mathrm{ppm}$ (TBPO), -25.0 (TBPTe and TBP, due to fast exchange of Te between TBPTe and TBP).

Karl-Fischer titration. The moisture content of reaction mixture and pure reagents was determined by volumetric Karl-Fischer titration using HYDRANAL reagents (Aldrich). Briefly, $1.0 \mathrm{~g}$ of the sample to be titrated was mixed with $5.0 \mathrm{~g}$ of HYDRANAL-Solvent 2E and titrated by dropwise addition of HYDRANAL-Titrant 2E via a syringe in a glove box. The titration was stopped when the solution turns into a yellow-brown color, indicating the presence of unreacted titrant. A control experiment was carried out to determine the moisture content in the HYDRANAL-Solvent 2E which was deducted as a background from all the titration results.

Titration of water produced during the dissolution of $\mathrm{CdO}$ in $\mathrm{H}_{2}$ ODPA/TOPO. To a $30 \mathrm{~mL}$ vial in a glove box was added TOPO (1.0 g), $\mathrm{H}_{2}$-ODPA (1.07 $\mathrm{g}, 3.2 \mathrm{mmol})$, and $\mathrm{CdO}(0.212 \mathrm{~g}, 1.6 \mathrm{mmol})$. The vial was tightly capped and brought out 
of the glove box before it was heated to high temperature to dissolve CdO. The vial was then brought into the glove box and the mixture was titrated using the method described above.

Determine the amount of water in the 'doubly degassed' reaction mixture. A mixture of TOPO/Cd-ODPA was prepared following the 'doubly degassed' protocol using TOPO (2.73 g), $\mathrm{H}_{2}$-ODPA (1.07 g), and CdO (0.205 g). The second degassing was carried out at $150{ }^{\circ} \mathrm{C}, 300 \mathrm{mtorr}$ for $5 \mathrm{~min}$. HYDRANAL-Solvent $2 \mathrm{E}(10 \mathrm{~mL})$ was added to the flask via a syringe at $100{ }^{\circ} \mathrm{C}$. The mixture was cooled to $30{ }^{\circ} \mathrm{C}$ and titrated as described above. The reaction mixture was vigorously shaken to ensure that water adsorbed on the reaction vessel and lower part of the condenser was also titrated.

$\mathbf{H}_{2}{ }^{18} \mathbf{O}$-TOP ${ }^{16} \mathbf{O}$ isotope exchange. To a $25 \mathrm{~mL}$ three-neck flask was added TOPO (2.73 g, $7.06 \mathrm{mmol}), \mathrm{H}_{2}$-ODPA (1.07 g, $\left.3.2 \mathrm{mmol}\right)$, and $\mathrm{CdO}(0.207 \mathrm{~g}, 1.6 \mathrm{mmol})$. The mixture was degassed at $120{ }^{\circ} \mathrm{C}, 300$ mtorr for 20 min. The flask was then filled with Ar and heated to $320{ }^{\circ} \mathrm{C}$ to dissolve $\mathrm{CdO}$ giving a pale yellow solution. The reaction was cooled to $160{ }^{\circ} \mathrm{C}$ and the pressure was reduced to $\sim 500$ mtorr for $33 \mathrm{~min}$. The mixture was then heated to $290{ }^{\circ} \mathrm{C}$ under $\mathrm{Ar}$ and $\mathrm{H}_{2}{ }^{18} \mathrm{O}$ (Isotech, 95\% min ${ }^{18} \mathrm{O}, 56 \mathrm{mg}, 3.1 \mathrm{mmol}$ $\mathrm{H}_{2}{ }^{18} \mathrm{O}$ ) was injected at this temperature via a syringe. Aliquots of the mixture were taken via a syringe and dissolved in methanol before they were analyze by NMR spectroscopy.

Decomposition of TOPSe in TOPO/ $\mathbf{H}_{2}$-ODPA in the absence of $\mathbf{C d}^{2+}$. TOPO ( $1.0 \mathrm{~g}$ ) and $\mathrm{H}_{2}$-ODPA $(0.50 \mathrm{~g})$ were heated to $290{ }^{\circ} \mathrm{C}$ under Ar before TOPSe $(0.537 \mathrm{~g})$ was injected at this temperature. Water $(0.1 \mathrm{~mL})$ was added to the reaction mixture via a syringe at $83 \mathrm{~min}$ after the injection of TOPSe. ${ }^{31} \mathrm{P}$ NMR analysis showed that $7 \%$ and 
$15 \%$ of TOPSe was consumed at $79 \mathrm{~min}$ and $265 \mathrm{~min}$ after the injection of TOPSe, respectively.

\subsection{Characterization of Precursors}

Chalcogen Precursor. Solution of elemental chalcogen in tri-alkylphosphine, such as TOP or TBP, was frequently used as the chalcogen source in the synthesis of group II-VI nanocrystals. It has been known that when chalcogen is dissolved in alkylphosphine, a new compound, phosphine chalcogenide, is formed by the reaction between elemental chalcogen and tri-alkylphosphine in a 1:1 molar ratio. Both MS and NMR data show that phosphine chalcogenide is the only molecule that contains chalcogen atom in this 'solution' of elemental chalcogen. When excess elemental chalcogen is mixed with tri-alkylphosphine, only 1 molar equivalent of chalcogen (relative to phosphine) could be dissolved, which shows that physical dissolution of elemental chalcogen in tri-alkylphosphine and phosphine chalcogenide is negligible at room temperature.

TOPSe, TBPSe, and TBPS were found to be stable in air. No change was observed in the ${ }^{31} \mathrm{P}$ NMR spectrum for a $\mathrm{CDCl}_{3}$ solution of TOPSe that was left in air for 20 days (Figure 2-1). When Cd-ODPA was added to a $\mathrm{CDCl}_{3}$ solution of TOPSe, $<2 \%$ of TOPSe was converted to TOPO after 20 days. Similar results were obtained for $\mathrm{CDCl}_{3}$ solutions of TBPSe and TBPS after they were exposed to air for 5 days.

Metal Precursor. Dissolution of cadmium oxide (CdO) with phosphonic acids has been reported to produce a phosphonic acid complex of cadmium. ${ }^{8,10} \mathrm{~A}$ balanced chemical equation for this reaction is shown below in eq. 2-1. 


$$
\mathrm{CdO}+\mathrm{H}_{2}-\mathrm{ODPA} \rightarrow \mathrm{Cd}-\mathrm{ODPA}+\mathrm{H}_{2} \mathrm{O}
$$

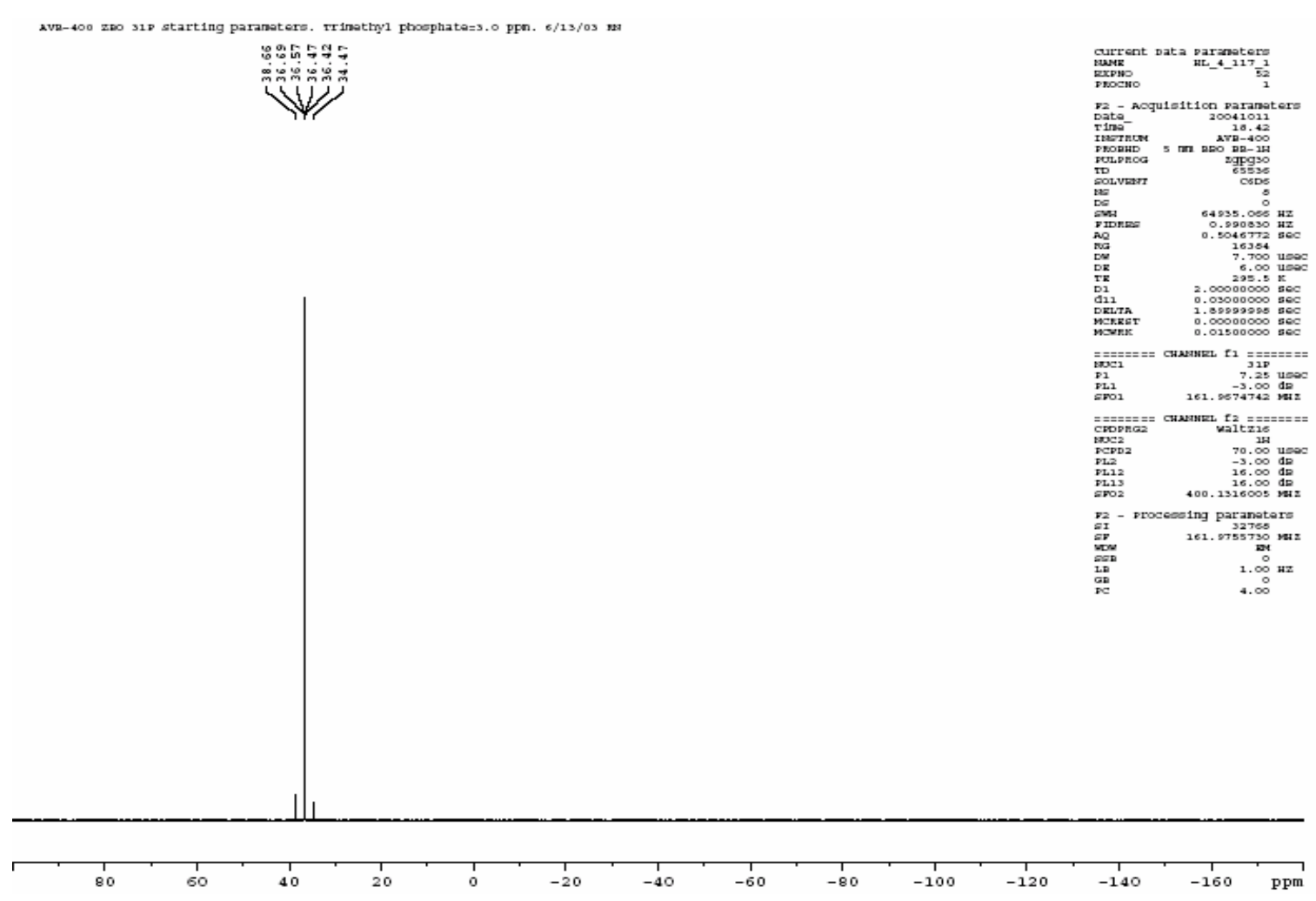

Figure 2-1. ${ }^{31} \mathrm{P}$ NMR spectrum of a $\mathrm{CDCl}_{3}$ solution of TOPSe after it was stored in air for 20 days.

Upon heating cadmium oxide and $\mathrm{H}_{2}$-ODPA in TOPO to $\sim 300{ }^{\circ} \mathrm{C}$, immiscible, colorless droplets form on the walls of the reaction vessel. ${ }^{1} \mathrm{H}$ NMR analysis of these droplets in DMSO- $\mathrm{d}_{6}$ showed no peaks other than an increase in the amount of water impurity in the NMR solvent. The moisture content of this reaction mixture was further analyzed using a Karl-Fischer titration. ${ }^{11,12}$ The titration results indicate that both commercial samples of TOPO and $\mathrm{H}_{2}$-ODPA used in this study have $<0.2 \%(\mathrm{w} / \mathrm{w})$ 
moisture. To confirm the stoichiometry of equation $2-1, \mathrm{CdO}(0.211 \mathrm{~g}, 1.64 \mathrm{mmol})$ was dissolved in a mixture of TOPO $(1.00 \mathrm{~g})$ and $\mathrm{H}_{2}$-ODPA $(1.07 \mathrm{~g}, 3.20 \mathrm{mmol})$ in a tightly closed vial filled with $\mathrm{Ar}$ and the resulting mixture was cooled and dissolved in anhydrous ethanol in a glove box. Karl-Fisher titration showed that the mixture contained $32 \mathrm{mg}$ of water. After subtracting the moisture in TOPO and $\mathrm{H}_{2}$-ODPA, it was found that $28 \mathrm{mg}$ (1.56 mmol; expected: $29.6 \mathrm{mg}, 1.64 \mathrm{mmol})$ of water was produced upon dissolution of the CdO. To test the drying efficiency of the second degassing after decomposing $\mathrm{CdO}$, a reaction mixture was titrated after it had been degassed for $5 \mathrm{~min}$ and only $3 \mathrm{mg}(0.17 \mathrm{mmol}, \sim 10 \%)$ of water remained.

${ }^{31} \mathrm{P}$ NMR spectroscopy was also used to characterize the cadmium $n$ octadecylphosphonate complex (Cd-ODPA). Immediately after dissolving $\mathrm{CdO}$ with $\mathrm{H}_{2-}$ ODPA in TOPO, the mixture was dissolved in $\mathrm{CDCl}_{3}$ giving a clear, viscous solution that became turbid after standing for more than a day. ${ }^{31} \mathrm{P}$ NMR analysis of freshly prepared $\mathrm{CDCl}_{3}$ solutions of Cd-ODPA showed a sharp TOPO resonance and one broad peak at $\delta$ $=25 \mathrm{ppm}(f w h m=10 \mathrm{ppm})$. No resonances for free $\mathrm{H}_{2}$-ODPA were observed. In addition, the acid proton of $\mathrm{H}_{2}$-ODPA could not be located in the ${ }^{1} \mathrm{H}$ NMR spectrum. A similar NMR analysis was performed on the oleic acid complex of cadmium (Cd-OA) prepared by the analogous decomposition of $\mathrm{CdO}$ in $\mathrm{H}-\mathrm{OA}$ using 4:1 molar ratio of $\mathrm{H}-$ OA to CdO. ${ }^{1} \mathrm{H}$ and ${ }^{13} \mathrm{C}$ NMR analysis of Cd-OA showed spectra similar to those of $\mathrm{H}$ OA except that the relative intensity of the acidic proton had dropped $50 \%$ due to the consumption of 2 equivalents of $\mathrm{H}-\mathrm{OA}$ during the dissolution of $\mathrm{CdO}$. The oleic acid complex of zinc (Zn-OA), on the other hand, was much less soluble in $\mathrm{CDCl}_{3}$ and was not analyzed. 
The broad ${ }^{31} \mathrm{P}$ NMR resonance, high viscosity and low solubility of Cd-ODPA in $\mathrm{CDCl}_{3}$ support the idea that this cadmium precursor is a coordination polymer. Single crystal X-ray studies of $\mathrm{Cd}\left(\mathrm{O}_{3} \mathrm{PCH}_{3}\right) \cdot \mathrm{H}_{2} \mathrm{O}$ and $\mathrm{Cd}\left(\mathrm{O}_{3} \mathrm{PC}_{6} \mathrm{H}_{5}\right) \cdot \mathrm{H}_{2} \mathrm{O}$ provide structurally characterized examples of this type of coordination polymer that is composed of layers of cadmium ions bridged together by phosphonate groups. ${ }^{13}$ Unlike Cd-ODPA, Cd-OA is soluble in $\mathrm{CDCl}_{3}$ and gives sharp ${ }^{1} \mathrm{H}$ and ${ }^{13} \mathrm{C}$ NMR resonances, which does not, however, rule out the possibility that this compound also forms a similar coordination polymer or oligomeric structure. Single crystal structures of cadmium acetate and self assembled monolayers of other long chain carboxylic acid complexes of cadmium support the notion that Cd-OA may also be a coordination polymer under our conditions despite its sharp NMR resonances. ${ }^{14}$ The low solubility of the $\mathrm{Zn}-\mathrm{OA}$ in $\mathrm{CDCl}_{3}$ may be due to a polymeric structure in this case.

Phosphonic acid surfactant. $\mathrm{H}_{2}$-ODPA is very insoluble in $\mathrm{CDCl}_{3}$ and hence its ${ }^{31} \mathrm{P}$ NMR spectrum was recorded in methanol- $d_{4} /$ ethanol $(1: 3 \mathrm{v} / \mathrm{v})$ showing one resonance at $\delta=29.4 \mathrm{ppm} . n$-tetradecylphosphonic acid ( $\mathrm{H}_{2}$-TDPA) and $n$-octylphosphonic acid $\left(\mathrm{H}_{2}-\mathrm{OPA}\right)$ are, however, more soluble in $\mathrm{CDCl}_{3}$ and showed singlets at $\delta=38.4$ and 37.7 ppm, respectively. A solution of $\mathrm{H}_{2}-\mathrm{ODPA}$ in $\mathrm{CDCl}_{3}$ could be obtained by dissolving a hot mixture of $\mathrm{H}_{2}$-ODPA and TOPO in $\mathrm{CDCl}_{3}$ or by heating $\mathrm{H}_{2}$-ODPA and TOPO in $\mathrm{CDCl}_{3} . \mathrm{A}{ }^{31} \mathrm{P}$ NMR spectrum of this mixture $\left(\mathrm{H}_{2}-\mathrm{ODPA}:\right.$ TOPO $\left.=1: 2 \mathrm{w} / \mathrm{w}\right)$ contains three peaks at $\delta=50.2 \mathrm{ppm}$ (TOPO), $33.7 \mathrm{ppm}\left(\mathrm{H}_{2}\right.$-ODPA), and $24.1 \mathrm{ppm}$. The resonance at $24.1 \mathrm{ppm}$ does not arise from an impurity of TOPO or ODPA, since it is not observed in a $\mathrm{CDCl}_{3}$ solution of pure TOPO or in a methanol- $d_{4} /$ ethanol solution of pure $\mathrm{H}_{2}$-ODPA. In addition, the intensity of the $24.1 \mathrm{ppm}$ peak is $8 \%$ of the $\mathrm{H}_{2}$-ODPA peak 
intensity in a concentrated sample $\left(50 \mathrm{mg} \mathrm{H}_{2}\right.$-ODPA and $75 \mathrm{mg}$ TOPO in $1 \mathrm{~mL}$ of $\mathrm{CDCl}_{3}$ ) and decreases to $<2 \%$ in a dilute sample ( $5 \mathrm{mg} \mathrm{H}_{2}$-ODPA and $10 \mathrm{mg}$ TOPO in 1 $\mathrm{mL}$ of $\mathrm{CDCl}_{3}$ ). This concentration dependence suggests that the $24.1 \mathrm{ppm}$ peak may arise from a hydrogen bonded complex between TOPO and $\mathrm{H}_{2}$-ODPA or between two or more $\mathrm{H}_{2}$-ODPA molecules. ${ }^{15}$

TOPO. The purity of TOPO was analyzed with ${ }^{31} \mathrm{P}$ NMR spectroscopy. At least three impurities were found in the 99\% pure TOPO purchased from Aldrich (Figure 2-2). The spectrum is very similar to that of a sample of $90 \%$ TOPO from Aldrich. The amount and type of impurity in TOPO depends on the batch and source. However, the ${ }^{31} \mathrm{P}$ NMR spectra of different batch of TOPO usually contain at least two of the three impurities peaks shown in Figure 2-2. Vacuum distillation was not effective in removing all the impurities. 


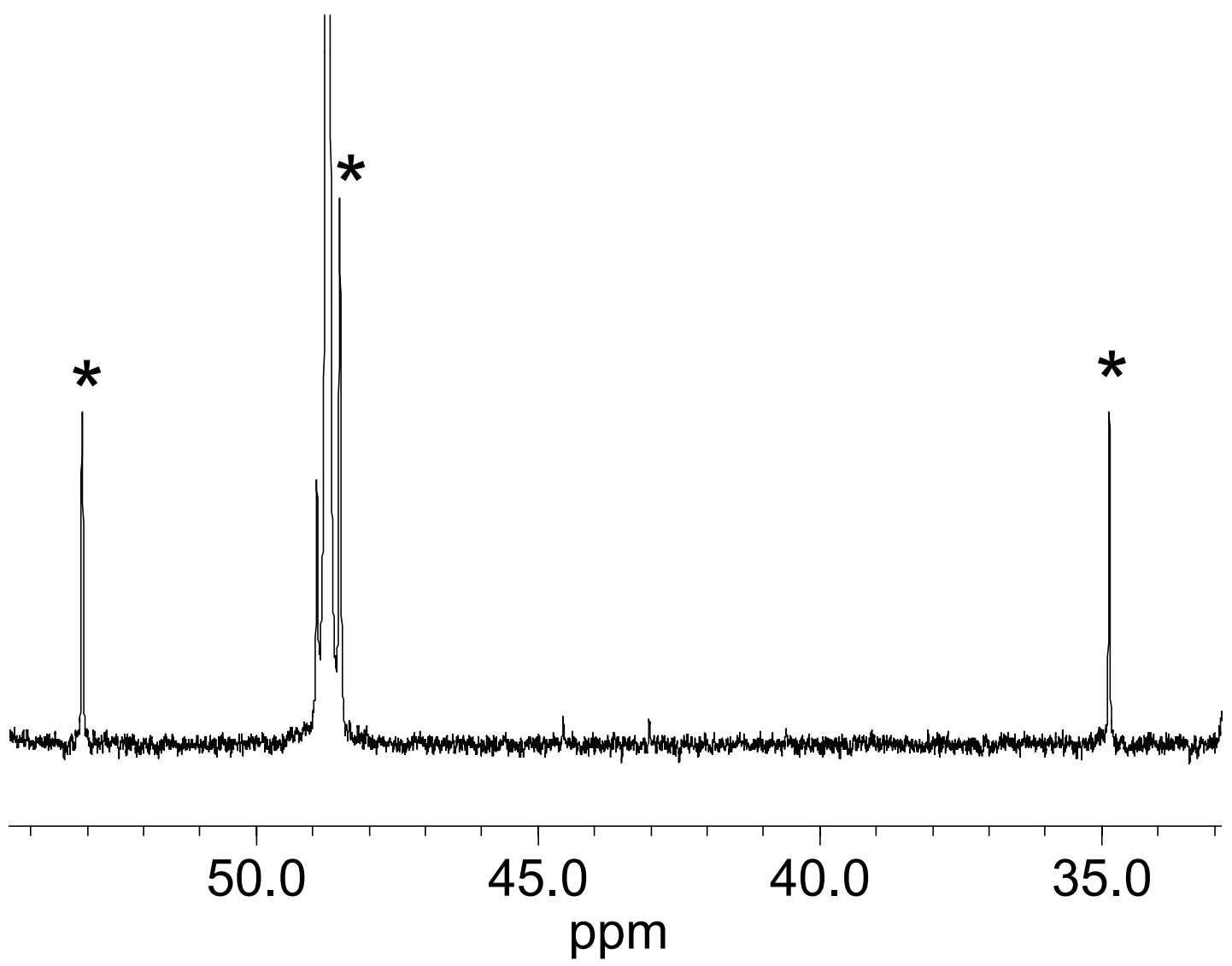

Figure 2-2. ${ }^{31} \mathrm{P}$ NMR spectrum of a sample of $99 \%$ pure TOPO (Aldrich, lot \# 24801MB) used in this study. The spectrum is enlarged to show the impurity peaks $\left(^{*}\right)$. One of the impurity peaks $(\delta=48.5 \mathrm{ppm})$ overlaps with a ${ }^{13} \mathrm{C}$ satellite of TOPO. The purity of TOPO in this sample was estimated to be $93 \%(\mathrm{~mol} / \mathrm{mol})$.

\section{General comments on the ${ }^{31} \mathrm{P}$ NMR spectra of the reagents used in the} synthesis. ${ }^{31} \mathrm{P}$ NMR chemical shifts of Cd-ODPA, as well as the other phosphorus containing reagents, dissolved in $\mathrm{CDCl}_{3}$ are shown in Table 2-1. The chemical shifts of ODPA, TOPO, and TOPSe were found to be sensitive to the presence of Lewis acids and bases. For example, the TOPO resonance shifts downfield from its pure form with added 
$\mathrm{H}_{2}$-ODPA and the major $\mathrm{H}_{2}$-ODPA resonance shifts upfield with added TOPO. The chemical shift of TOPSe was also found to be sensitive to the presence of added Lewis acids. Addition of cadmium 2-ethylhexanoate to a $\mathrm{CDCl}_{3}$ solution of TOPSe caused its ${ }^{31} \mathrm{P}$ NMR resonance to shift to higher ppm and a decrease in the ${ }^{1} J\left({ }^{31} \mathrm{P}-{ }^{77} \mathrm{Se}\right)$. In contrast, however, the addition of cadmium octylphosphonate, ${ }^{16}$ to TOPSe in $\mathrm{CDCl}_{3}$ did not change the chemical shift of TOPSe, nor did it affect the ${ }^{1} J\left({ }^{31} \mathrm{P}-{ }^{77} \mathrm{Se}\right)$. In addition, aliquots taken in the CdSe synthesis using TOPSe and Cd-ODPA in TOPO did not show any change in the chemical shift or ${ }^{1} J\left({ }^{31} \mathrm{P}-{ }^{77} \mathrm{Se}\right)$ of TOPSe when compared to a solution of pure TOPSe in $\mathrm{CDCl}_{3}$.

\begin{tabular}{|c|c|c|}
\hline & $\delta\left({ }^{31} \mathrm{P}\right)[\mathrm{ppm}]$ & ${ }^{1} J\left({ }^{31} \mathrm{P}_{-}{ }^{77} \mathrm{Se}\right)[\mathrm{Hz}]$ \\
\hline TOPO & 48.5 & - \\
\hline $\mathrm{TOPO}+\mathrm{H}_{2}-\mathrm{ODPA}^{a}$ & $50.2,33.7,24.1$ & - \\
\hline TOPS & 48.6 & - \\
\hline TOPSe & 36.4 & 678 \\
\hline $\mathrm{TOPSe}+\mathrm{Cd}^{2+b}$ & 39.3 & 615 \\
\hline TBPS & 48.7 & - \\
\hline TBPSe & 36.8 & 680 \\
\hline ТВРТе & -13.2 & $1655^{e}$ \\
\hline $\mathrm{H}_{2}$-OPA & 37.7 & - \\
\hline $\mathrm{H}_{2}$-TDPA & 38.4 & - \\
\hline $\mathrm{H}_{2}-\mathrm{ODPA}^{d}$ & 29.4 & \\
\hline Cd-ODPA & 25 & - \\
\hline
\end{tabular}

Table 2-1. ${ }^{31} \mathrm{P}$ NMR chemical shifts of the phosphorus containing surfactants and precursors. All spectra were taken in $\mathrm{CDCl}_{3}$ at room temperature and referenced to tri- 
methylphosphate $(\delta=3.0 \mathrm{ppm}){ }^{a}$ Prepared by heating $\mathrm{H}_{2}-\mathrm{ODPA}$ and TOPO $(1: 2 \mathrm{w} / \mathrm{w})$ under $\mathrm{Ar}$ to $290{ }^{\circ} \mathrm{C}$ and then dissolving the mixture in $\mathrm{CDCl}_{3}$. ${ }^{b}$ Cadmium 2ethylhexanoate. ${ }^{c 1} J\left({ }^{31} \mathrm{P}_{-}{ }^{125} \mathrm{Te}\right) .{ }^{d}$ Solvent: $\mathrm{CD}_{3} \mathrm{OD} / \mathrm{CH}_{3} \mathrm{CH}_{2} \mathrm{OH}(1: 3 \mathrm{v} / \mathrm{v})$.

\subsection{Characterization of Reaction Products}

Phosphine oxide. In situ ${ }^{31} \mathrm{P}$ NMR spectroscopy was used to monitor the reaction of Cd-OA or Zn-OA with TBPSe in a hydrocarbon solvent under vacuum in flame sealed NMR tubes. In both the case of $\mathrm{Cd}$ and $\mathrm{Zn}$ the disappearance of TBPSe proceeds with the formation of TBPO, which was verified by the addition of an authentic sample to the NMR sample as well as by detection with mass spectroscopy (Figure 2-3). In several examples, the total concentration of TBPSe and TBPO was constant throughout the reaction (within $\pm 2 \%$ ), versus an internal standard (ethylphosphonic acid diethyl ester), which requires that TBPO is the only major product of TBPSe decomposition. A fast atom bombardment - mass spectrum (FAB-MS) of the crude reaction mixture shows a strong peak at $m / z=219$ corresponding to the $[\mathrm{TBPO}+\mathrm{H}]^{+}$ion (Figure $\left.2-4\right)$. Similar to the synthesis in hydrocarbon solvents, a synthesis of CdSe, in TOPO solvent using TBPSe as the Se precursor, also afforded TBPO as one of the major reaction products. Analysis of a crude reaction mixture using FAB-MS showed a strong peak at $m / z=219$ that corresponds to the $\left[\mathrm{TBPO}+\mathrm{H}^{+}\right]$ion (Figure 2-5). 


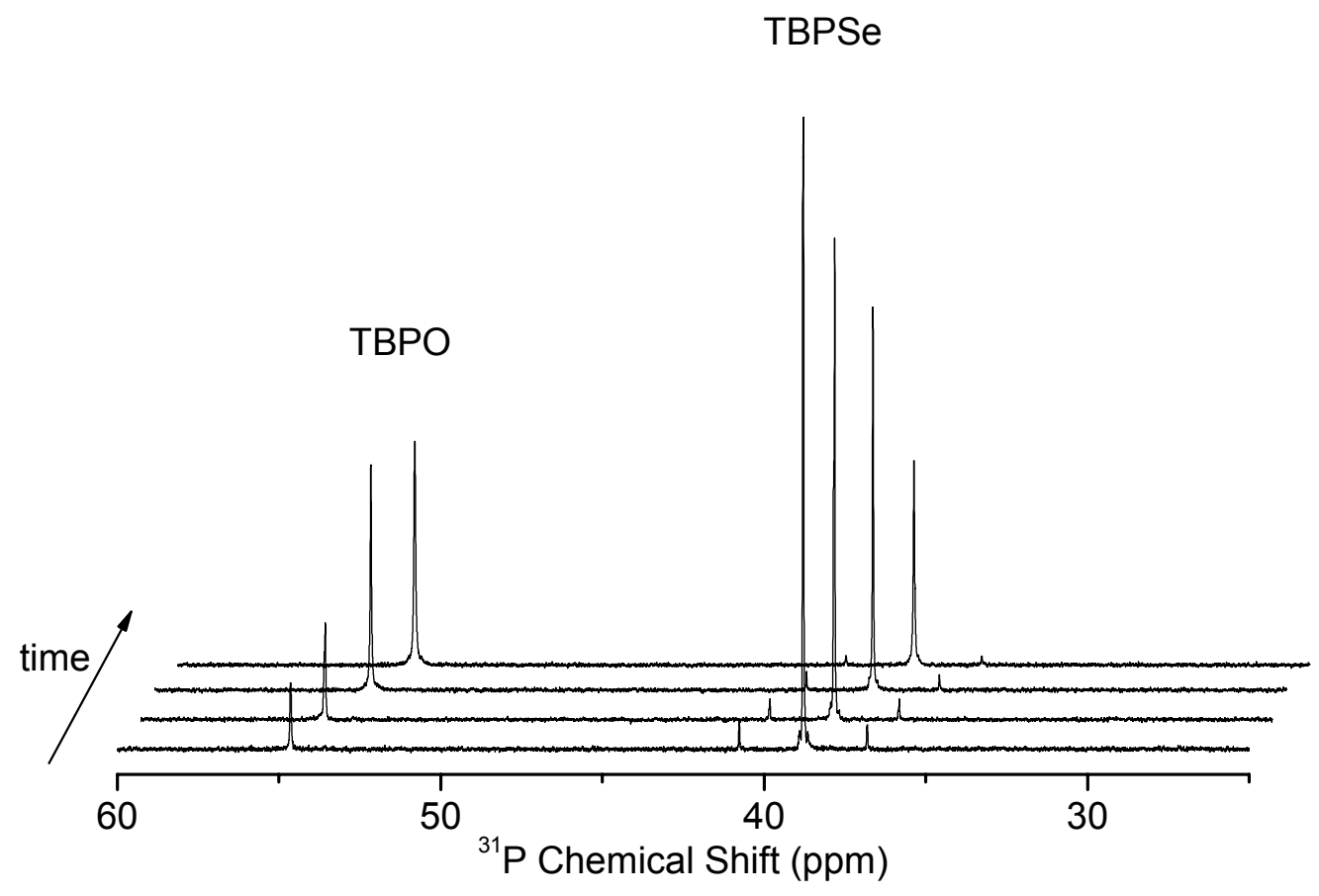

Figure 2-3. Time evolution of the ${ }^{31} \mathrm{P}$ NMR spectrum of the reaction between TBPSe and Cd-OA in $n$-nonane- $d_{20}$ at $380 \mathrm{~K}$. Spectra were collected at $0,140,1110$, and 2145 seconds respectively. 


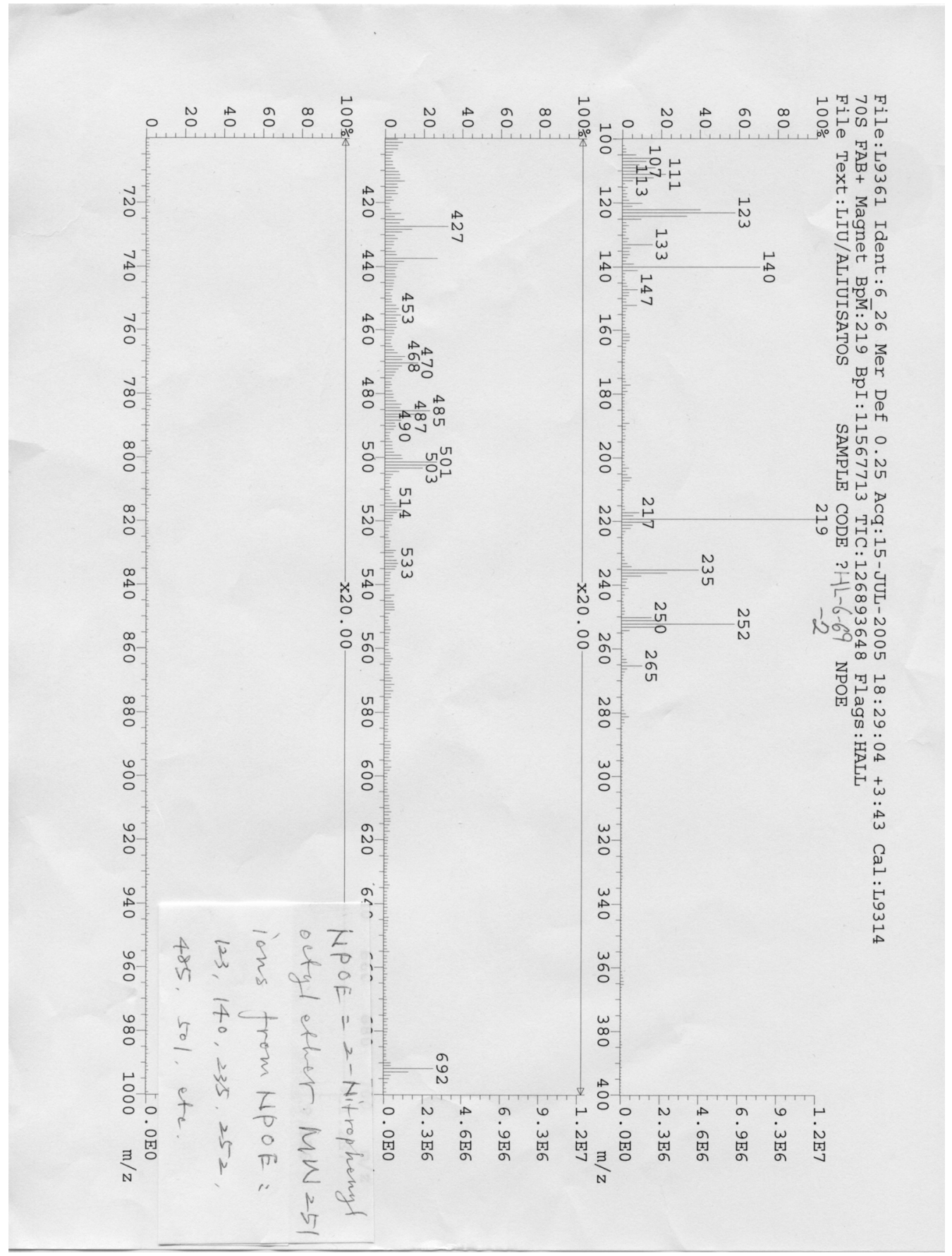

Figure 2-4. FAB-MS spectrum of the reaction between $\mathrm{Cd}-\mathrm{OA}$ and TBPSe. $[\mathrm{TBPO}+\mathrm{H}]^{+}$ $(m / z=219)$ 


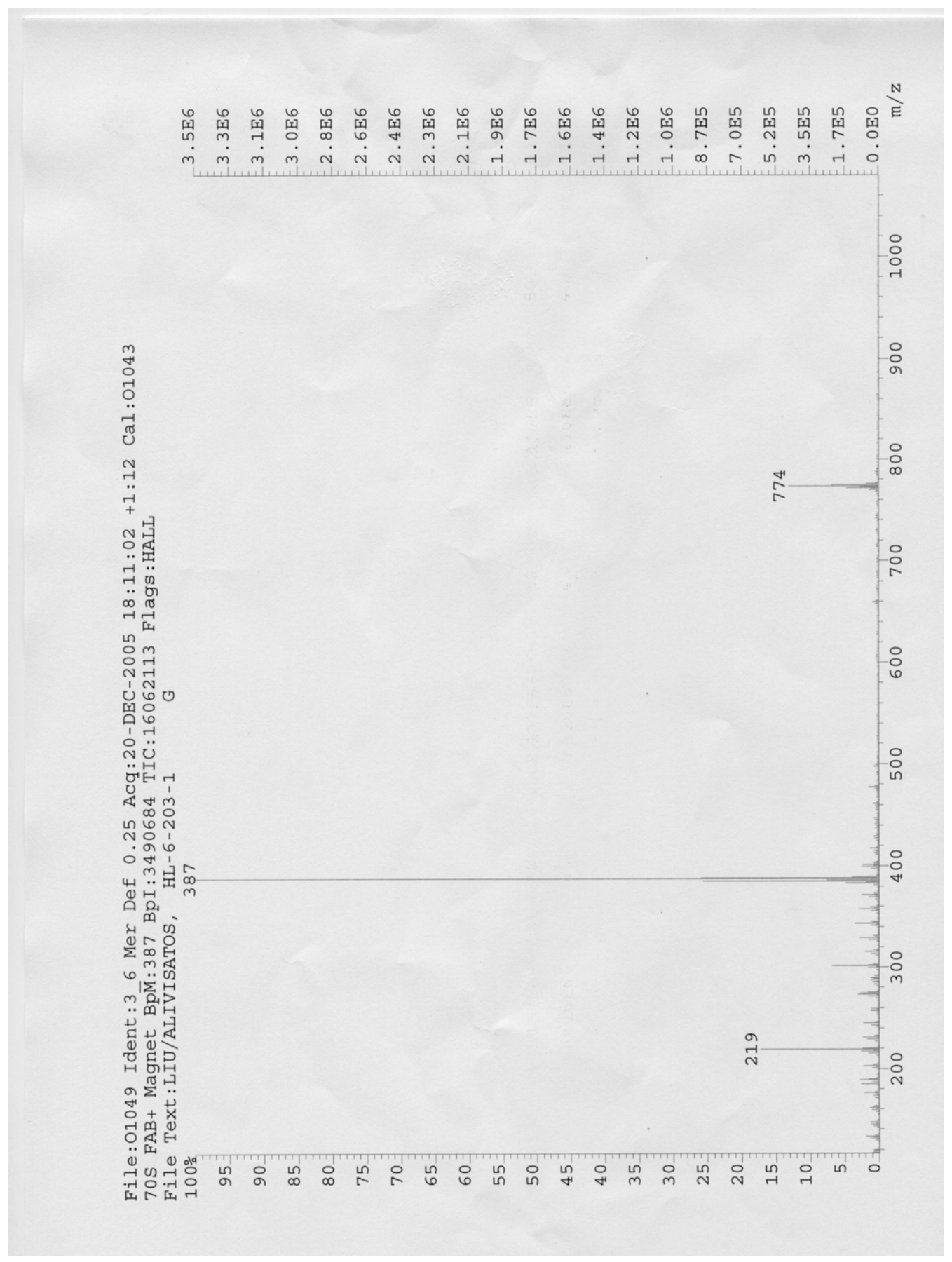

Figure 2-5. FAB-MS of the reaction between Cd-ODPA and TBPSe in TOPO. $[\mathrm{TBPO}+\mathrm{H}]^{+}(m / z=219),[\mathrm{TOPO}+\mathrm{H}]^{+}(m / z=387)$, and $[2 \mathrm{TOPO}+\mathrm{H}]^{+}(m / z=774)$ 
Carboxylic acid anhydride. In addition to the conversion of TBPSe to TBPO, the formation of oleic acid anhydride $\left((\mathrm{OA})_{2} \mathrm{O}\right)$ was observed in the ${ }^{13} \mathrm{C}$ NMR spectrum of reactions between $\mathrm{Zn}-\mathrm{OA}$ or $\mathrm{Cd}-\mathrm{OA}$ and $\mathrm{TBPSe}$. In situ analysis of the reaction products showed new peaks at $\delta=168.1,129.8,129.4$ and 35.1 ppm not present before heating the mixture. Comparison of this spectrum with an authentic sample of $(\mathrm{OA})_{2} \mathrm{O}$ confirmed the assignment (Figure 2-6). Control experiments showed that heating the CdOA stock solution (at $120^{\circ} \mathrm{C}$ in degassed and sealed NMR tube for 2 hours) or pure $\mathrm{H}$ OA (at $120^{\circ} \mathrm{C}$ under dynamic vacuum for 2 hours) did not produce detectable amount of $(\mathrm{OA})_{2} \mathrm{O}$ by ${ }^{1} \mathrm{H}$ and ${ }^{13} \mathrm{C}$ NMR. In several runs, the appearance of the $\alpha$-methylene peak $\left({ }^{1} \mathrm{H}\right.$ NMR $)$ of $(\mathrm{OA})_{2} \mathrm{O}(\delta=2.50 \mathrm{ppm})$ matches the disappearance of the ${ }^{31} \mathrm{P}$ NMR resonance of TBPSe (within $\pm 2 \%$ ). Similar observations were made in the synthesis of other metal chalcogenides as well as in the reactions that were conducted in the presence of added trialkylphosphine (Table 2-2). 


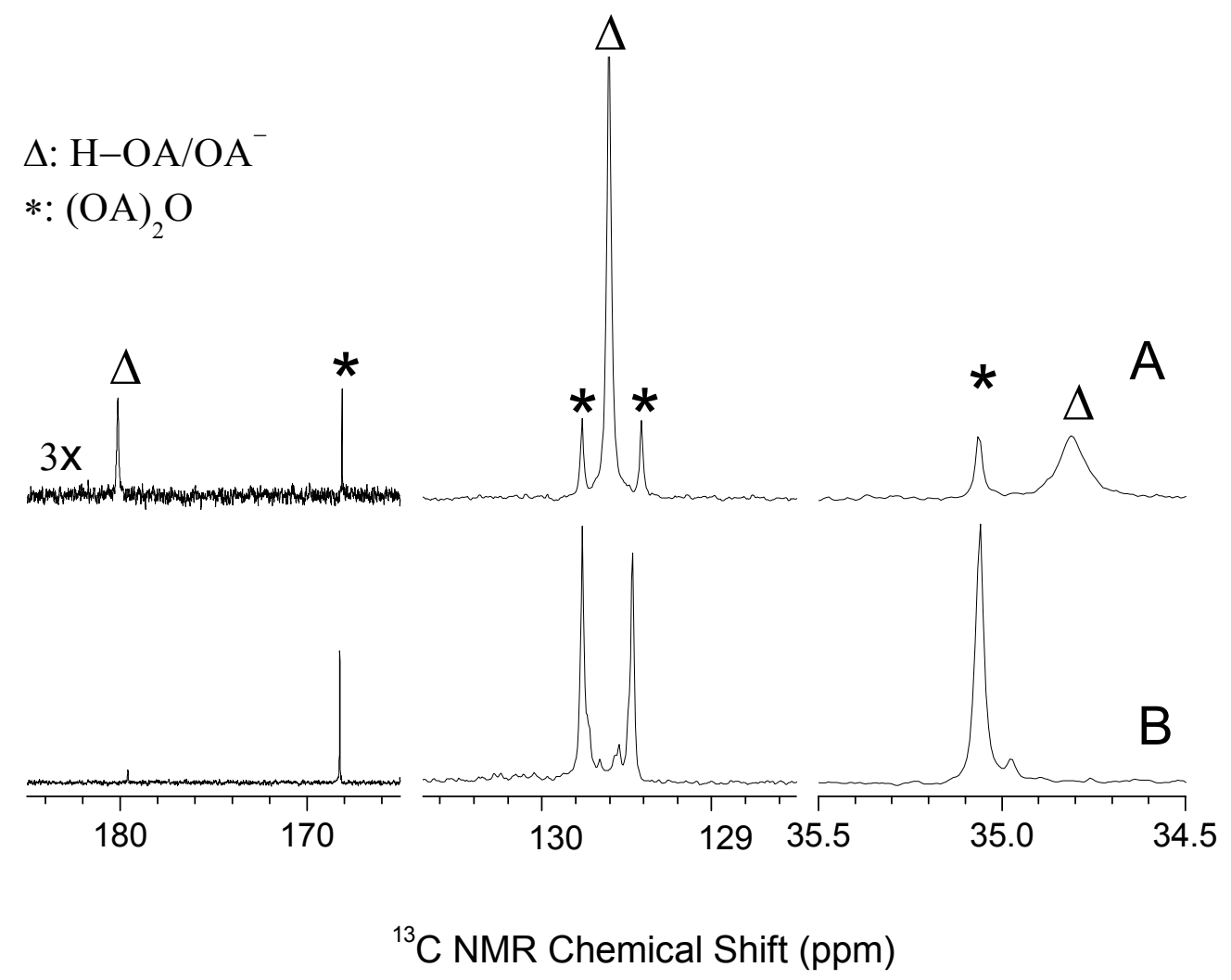

Figure 2-6. ${ }^{13} \mathrm{C}$ NMR spectrum of $(\mathbf{A})$ the reaction between $\mathrm{Cd}-\mathrm{OA}$ and TBPSe and (B) an authentic sample of $(\mathrm{OA})_{2} \mathrm{O}$ in $n$-nonane- $d_{20}$. *: $(\mathrm{OA})_{2} \mathrm{O}$ peaks, 168.1 ppm: ($\left.\mathrm{CH}_{2} \mathbf{C O}\right)_{2} \mathrm{O}, 129.8$ and 129.4 ppm: $-\mathbf{C H}=\mathbf{C H}-, 35.1$ ppm $\left(-\mathbf{C H}_{2}-\mathrm{COO}\right)_{2} \mathrm{O} . \Delta: \mathrm{H}-\mathrm{OA}$ peaks, 180.1 ppm: $-\mathrm{CH}_{2} \mathbf{C O O H}, 129.6$ ppm: $-\mathbf{C H}=\mathbf{C H}-, 34.8$ ppm $-\mathbf{C H}_{2}-\mathrm{COOH}$. The small peak at $\sim 180 \mathrm{ppm}$ in the $(\mathrm{OA})_{2} \mathrm{O}$ spectrum is due to the presence of an H-OA impurity in the commercial sample. 


\begin{tabular}{|c|c|c|c|}
\hline & Precursors & Surfactant/Solvent & Identified Products (characterization) \\
\hline CdSe & TBPSe/Cd-ODPA & $\mathrm{H}_{2}$-ODPA/TOPO & TBPO (FAB-MS) \\
\hline CdSe & TOPSe/Cd-ODPA & $\mathrm{H}_{2}$-ODPA/TOPO & ODPA anhydride $\left({ }^{31} \mathrm{P} N M R,{ }^{31} \mathrm{P}_{-}{ }^{31} \mathrm{P}\right.$ COSY $)$ \\
\hline CdSe & TBPSe/Cd-ODPA & $\mathrm{H}_{2}$-ODPA/TOPO, TBP & ODPA anhydride ( ${ }^{31} \mathrm{P}$ NMR) \\
\hline CdSe & TBPSe/Cd-OA ${ }^{a}$ & $\mathrm{H}-\mathrm{OA} / \mathrm{C}_{10} \mathrm{D}_{22}$ & TBPO $\left({ }^{31} \mathrm{P}\right.$ NMR $),(\mathrm{OA})_{2} \mathrm{O}\left({ }^{13} \mathrm{C}\right.$ NMR $)$ \\
\hline CdSe & TBPSe/Cd-OA ${ }^{a, b}$ & $\mathrm{H}-\mathrm{OA} / \mathrm{C}_{10} \mathrm{D}_{22}, \mathrm{TBP}$ & TBPO $\left({ }^{31} \mathrm{P}\right.$ NMR $),(\mathrm{OA})_{2} \mathrm{O}\left({ }^{13} \mathrm{C} \mathrm{NMR}\right)$ \\
\hline $\mathrm{CdS}$ & TBPS/Cd-OA ${ }^{a}$ & $\mathrm{H}-\mathrm{OA} / \mathrm{C}_{10} \mathrm{D}_{22}$ & TBPO $\left({ }^{31} \mathrm{P}\right.$ NMR, FAB-MS $\left.),(\mathrm{OA})\right)_{2} \mathrm{O}\left({ }^{13} \mathrm{C}\right.$ NMR $)$ \\
\hline CdTe & $\mathrm{TBPTe} / \mathrm{Cd}-\mathrm{OA}^{a}$ & $\mathrm{H}-\mathrm{OA} / \mathrm{C}_{10} \mathrm{D}_{22}$ & TBPO $\left({ }^{31} \mathrm{P} N M R\right),(\mathrm{OA})_{2} \mathrm{O}\left({ }^{13} \mathrm{C} \mathrm{NMR}\right)$ \\
\hline $\mathrm{ZnS}$ & TBPS/Zn-OA ${ }^{a}$ & $\mathrm{H}-\mathrm{OA} / \mathrm{ODE}$ & TBPO $\left({ }^{31} \mathrm{P}\right.$ NMR, FAB-MS $),(\mathrm{OA}){ }_{2} \mathrm{O}\left({ }^{13} \mathrm{C}\right.$ NMR $)$ \\
\hline $\mathrm{ZnSe}$ & TBPSe/Zn-OA ${ }^{a}$ & $\mathrm{H}-\mathrm{OA} / \mathrm{ODE}$ & TBPO $\left({ }^{31} \mathrm{P}\right.$ NMR, FAB-MS $),(\mathrm{OA})_{2} \mathrm{O}\left({ }^{13} \mathrm{C}\right.$ NMR $)$ \\
\hline ZnTe & TBPTe/Zn-OA ${ }^{a, c}$ & $\mathrm{H}-\mathrm{OA} / \mathrm{C}_{10} \mathrm{D}_{22}, \mathrm{TBP}$ & TBPO $\left({ }^{31} \mathrm{P} N M R\right),(\mathrm{OA})_{2} \mathrm{O}\left({ }^{13} \mathrm{C} \mathrm{NMR}\right)$ \\
\hline
\end{tabular}

Table 2-2. Spectroscopically characterized reaction products from the synthesis of group II-VI nanocrystals. COSY: correlation spectroscopy. $\mathrm{C}_{10} \mathrm{D}_{22}: n$-decane- $\mathrm{d}_{22} \cdot{ }^{a}$ In degassed and flame sealed NMR tube. ${ }^{b}$ TBP:TBPSe $=1: 1(\mathrm{~mol} / \mathrm{mol}) .{ }^{c}$ TBP:TBPTe $=1: 1(\mathrm{w} / \mathrm{w})$.

Phosphonic acid anhydride. Aliquots from reactions of Cd-ODPA with TOPSe or TBPSe in TOPO show the disappearance of TOPSe and the appearance of ${ }^{31} \mathrm{P}$ NMR resonances in the range of $\delta=10$ - $33 \mathrm{ppm}$. Immediately after injecting TOPSe into a hot mixture of TOPO and Cd-ODPA, several multiplets appear in the ${ }^{31} \mathrm{P}$ NMR spectrum at $\delta$ $=32,29,28$, and 24 ppm (Figure 2-7). At longer reaction times, additional multiplets could be observed in the range of $\delta=10-23 \mathrm{ppm} .{ }^{31} \mathrm{P}$ homonuclear correlation spectroscopy (COSY) shows that the splitting pattern of these peaks can be explained by ${ }^{31} \mathrm{P}-{ }^{31} \mathrm{P}$ through-bond coupling $\left(J^{31} \mathrm{P}-{ }^{31} \mathrm{P}=35-38 \mathrm{~Hz}\right)$ (Figure 2-8). The chemical shift 
and magnitude of the coupling constants are in the range of reported values for other anhydrides of phosphonic acids $\left(\delta=20-30 \mathrm{ppm}\right.$ and ${ }^{2} J^{31} \mathrm{P}-{ }^{31} \mathrm{P}=25-49 \mathrm{~Hz}$, for pyrophosphonic $\operatorname{acid}^{17}$ and tris(tert-butyl)triphosphonic $\operatorname{acid}^{18}$ ) suggesting similar anhydride linkages may explain the coupling patterns observed in our spectra. Further confirmation of an anhydride linkage being responsible for the multiplets was provided by addition of water to the reaction mixture at the reaction temperature. Upon adding water, the multiplets in the ${ }^{31} \mathrm{P}$ NMR spectrum were immediately replaced by two peaks at $\delta=34$ and $24 \mathrm{ppm}$, which are in the range of the resonances observed for $\mathrm{H}_{2}$-ODPA in the presence of TOPO (Figure 2-9). In addition, the ${ }^{1} \mathrm{H}$ NMR showed an increase in the amount of the acid proton. 


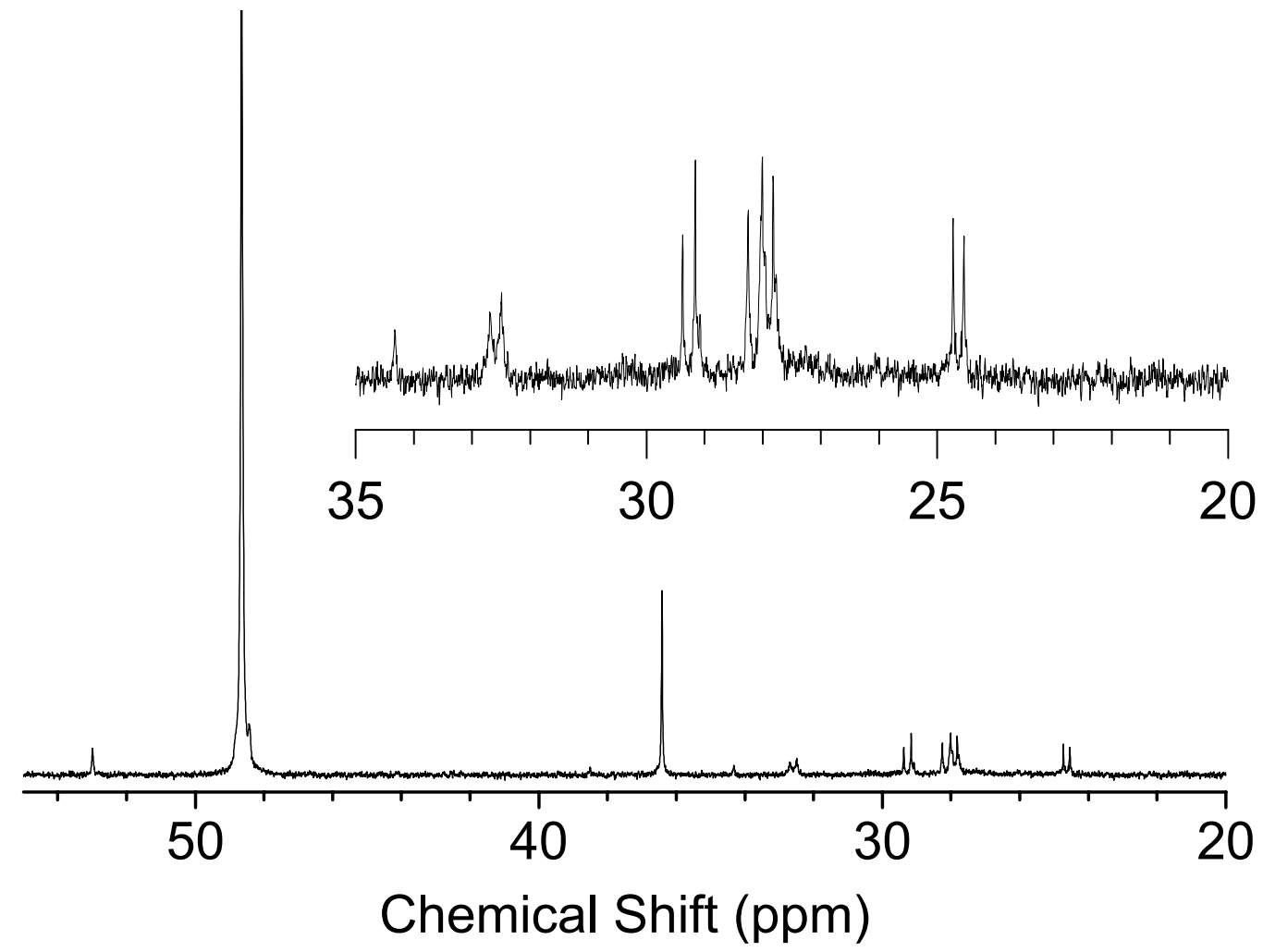

Figure 2-7. ${ }^{31} \mathrm{P}$ NMR spectrum of the a CdSe synthesis in TOPO. The crude reaction mixture was dissolved in $\mathrm{CDCl}_{3}$. Inset shows magnified view of 20-35 ppm of the spectrum. TOPO, $\delta=48.7 \mathrm{ppm}$, TOPSe, $\delta=36.3 \mathrm{ppm}$. 


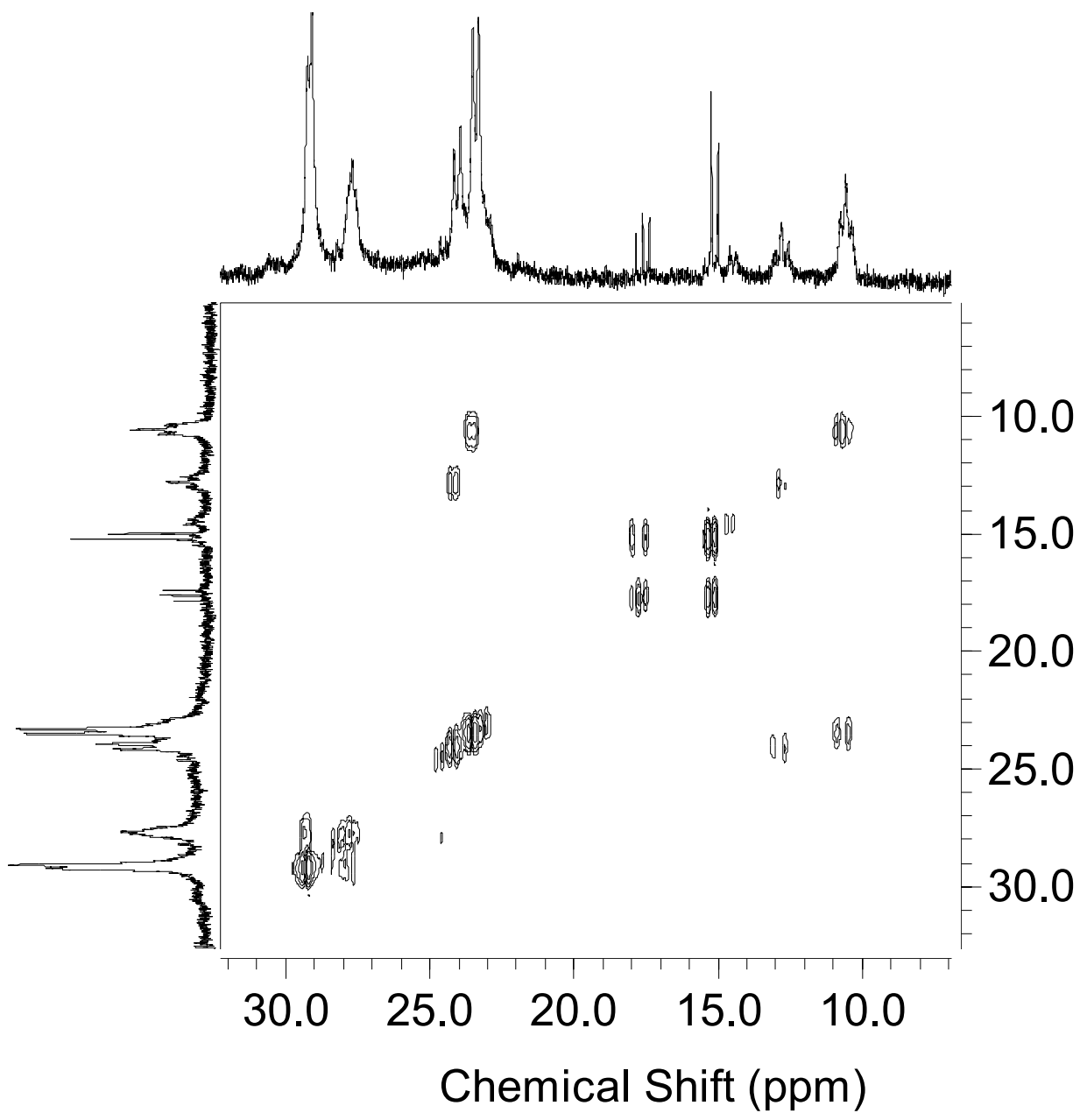

Figure 2-8. ${ }^{31} \mathrm{P}_{-}{ }^{31} \mathrm{P}$ COSY spectrum of a CdSe synthesis. Peaks of TOPO and TOPSe give no cross peak and are not shown. The crude reaction mixture was dissolved in $\mathrm{CDCl}_{3}$ and flame sealed in an NMR tube under Ar. 

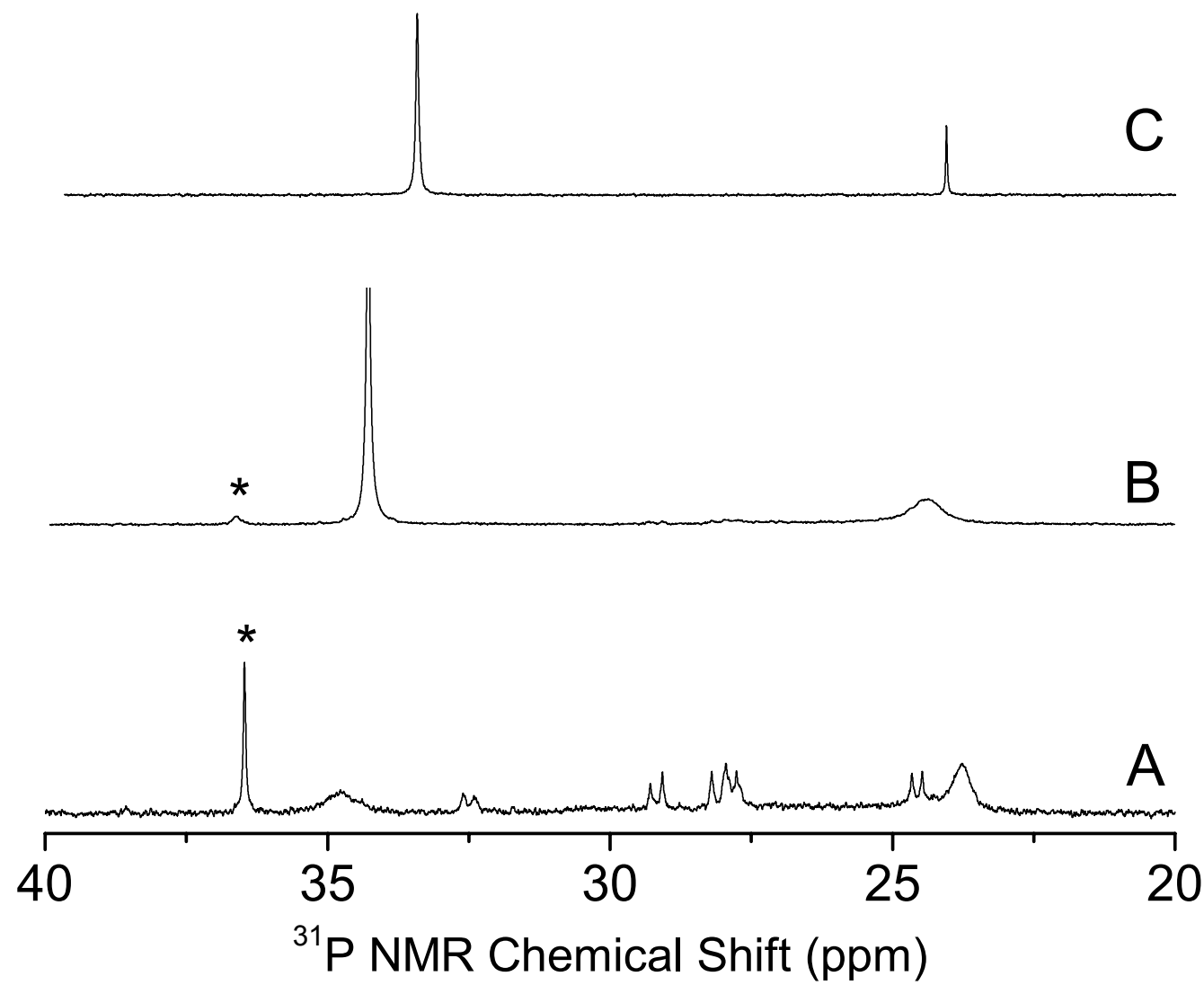

Figure 2-9. The effect of added water on the ${ }^{31} \mathrm{P}$ NMR spectrum of a TOPSe and CdODPA reaction mixture in TOPO. A) before and $\mathrm{B}$ ) after adding water to the reaction. C) ${ }^{31} \mathrm{P}$ NMR of a $2: 1 \mathrm{w} / \mathrm{w}$ mixture of TOPO and $\mathrm{H}_{2}$-ODPA. * TOPSe peak. 
To further clarify the role of water in the synthesis of CdSe in TOPO, control experiments were carried out by adding water $(100 \mathrm{mg})$ to the reaction after the second degassing but before the TOPSe injection. After injection aliquots of this reaction show the appearance of ${ }^{31} \mathrm{P}$ multiplets at $\delta=20-30 \mathrm{ppm}$ that are replaced by two peaks at $\delta=$ 34 and $24 \mathrm{ppm}$ at longer times ( 1 hour). To confirm that the added water is indeed accessible to the TOPO solution at the reaction temperature, we also carried out a control experiment in which $\mathrm{H}_{2}{ }^{18} \mathrm{O}$ (Isotech, 95\% ${ }^{18} \mathrm{O}$ ) was injected to a mixture of TOPO and Cd-ODPA at $290{ }^{\circ} \mathrm{C}$. Both FAB-MS and ${ }^{31} \mathrm{P}$ NMR showed rapid formation of ${ }^{18} \mathrm{O}$ substituted TOPO (Figure 2-10), which is consistent with an oxygen exchange reaction between $\mathrm{H}_{2}{ }^{18} \mathrm{O}$ and TOPO. ${ }^{19}$ These experiments ambiguously demonstrated that water not only exists in the mixture of TOPO and Cd-ODPA at $290{ }^{\circ} \mathrm{C}$ but also interacts chemically with TOPO, and presumably, other molecules.

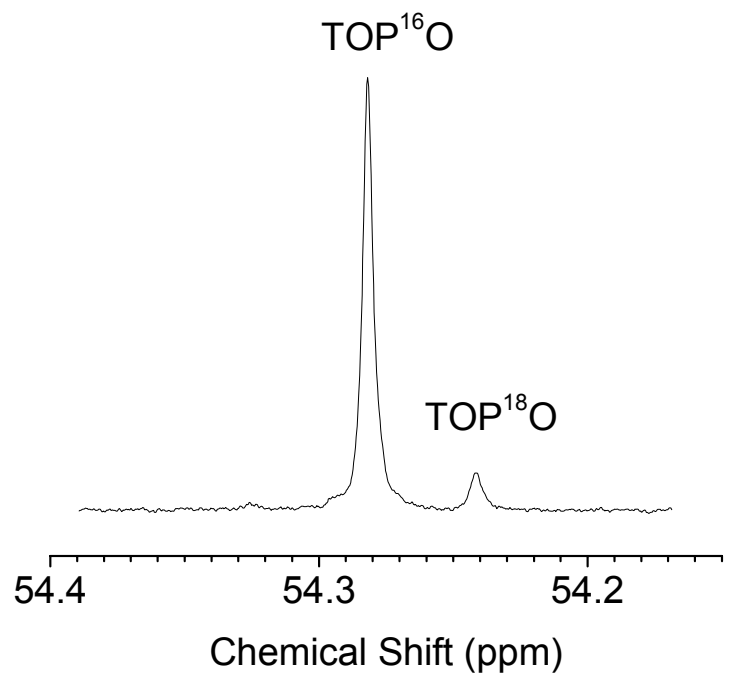

Figure 2-10. ${ }^{31} \mathrm{P}$ NMR $\left(\mathrm{CH}_{3} \mathrm{OH} / \mathrm{CDCl}_{3}\right)$ of TOPO after injecting $\mathrm{H}_{2}{ }^{18} \mathrm{O}$ to a mixture of TOPO and Cd -ODPA at $290^{\circ} \mathrm{C}$. This sample was taken 24 min after the injection of $\mathrm{H}_{2}{ }^{18} \mathrm{O}$. 


\section{References}

(1) Peng, X. G.; Wickham, J.; Alivisatos, A. P. J. Am. Chem. Soc. 1998, 120, $5343-5344$.

(2) Peng, X. G.; Manna, L.; Yang, W. D.; Wickham, J.; Scher, E.; Kadavanich, A.; Alivisatos, A. P. Nature 2000, 404, 59-61.

(3) Manna, L.; Scher, E. C.; Alivisatos, A. P. J. Am. Chem. Soc. 2000, 122, 12700-12706.

(4) Peng, Z. A.; Peng, X. G. J. Am. Chem. Soc. 2001, 123, 1389-1395.

(5) Manna, L.; Milliron, D. J.; Meisel, A.; Scher, E. C.; Alivisatos, A. P. Nature Mater. 2003, 2, 382-385.

(6) Kanaras, A. G.; Sonnichsen, C.; Liu, H. T.; Alivisatos, A. P. Nano Lett. 2005, 5, 2164-2167.

(7) Ammann, C.; Meier, P.; Merbach, A. E. J. Magn. Res. 1982, 46, 319-321.

(8) Peng, Z. A.; Peng, X. G. J. Am. Chem. Soc. 2002, 124, 3343-3353.

(9) Yu, W. W.; Wang, Y. A.; Peng, X. G. Chem. Mater. 2003, 15, 4300-4308.

(10) Peng, Z. A.; Peng, X. G. J. Am. Chem. Soc. 2001, 123, 183-184.

(11) Verhoef, J. C.; Barendrecht, E. J. Elec. Chem. 1977, 75, 705-717.

(12) Verhoef, J. C.; Barendrecht, E. Ana. Chim. Acta 1977, 94, 395-403.

(13) Cao, G.; Lynch, V. M.; Yacullo, L. N. Chem. Mater. 1993, 5, 1000-1006.

(14) Harrison, W.; Trotter, J. J. Chem. Soc., Dalton Trans. 1972, 956.

(15) Clearfield, A.; Sharma, C. V. K.; Zhang, B. P. Chem. Mater. 2001, 13, 3099-3112. 
(16) This was prepared by adding $\mathrm{CdMe}_{2}$ to a $\mathrm{CDCl}_{3}$ solution of $n$ octylphosphonic acid at room temperature in a glove box.

(17) Ohms, G.; Grossmann, G.; Schwab, B.; Schiefer, H. Phosphorus Sulfur and Silicon and the Related Elements 1992, 68, 77-89.

(18) Diemert, K.; Kuchen, W.; Poll, W.; Sandt, F. Eur. J. Inorg. Chem. 1998, 361-366.

(19) Denney, D. B.; Tsolis, A. K.; Mislow, K. J. Am. Chem. Soc. 1964, 86, 4486-4487. 


\section{Chapter 3}

\section{Kinetics and Mechanism of the Synthesis of}

\section{Group II-VI Nanocrystals}

Reproduced in part with permission from "Liu, H.; Owen, J. S.; Alivisatos, A. P. "Mechanistic Study of Precursor Evolution in Colloidal Group II-VI Semiconductor Nanocrystal Synthesis" J. Am. Chem. Soc. 2007, 129, 305-312, Copyright 2007 American Chemical Society.

\subsection{Introduction}

The growth mechanism of nanocrystal is one of the several unanswered key questions in the field of nanocrystal synthesis. Such information would greatly help optimizing current nanocrystal synthesis and designing of new synthetic protocols. Previous studies toward this goal are heavily focused on following the time evolution of nanocrystal size and shape by using TEM, UV-Vis, and PL techniques. Peng and Mulvaney have studied the time evolution of CdSe nanocrystals in TOPO/ODPA and

ODE/OA mixtures, respectively. ${ }^{1-3}$ Their studies have concluded that the nucleation and growth of CdSe nanocrystal are well separated. Peng explained the shape evolution of CdSe nanorod by using a diffusion-limited growth model. However, since the techniques 
used in these studies only probe the dynamics of nanocrystals, little to no information is obtained about the molecular mechanism of nanocrystal nucleation and growth. For example, the term 'monomer' is frequently used to refer to the basic growth unit of nanocrystal. However, not much is known about the structure of monomer and how monomer is produced and converted into nanocrystals.

Our approach to characterize the growth kinetics of nanocrystal and study the growth mechanism is very different from these nanocrystal-based ones. With the information presented in the last chapter, it is now possible to accurately measure the kinetics of phosphine chalcogenides decay and analyze the chemistry associated with the precursor decomposition process. Our results suggest that phosphine chalcogenide reacts with the metal precursor to produce a soluble form of semiconductor, which we believe is the 'monomer'.

\subsection{Kinetics of Phosphine Chalcogenide Cleavage.}

Reaction kinetics of the CdSe and CdS syntheses were studied by following the concentration of phosphine chalcogenide using ${ }^{31} \mathrm{P}$ NMR spectroscopy. The decay of phosphine chalcogenide can be fit to a single exponential decay (Figure 3-1) and the corresponding $k_{\mathrm{obs}}$ extracted from the fit (Table 3-1). The cleavage rates of phosphine chacogenides increase in the order $\mathrm{S}<\mathrm{Se}<\mathrm{Te}$ and $\mathrm{Zn}<\mathrm{Cd}$ by comparing their respective $k_{\mathrm{obs}}$ or conversion vs. time. It was also observed that Cd-OA reacts more rapidly than does Cd-ODPA and both TBPSe and TOPSe are much more reactive than the sterically hindered $i$-TPPSe. A control reaction showed that in the absence of Cd-OA or Cd-ODPA, TOPSe still decomposed in a mixture of TOPO and $\mathrm{H}_{2}$-ODPA at $290{ }^{\circ} \mathrm{C}$ 
(Table 3-1 \#8), although at a rate two orders of magnitude slower than in the presence of Cd-OA or Cd-ODPA. Activation parameters for TBPSe decay in the presence of Cd-OA were determined from the temperature dependence of the exponential fits to the decay curve $\left(\Delta H^{*}=(62.0 \pm 2.8) \mathrm{kJ} \cdot \mathrm{mol}^{-1}, \Delta S^{\sharp}=-(145 \pm 8) \mathrm{J} \cdot \mathrm{mol}^{-1} \cdot \mathrm{K}^{-1}\right.$, Figure 3-2).

The effect of temperature and concentration of precursor on the decay kinetics of phosphine selenide was tabulated in table 3-2 and 3-3. The kinetics behavior of the reactions conducted in ODE using oleic acid as the surfactant is very different from that of the reactions conducted in TOPO using phosphonic acid as the surfactant. In the TOPO/ODPA based reactions, the rate of phosphine selenide decay highly depends on the ODPA/Cd ratio and is not sensitive to the $\mathrm{Se} / \mathrm{Cd}$ ratio. High ODPA/Cd ratio is associated with rapid decay of phosphine selenide. In contrast, in the ODE/OA based reactions, the opposite is true. In these reactions, the $\mathrm{Cd} / \mathrm{OA}$ ratio does not affect the reaction kinetics very much. The $\mathrm{Se} / \mathrm{Cd}$ ratio, on the contrary, greatly changes the reaction kinetics. The $k_{\mathrm{obs}}$ of phosphine selenide decay almost increases linearly with increasing Se/Cd ratio. These differences in the reaction kinetics suggest that the rate limiting step of phosphine selenide cleavage is different in the two types of reaction. It is likely that this difference may arise from the different structure of the cadmium precursors. The Cd-ODPA complex used in the TOPO/ODPA synthesis is polymeric and less reactive than the Cd-OA complex used in the ODE/OA synthesis. One possible explanation for the observed difference in the reaction kinetics is that excess free surfactant helps to break the Cd-ODPA complex into certain molecular Cd complex, which has a higher activity than its polymeric counterparts. 
For all the reactions carried out in TOPO under anhydrous conditions, the conversion of trialkyl-phosphine chalcogenide approaches partial conversion $(\sim 80 \%)$ even though Cd was used in excess $(\sim 10 \%)$ in most of these reactions. ${ }^{1}$ The conversion of TBPSe is even lower $(\sim 70 \%)$ when reacted with Cd-OA in a non-coordinating solvent at relatively low temperature $(360 \mathrm{~K}-400 \mathrm{~K})$. Interestingly, if water was added to the TOPO based reaction, either before or after the injection of TOPSe, the conversion of TOPSe could be increased to almost 100\% (Figure 3-1).

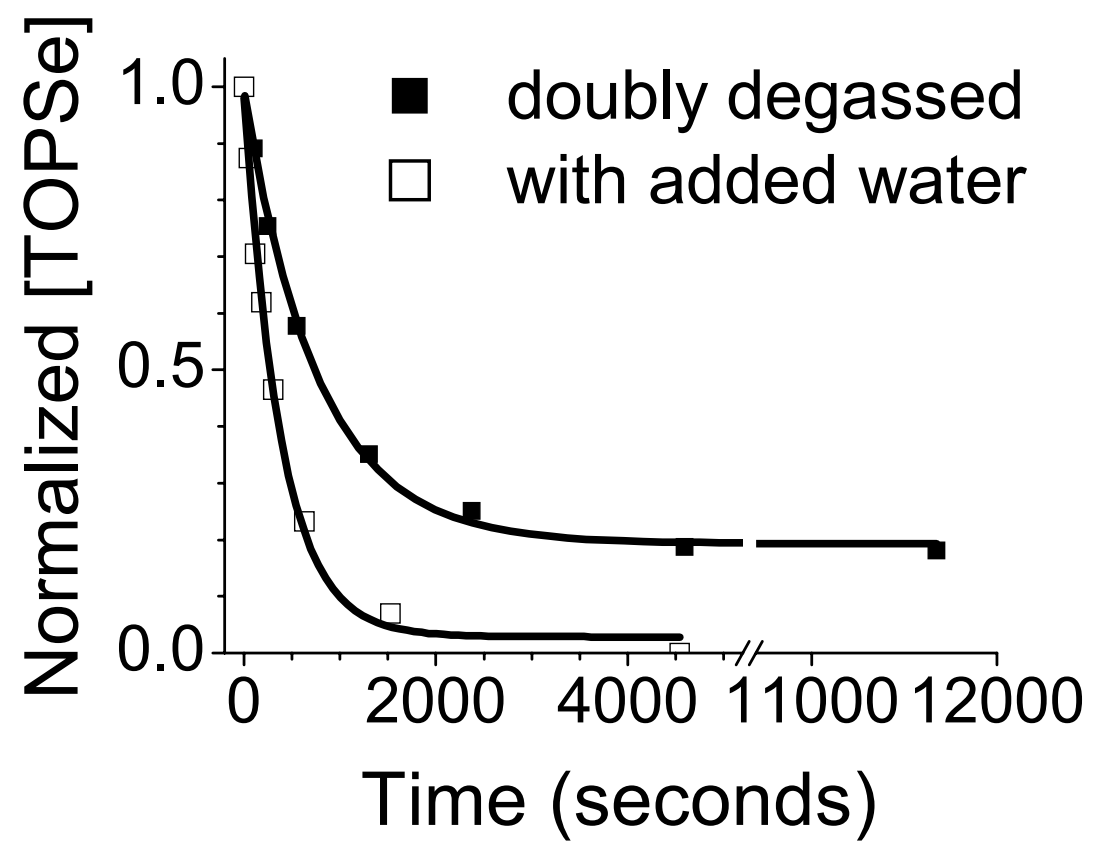

Figure 3-1. Single exponential fits to the disappearance of TOPSe in the presence of CdODPA in TOPO. Cd-ODPA and TOPSe in TOPO at $260{ }^{\circ} \mathrm{C}$. For the reactions with added water, $100 \mathrm{mg}$ of water was added to the flask at $100^{\circ} \mathrm{C}$ via a syringe after the second degassing but before TOPSe injection. 


\begin{tabular}{|c|c|c|c|c|c|c|}
\hline & & & & & onvers & \\
\hline & Material & Precursors & Solvent $\left({ }^{\circ} \mathrm{C}\right)$ & $k_{\mathrm{obs}} \times 10^{3}\left(\mathrm{~s}^{-1}\right)$ & $(\%)^{a}$ & Note \\
\hline 1 & $\mathrm{CdSe}$ & Cd-ODPA/TOPSe & TOPO (290) & $1.7 \pm 0.4$ & 84 & $b$ \\
\hline 2 & $\mathrm{CdSe}$ & Cd-ODPA/TOPSe & TOPO (260) & $1.30 \pm 0.08$ & 82 & $c$ \\
\hline 3 & $\mathrm{CdS}$ & Cd-ODPA/TOPS & TOPO (290) & $0.90 \pm 0.54$ & 30 & $d$ \\
\hline 4 & $\mathrm{CdSe}$ & Cd-OA/TOPSe & $\mathrm{C}_{9} \mathrm{D}_{20}(117)$ & $1.51 \pm 0.04$ & 65 & $e$ \\
\hline 5 & $\mathrm{CdSe}$ & Cd-OA/TBPSe & $\mathrm{C}_{10} \mathrm{D}_{22}(127)$ & $2.57 \pm 0.13$ & 68 & $e$ \\
\hline 6 & $\mathrm{CdSe}$ & $\mathrm{Cd}-\mathrm{OA} / i$-TPPSe & $\mathrm{C}_{9} \mathrm{D}_{20}(127)$ & 0 & 0 & $f$ \\
\hline 7 & $\mathrm{ZnSe}$ & Zn-ODPA/TOPSe & TOPO (290) & 0 & 0 & $g$ \\
\hline 8 & - & $\mathrm{H}_{2}$-ODPA/TOPSe & TOPO (290) & - & 15 & $h$ \\
\hline
\end{tabular}

Table 3-1. Exponential fits to the disappearance of phosphine chalcogenide. ${ }^{a}$ Conversions were calculated using either the amount of TOPSe injected and [TOPSe] of the last aliquot (TOPO based synthesis) or the fitting results (in situ studies). The reported error in $k_{\mathrm{obs}}$ is the uncertainty from the fit to a single exponential. ${ }^{b}$ Double degassed, a mixture of TOPSe $(0.70 \mathrm{~g})$ and toluene $(0.30 \mathrm{~g})$ was injected at $320{ }^{\circ} \mathrm{C} .{ }^{c}$ Double degassed. ${ }^{d}$ Double degassed, a mixture of TOPS $(0.65 \mathrm{~g})$ and toluene $(0.30 \mathrm{~g})$ was injected at $320{ }^{\circ} \mathrm{C} .{ }^{e}$ in-situ NMR monitoring. ${ }^{f}$ in-situ NMR monitoring, reaction time $=20$ min. ${ }^{g}$ Double degassed, reaction time $=100 \mathrm{~min} .{ }^{h}$ Reaction time $=4 \mathrm{hr}$. 


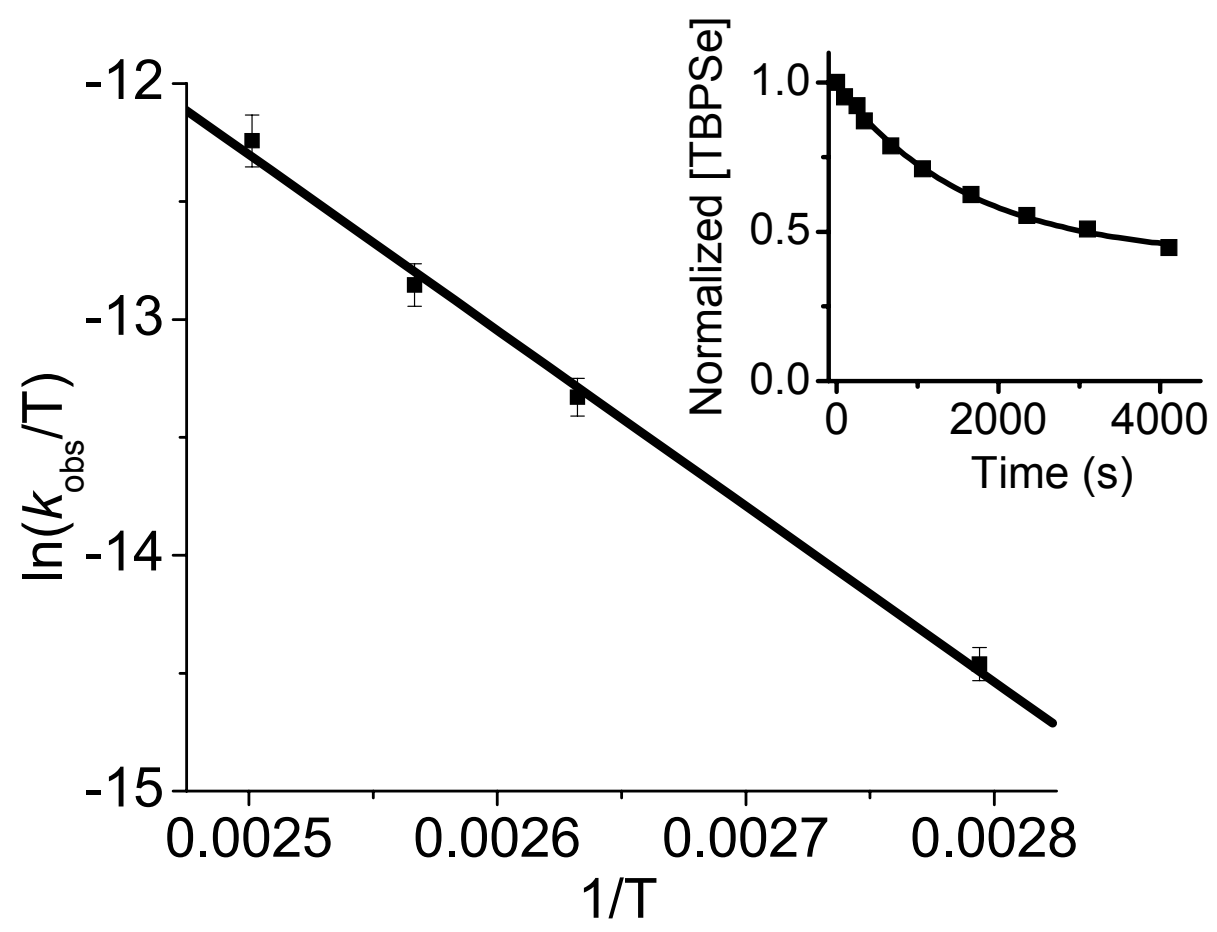

Figure 3-2. Eyring plot of TBPSe reaction with Cd-OA in $n$-decane- $d_{22}$. The reactions were carried out at $358 \mathrm{~K}, 380 \mathrm{~K}, 390 \mathrm{~K}$, and $400 \mathrm{~K}$. T: temperature (Kelvin). The inset shows a representative single exponential fit to the integrated TBPSe resonance at $380 \mathrm{~K}$. 


\begin{tabular}{cccccc}
\hline & Temp. & Cd (eq.) & OA (eq.) & TBPSe (eq.) & Decay constant (s) \\
\hline 1 & $399.8 \mathrm{~K}$ & 1 & 4 & 1 & $388 \pm 19$ \\
2 & $379.5 \mathrm{~K}$ & 1 & 4 & 1 & $1002 \pm 87$ \\
3 & $389.6 \mathrm{~K}$ & 1 & 4 & 1 & $603 \pm 27$ \\
$4^{a}$ & $389.6 \mathrm{~K}$ & 1 & 4 & 1 & $664 \pm 19$ \\
5 & $389.6 \mathrm{~K}$ & 1 & 4 & 0.5 & $911 \pm 122$ \\
6 & $389.6 \mathrm{~K}$ & 0.5 & 2 & 1 & $673 \pm 59$ \\
7 & $389.6 \mathrm{~K}$ & 0.5 & 2 & 0.5 & $1027 \pm 81$ \\
8 & $389.6 \mathrm{~K}$ & 0.5 & 4 & 1 & $581 \pm 52$ \\
9 & $389.6 \mathrm{~K}$ & 0.5 & 2 & 2 & $332 \pm 13$ \\
\hline
\end{tabular}

Table 3-2. Decay constant of TBPSe in the presence of Cd-OA and H-OA in ODE. For all the reagent concentrations, 1 eq. is $0.36 \mathrm{~mol} \cdot \mathrm{kg}^{-1}$. The amount of OA indicates the total amount of OA used in the synthesis, including that in the form of Cd-OA complex. ${ }^{a}$ : TOPSe was used instead of TBPSe. 


\begin{tabular}{cccccc}
\hline & $\mathrm{T}\left({ }^{\circ} \mathrm{C}\right)$ & $\mathrm{Cd}($ eq. $)$ & ODPA (eq.) & TOPSe (eq.) & Decay constant (s) \\
\hline 1 & 260 & 1 & 2 & 0.9 & $768 \pm 44$ \\
3 & 260 & 1 & 2 & 0.9 & $710 \pm 80$ \\
4 & 260 & 1 & 2 & 1.7 & $809 \pm 66$ \\
5 & 260 & 1 & 4 & 0.9 & $1213 \pm 148$ \\
6 & 260 & 0.5 & 2 & 0.9 & $360 \pm 58$ \\
$7^{a}$ & 260 & 1 & 2 & 0.9 & $338 \pm 47$ \\
$8^{b}$ & 260 & 1 & 2 & 0.9 & $393 \pm 37$ \\
$9^{c}$ & 260 & 0.5 & 2 & 0.9 & $382 \pm 26$ \\
10 & 270 & 1 & 2 & 0.9 & $789 \pm 79$ \\
$11^{d}$ & 260 & 1 & 2 & 0.9 & $671 \pm 72$ \\
$12^{e}$ & 260 & 1 & 2 & 0.9 & $335 \pm 35$ \\
\hline
\end{tabular}

Table 3-3. Decay constants of TOPSe in the presence of Cd-ODPA and TOPO. For all the reagent concentrations, 1 eq. is $1.6 \mathrm{~mol} \cdot \mathrm{kg}^{-1}$. The amount of ODPA indicates the total amount of ODPA used in the synthesis, including that in the form of Cd-ODPA complex. ${ }^{a}: 108 \mathrm{mg}$ of water was added; ${ }^{b}: 94 \mathrm{mg}$ of water was added; ${ }^{c}: 0.5 \mathrm{eq} \mathrm{ZnO}$ and $0.5 \mathrm{eq} \mathrm{CdO}$ was used; ${ }^{d}$ : Use $\mathrm{Cd}(\mathrm{Me})_{2} ;{ }^{e}: 0.5$ eq $\mathrm{P}_{2} \mathrm{O}_{5}$ was added. 


\subsection{Mechanism of Precursor Decomposition and Monomer Formation}

The formation of acid anhydride and dependence of reaction kinetics on the surfactant concentration suggest that phosphonic and carboxylic acids are also reactants responsible for the conversion of precursor molecules to the inorganic materials. In particular, the conversion of TOPSe and TBPSe to their corresponding phosphine oxides is linked to the formation of anhydrides of $n$-octadecylphosphonic acid and oleic acid suggesting that phosphonic and carboxylic acids are responsible for cleavage of the phosphorus - chalcogen double bond. A partial mechanism for this transformation is shown below in Scheme 3-1.

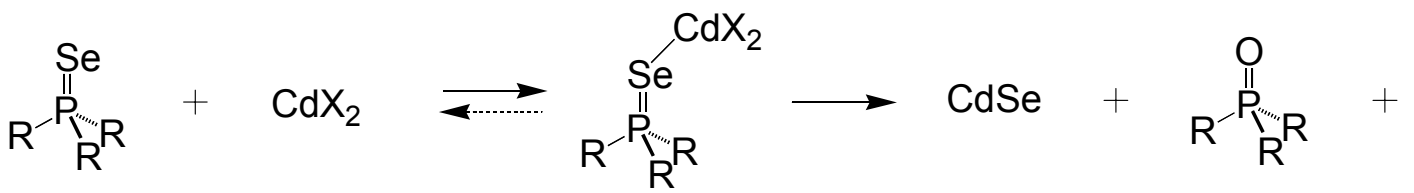

$$
\begin{aligned}
& X=-{ }^{-} \stackrel{\stackrel{O}{C}}{-} C_{C_{17}} H_{33}
\end{aligned}
$$

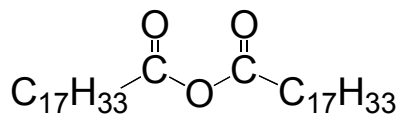

$$
\begin{aligned}
& X=\stackrel{\text { OII }}{\mathrm{O}}-\stackrel{\mathrm{O}}{\mathrm{O}}-\mathrm{C}_{18} \mathrm{H}_{37}
\end{aligned}
$$

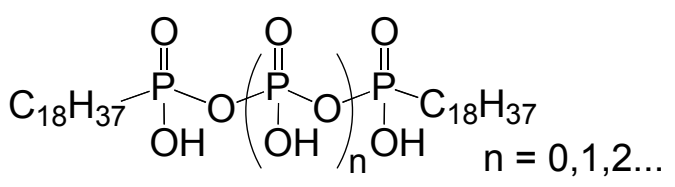

Scheme 3-1. Proposed reaction pathway for precursor conversion.

TOPSe-C $\mathbf{d}^{2+}$ coordination. Alkyl and aryl phosphine chalcogenides have been shown to coordinate to a large number of transition metal ions, including $\mathrm{Cd}^{2+}$ and $\mathrm{Zn}^{2+}$, resulting in a weakening of the $\mathrm{P}=\mathrm{E}(\mathrm{E}=\mathrm{S}, \mathrm{Se}, \mathrm{Te})$ double bond. ${ }^{4-6}$ Our ${ }^{31} \mathrm{P}$ NMR studies in Chapter 2 show that the resonance of TOPSe shifts to higher ppm value along with a decreased ${ }^{1} J^{31} \mathrm{P}_{-}{ }^{77} \mathrm{Se}$ in $\mathrm{CDCl}_{3}$ solution of cadmium 2-ethylhexanoate, suggesting the 
formation of a TOPSe- $\mathrm{Cd}^{2+}$ complex. ${ }^{7-9}$ These spectroscopic changes were not observed, however, when TOPSe was combined with Cd-ODPA supporting a weaker coordination of TOPSe to Cd-ODPA than Cd-OA. This difference may be due, in part, to a more coordinatively unsaturated cadmium center in Cd-OA and offers an explanation of the faster TOPE cleavage by Cd-OA.

Cleavage of the $\mathbf{P}=\mathbf{E}(\mathrm{E}=\mathrm{S}, \mathrm{Se}, \mathrm{Te})$ bond. A number of experimental observations suggest that the TOPE precursor is converted to cadmium and zinc chalcogenides by a substitution reaction in which a Lewis acidic cadmium or zinc center activates TOPE to nucleophilic attack by phosphonate or carboxylate. First, TOPSe cleavage proceeds at a rate two orders of magnitude faster in the presence of Cd-ODPA and TOPO than $\mathrm{H}_{2}$-ODPA and TOPO alone, providing support for the Lewis acid activation step. Second, the large and negative entropy of activation extrapolated from the Eyring plot in Figure 3-2 suggests that the steps leading to TBPSe cleavage in the presence of the Lewis acidic cadmium center involves the association of two or more molecules. This suggestion is made tentatively, however, since the single exponential fits to TOPSe decay are not likely to result from first order kinetics. This is especially true because the reactions approach partial conversion and were not run under pseudo first order conditions. Third, the sterically hindered $i$-TPPSe reacts with Cd-OA much slower than does TBPSe which argues against unimolecular decomposition of the $\mathrm{P}=\mathrm{Se}$ fragment.

Finally formation of phosphine oxides via cleavage of $\mathrm{P}=\mathrm{X}$ double bonds frequently involves two steps where an electrophile is first bound to $\mathrm{X}$ followed by addition of an oxygen nucleophile to the phosphorus center. Previous studies of alkyl and 
aryl phosphine selenides and sulfides $(\mathrm{X}=\mathrm{Se}, \mathrm{S})$ have shown that conversion to the corresponding oxide under non-oxidizing conditions can proceed via a Lewis acid catalyzed substitution mechanism. In these studies triphenylphosphine selenide or sulfide, when activated by trifluoacetic anhydride, undergoes attack by nucleophiles as weak as trifluoroacetate yielding triphenylphosphine oxide. ${ }^{10,11}$ In a similar vein, the accepted mechanisms for the cleavage of Wittig $\left(X=\mathrm{CR}_{2}\right)$ and aza-Wittig $(\mathrm{X}=\mathrm{NR})$ reagents all involve addition to the phosphorus center upon reaction of an electrophile with the nucleophilic terminus of the $\mathrm{P}=\mathrm{X}$ double bond. ${ }^{12}$ All these points argue for a similar cleavage reaction under our conditions where nucleophilic addition to the phosphorus center proceeds upon Lewis acid activation of TOPE by the cadmium or zinc precursor.

Based on the above arguments, a hypothetical transition structure of the $\mathrm{TOP}=\mathrm{E}$ cleavage is shown in Figure 3-3. During this process, the phosphorous atom is attacked by an incoming nucleophile $\mathrm{X}$, weakening the $\mathrm{P}-\mathrm{X}$ bond and partially breaking the $\mathrm{P}=\mathrm{E}$ bond. Quantum chemical calculation (see next chapter) suggests that this nucleophilic attack is more likely to be an intramolecular reaction rather than an intermolecular one, due to the high energy penalty of forming charged species in the common solvents used in nanocrystal synthesis.

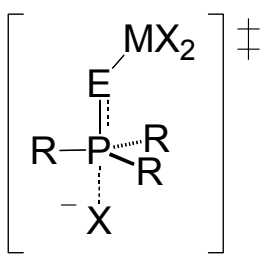

Figure 3-3 Hypothetical transition structure for TOPE cleavage. 
Finally, our results argue against other proposals for the mechanism of ME $(\mathrm{M}=\mathrm{Cd}, \mathrm{Zn}, \mathrm{E}=\mathrm{S}, \mathrm{Se}, \mathrm{Te})$ nanocrystals formation from tri-alkylphosphine chalcogenides and phosphonic and oleic acid complexes of cadmium and zinc. In particular, pathways involving formation of atomic cadmium, zinc or chalcogen do not adequately explain our results. These reactions can be expected to display positive entropies of activation since two or more molecules should be generated in the homolysis reaction. Neither do such pathways easily explain the clean formation of phosphonic acid anhydrides or oleic acid anhydride and tri-alkylphosphine oxide observed in this study.

\subsection{Implications to the Synthesis of Group II-VI Nanocrystals}

Reactivity of phosphine chalcogenides can be readily predicted from the dissociation energy $(D)$ of the $\mathrm{P}=\mathrm{X}$ bond and the steric hindrance of the organic skeleton. For the precursors that only differ in the chalcogen atom, the reactivity is controlled by the $D$ of the $\mathrm{P}=\mathrm{X}$ bond, which is known to follow the trend: $\mathrm{P}=\mathrm{S}>\mathrm{P}=\mathrm{Se}>\mathrm{P}=\mathrm{Te}$. Based on this information, we can readily predict that for different TOPX, the relative rate of reaction will be TOPTe $>$ TOPSe $>$ TOPS. This trend has been verified for TOPSe and TOPS in the current study by measuring their decay kinetics in the presence of Cd-ODPA in TOPO. Reaction kinetics of TOPTe was not measured in the current study. TOPTe reacts with oxygen rapidly at room temperature, which makes the experiment more

difficult. Preliminary results based on the rate of color change did suggest that the reaction kinetics of TOPTe was faster than that of TOPSe, which was also consistent with previous observation. ${ }^{13}$ 
For the precursors that only differ in the organic skeleton, the relative reaction rate is controlled by the steric hindrance near the phosphorous atom. It is well known that in a $S_{N} 2$ reaction, the reaction rate will decrease with increasing steric hindrance near the reaction center. This effect is best demonstrated by comparing the reactivity of $i$-TPPSe and TBPSe in the presence of Cd-OA (Table 3-1). No reaction was observed between $i$ TPPSe and Cd-OA at $127^{\circ} \mathrm{C}$ within 20 min while under the same reaction condition, most of TBPSe was consumed. It was also shown recently that TBPTe, which has three butyl groups on phosphorous, is more reactive than TOPTe, which has three relatively larger octyl groups. ${ }^{14}$

Metal precursor. The nature of metal precursor also affects the reaction rate, since the reaction is 'catalyzed' by the metal ion in the first binding step. The Lewis acidity of the metal ion determines its 'catalytic' efficiency. For example, TOPSe uses its Se atom, which is a soft base, to bind $\mathrm{Cd}^{2+}$. One can thus predict that $\mathrm{Zn}^{2+}$, a harder Lewis acid than $\mathrm{Cd}^{2+}$, will bind to TOPSe weaker than $\mathrm{Cd}^{2+}$ does. In addition, $\mathrm{Zn}^{2+}$ also binds to the oxygen atom of $\mathrm{H}_{2}$-ODPA and $\mathrm{H}-\mathrm{OA}$, a strong Lewis base, stronger than $\mathrm{Cd}^{2+}$ does. As a result, the reaction rate of $\mathrm{ZnSe}$ nanocrystal synthesis should be slower than that of CdSe nanocrystal synthesis. This prediction is indeed observed in the present work. Under the same reaction condition, the reactivity of Zn-ODPA complex toward TOPSe is much lower than that of Cd-ODPA (Table 3-1). Using the 'doubly degassed' protocol, we did not observe any change in the ${ }^{31} \mathrm{P}$ NMR spectrum after heating the reaction at $290{ }^{\circ} \mathrm{C}$ for over $100 \mathrm{~min}$. However, the formation of $\mathrm{ZnSe}$ nanocrystals is observed, although in very low yield, as determined by transmission electron microscopy and UV-Vis absorption spectroscopy. 
The role of surfactant as a reagent. Surfactants like $\mathrm{H}_{2}$-ODPA and $\mathrm{H}-\mathrm{OA}$ have been extensively used to control the growth of nanocrystals. Their major role has long been believed to be selective adhesion to the nanocrystal surface, ${ }^{1,15}$ as well as controlling monomer solubility. ${ }^{2,3,16}$ However, our results show that $\mathrm{H}_{2}$-ODPA and H-OA are also reactants in the TOPSe cleavage. Hence changing the concentration of these surfactants ${ }^{1,15,17}$ will likely change the TOPE cleavage kinetics in addition to the binding of surfactants to the nanocrystal surface. This is especially important given that the alkylphosphine chalcogenide cleavage is likely the reaction leading to the formation of semiconductor monomers and hence the rate of this cleavage will influence particle nucleation and growth.

Further, as the TOPSe cleavage reaction proceeds, the $\mathrm{H}_{2}$-ODPA and H-OA surfactants are converted to their corresponding anhydrides and selenium is liberated into the solution, which can both affect the identity of the reactive cadmium complex. These changes to the cadmium precursor and surfactant molecules undoubtedly impact the mechanistic details of the TOPE binding and cleavage further limiting the interpretation of the reaction kinetics and yield. Several of our observations highlight this point. That added water leads to an increase in the TOPSe conversion suggests that cadmium coordinated by $\mathrm{H}_{2}$-ODPA is more reactive than when coordinated by the anhydride of ODPA. ${ }^{18,19}$ This does not, however, explain the low conversion observed in the CdSe synthesis using Cd-OA and TBPSe in a non-coordinating solvent, since $(\mathrm{OA})_{2} \mathrm{O}$ should bind cadmium only weakly. The limited yield in this case may arise from the build up of dissolved monomers which compete with TOPSe for binding to Cd-OA. Additionally, the 
reactivity of $\mathrm{Cd}(\mathrm{OA})_{2}$ may be decreased by coordination of the phosphine oxide product. $^{6,20}$

Solvent can affect the reaction kinetics and conversion in several ways. The polarity of the solvent determines the amount of water in the reaction mixture and thus changing the overall conversion at long reaction time. The solvent polarity also affects the relative free energy of the substrates and transition states, thus changing the activation free energy. A more complicated scenario arises when free phosphine was used in the synthesis as a co-solvent. Phosphine sulfide, selenide, and especially telluride have been shown to undergo chalcogen exchange with free phosphine. ${ }^{21}$

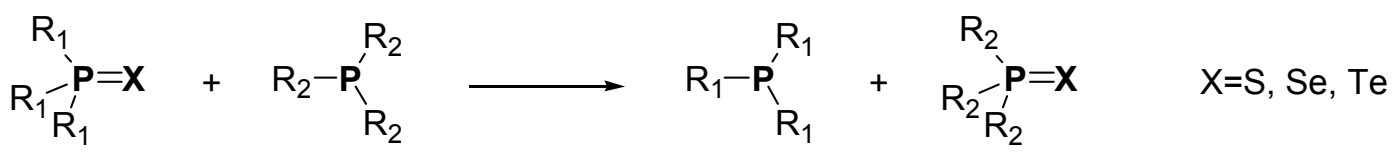

Since the substitution on phosphorus is expected to affect the reaction rate, redistribution of chalcogen atom to a different phosphine will change the overall reaction kinetics. On the other hand, this also points to a possible way to further manipulate the reaction rate.

Water content in the reaction mixture significantly changes the reaction kinetics. The amount of water in the reaction highly depends on the detailed experimental procedure. For example, aging of $\mathrm{Cd}$ precursor has been shown to slow the growth rate and increase the aspect ratio of nanorods. ${ }^{22}$ We found that condensed water droplets formed during the $\mathrm{CdO}$ decomposition evaporate completely under $\mathrm{Ar}$ flow within two days. The reported change in the reaction kinetics and aspect ratio of the nanorod could potentially be due to the effect of removing water. 


\section{References}

(1) Peng, Z. A.; Peng, X. G. J. Am. Chem. Soc. 2001, 123, 1389-1395.

(2) van Embden, J.; Mulvaney, P. Langmuir 2005, 21, 10226-10233.

(3) Bullen, C. R.; Mulvaney, P. Nano Lett. 2004, 4, 2303-2307.

(4) Karayannis, N. M.; Mikulski, C. M.; Pytleweski, L. L. Inorg. Chim. Acta Rev. 1971, 5, 69-105.

(5) Lobana, T. S.; Gupta, T. R.; Sandhu, S. S. Polyhedron 1982, 1, 781-783.

(6) Lobana, T. S. In The chemistry of organophosphorous compounds; Hartley, F. R., Ed.; John Wiley \& Sons: 1992; Vol. 2, p 487.

(7) Grim, S. O.; Walton, E. D.; Satek, L. C. Can. J. Chem. 1980, 58, 14761479.

(8) Dean, P. A. W.; Polensek, L. Can. J. Chem. 1980, 58, 1627-1632.

(9) Dean, P. A. W.; Hughes, M. K. Can. J. Chem. 1980, 58, 180-190.

(10) Chen, C. H.; Brighty, K. E. Tetrahedron Lett. 1980, 21, 4421-4424.

(11) Helinski, J.; Skrzypczynski, Z.; Wasiak, J.; Michalski, J. Tetrahedron Lett. $1990,31,4081-4084$.

(12) Smith, M. B.; March, J.; March's Advanced Organic Chemistry: Reactions, Mechanisms, and Structure. 5th Edition ed.; Wiley Interscience: New York, 2001, p 1231.

(13) Bailey, R. E.; Nie, S. M. J. Am. Chem. Soc. 2003, 125, 7100-7106.

(14) Yu, W. W.; Wang, Y. A.; Peng, X. G. Chem. Mater. 2003, 15, 4300-4308.

(15) Manna, L.; Scher, E. C.; Alivisatos, A. P. J. Am. Chem. Soc. 2000, 122, $12700-12706$. 
(16) Yu, W. W.; Peng, X. G. Angew. Chem. Int. Ed. 2002, 41, 2368-2371.

(17) Manna, L.; Milliron, D. J.; Meisel, A.; Scher, E. C.; Alivisatos, A. P. Nature Mater. 2003, 2, 382-385.

(18) Spanhel, L.; Haase, M.; Weller, H.; Henglein, A. J. Am. Chem. Soc. 1987, $109,5649-5655$.

(19) We note that a cadmium center coordinated by the more acidic anhydride of ODPA is likely to bind and activate TOPSe to a greater extent, but may also be a weaker nucleophile in the cleavage step.

(20) Bond, A. M.; Colton, R.; Ebner, J.; Ellis, S. R. Inorg. Chem. 1989, 28, 4509-4516.

(21) Brown, D. H.; Cross, R. J.; Keat, R. J. Chem. Soc., Dalton Trans. 1980 , 871-874.

(22) Peng, Z. A.; Peng, X. G. J. Am. Chem. Soc. 2002, 124, 3343-3353. 


\section{Chapter 4}

\section{Density Functional Theory Study of the Precursor Evolution in the Synthesis of CdSe Nanocrystals}

\subsection{Introduction}

In the previous chapters, I have presented an experimental study on the reactions between the precursors in the synthesis of CdSe and other group II-VI nanocrystals. A mechanism was proposed for the decomposition of the precursors and formation of monomer. However, experimental results only provided limited information on the detailed reaction pathway. Especially, direct characterization of monomer in the growth solution has not been possible due to the technical difficulties associated with detecting this unstable, reactive intermediate. To the best of our knowledge, the structure of the monomer and the detailed reaction pathway of its generation have not been discussed in the literature.

In this chapter, I use density functional theory (DFT) calculation to study the detailed reaction pathway of precursor decomposition and monomer formation in the synthesis of CdSe nanocrystals. The particular system I choose to study is the synthesis of CdSe nanocrystal using phosphine selenide and cadmium carboxylate in a non-polar solvent (e.g., $n$-octadecene, or ODE) using a carboxylic acid as surfactant. This synthesis has been widely used in the synthesis of cadmium and zinc chalcogenides nanocrystals. ${ }^{1}$ 
Compared to the CdSe nanocrystal synthesis in TOPO using phosphonic acid surfactant, the chemistry of this ODE/carboxylic acid based synthesis is simpler and better characterized.

The calculations show that phosphine selenide binds to cadmium carboxylate using its selenium atom to give a $\left(\mathrm{R}_{3}^{2} \mathrm{P}=\mathrm{Se}\right) \mathrm{Cd}\left(\mathrm{R}^{1} \mathrm{COO}\right)_{2}\left(\mathrm{R}^{1} \mathrm{COOH}\right)_{\mathrm{x}}(\mathrm{x}=0,1)$ complex. The phosphine selenide in this complex is attacked by a carboxylate ligand of the same complex in an intramolecular $\mathrm{SN}_{2}$ reaction to give a pentavalent phosphorous intermediate. The selenium atom of this intermediate accepts a proton from a carboxylic acid ligand, breaking the P-Se bond and eventually forming a $\mathrm{Cd}(\mathrm{SeH})\left(\mathrm{R}^{1} \mathrm{COO}\right)$ complex, phosphine oxide, and carboxylic acid anhydride. We suggest that this $\mathrm{Cd}(\mathrm{SeH})\left(\mathrm{R}^{1} \mathrm{COO}\right)$ complex is the possible monomer for the growth of CdSe nanocrystal.

\subsection{Theoretical Method}

Unless otherwise specified, all calculations were carried out using pcGAMESS program $^{2}$ at the $\mathrm{B}^{2} \mathrm{LYP}^{3-6}$ (using VWN5 functional) level of theory. The following basis $\operatorname{sets}^{7}$ (spherical harmonic functions) were used: SDB-ccPVTZ basis in conjunction with Stuttgart-Dresden-Bonn relativistic effective core potential (ECP) for Cd ((8s7p6d2f1g) $\rightarrow[6 \mathrm{~s} 5 \mathrm{p} 3 \mathrm{~d} 2 \mathrm{f} 1 \mathrm{~g}])$ and $\mathrm{Se}((14 \mathrm{~s} 10 \mathrm{p} 2 \mathrm{~d} 1 \mathrm{f}) \rightarrow[3 \mathrm{~s} 3 \mathrm{p} 2 \mathrm{~d} 1 \mathrm{f}]){ }^{8}$ cc-PVTZ basis for P $((15 \mathrm{~s} 9 \mathrm{p} 2 \mathrm{~d} 1 \mathrm{f}) \rightarrow[5 \mathrm{~s} 4 \mathrm{p} 2 \mathrm{~d} 1 \mathrm{f}]) ;^{9}$ and $6-31 \mathrm{G}^{*}+$ basis for $\mathrm{O}, \mathrm{C}$, and $\mathrm{H}^{10}{ }^{10}$ The molecular geometries were relaxed in vacuum without constrain until the maximum energy gradient is smaller than $5 \times 10^{-5}$ hartree/bohr. Frequency calculation was performed at the same level of theory to confirm that the obtained geometry is a minima or transition state, as appropriate. Thermodynamic properties were calculated at $300 \mathrm{~K}$ assuming rigid rotor 
model and ideal gas behavior. Intrinsic reaction coordinate (IRC) calculations were performed to ensure that the transition states structure connects two concerned minima. The electrostatic solvation effect was described by polarization continuum model (PCM) using $n$-heptane as the solvent. The geometry optimized in vacuum was used in a single point PCM energy calculation. The reported thermodynamic quantities were obtained in vacuum unless otherwise specified.

\subsection{Results and Discussion}

Verification of computational method. Extensive calculations were carried out to ensure that our choice of computational method could accurately reproduce the geometry and energy of the modeled chemistry. The reliability of the calculated geometry was checked by comparing optimized structure of tri-methyl-phosphine selenide and a phosphine selenide - cadmium complex $\left(\mathrm{Cd}[\mathrm{R} 2 \mathrm{P}(\mathrm{Se}) \mathrm{C}(\mathrm{S}) \mathrm{NPh}]_{2}, \quad \mathrm{R}=\mathrm{CH}_{3}\right)$ with the experimental results. Within B3LYP level of theory, we found that the popular Lanl2dz ECP and basis ${ }^{11-13}$ for $\mathrm{Cd}, \mathrm{Se}$, and $\mathrm{P}$ atoms could not accurately reproduce the experimental observed geometries though addition of polarization and diffusion functions (Lanl2dzdp ${ }^{14}$ basis for Se and P atoms) significantly improves the result. Use of cc-PVTZ quality of basis sets for the post-second-row heavy atoms (SDB-ccPVTZ for Cd and Se, cc-PVZT for P) is necessary to reproduce the structure of the two model compounds. The quality of the energy calculation was checked by computing the homolytic Cd-Se bond dissociation energy (BDE) of CdSe molecule in gas phase. The BDE calculated using B3LYP/SDB-cc-PVTZ method slightly underestimate $\left(\sim 2-6 \mathrm{kcal} \cdot \mathrm{mol}^{-1}\right)$ the highly accurate CCSD(T)/aug-cc-PVnZ-PP ( $\mathrm{n}=\mathrm{T}, \mathrm{Q})^{15}$ result. 


\begin{tabular}{lllll}
\hline Basis & $\begin{array}{l}\mathrm{P}=\text { Se Bond } \\
(\text { error} \%)\end{array}$ & $\begin{array}{l}\text { P-C Bond } \\
(\text { error\% })\end{array}$ & $\begin{array}{l}\angle \mathrm{SePC} \\
(\text { error\%) }\end{array}$ & $\begin{array}{l}\angle \mathrm{CPC} \\
(\text { error\%) }\end{array}$ \\
\hline Lanl2dz $^{\mathrm{a}}$ & $2.267 \AA$ & $1.873 \AA$ & $113.9^{\circ}$ & $104.7^{\circ}$ \\
Lanl2dzdp $^{\mathrm{b}}$ & $2.147 \AA$ & $1.852 \AA$ & $114.4^{\circ}$ & $104.2^{\circ}$ \\
cc-PVTZ $^{\mathrm{c}}$ & $2.120 \AA$ & $1.835 \AA$ & $114.5^{\circ}$ & $104.0^{\circ}$ \\
Exp & $2.111 \AA$ & $1.786 \AA$ & $113.1^{\circ}$ & $105.7^{\circ}$ \\
\hline
\end{tabular}

Table 4-1. B3LYP Optimized structure of tri-methylphophine selenide. ${ }^{\text {a: }}$ Lanl2dz for Se and P, 6-31G*+ for O, C, and H. ${ }^{\mathrm{b}}$ : Lanl2dzdp for Se and P, 6-31G*+ for O, C, and H. ${ }^{\mathrm{c}}$ : SDB-ccPVTZ for Se, ccPVTZ for P, 6-31G*+ for O, C, and H. Experimental value are obtained from x-ray diffraction. ${ }^{16}$

\begin{tabular}{|c|c|c|c|c|c|c|}
\hline \multicolumn{4}{|c|}{ Basis } & \multirow{2}{*}{ Cd-Se $(\AA)$} & \multirow{2}{*}{$\mathrm{Se}=\mathrm{P}(\AA)$} & \multirow{2}{*}{ Cd-S $(\AA)$} \\
\hline $\mathrm{Cd}$ & $\mathrm{Se}$ & $\mathrm{P}$ & $S$ & & & \\
\hline Lanl2dz & Lanl2dzdp & Lanl2dzdp & Lanl2dzdp & 2.759 & 2.207 & 2.629 \\
\hline $\begin{array}{l}\text { SDB- } \\
\text { ccpvtz }\end{array}$ & $\begin{array}{l}\text { SDB- } \\
\text { ccpvtz }\end{array}$ & Lanl2dzdp & cc-PVTZ & 2.695 & 2.194 & 2.554 \\
\hline $\begin{array}{l}\text { SDB- } \\
\text { ccpvtz }\end{array}$ & $\begin{array}{l}\text { SDB- } \\
\text { ccpvtz }\end{array}$ & cc-PVTZ & cc-PVTZ & 2.694 & 2.182 & 2.551 \\
\hline \multicolumn{4}{|c|}{ Exp } & $\begin{array}{l}2.613- \\
2.625\end{array}$ & $\begin{array}{l}2.145- \\
2.152\end{array}$ & $\begin{array}{l}2.488- \\
2.523\end{array}$ \\
\hline
\end{tabular}


Table 4-2. Selected bond length of optimized structure of $\mathrm{Cd}\left[\mathrm{R}_{2} \mathrm{P}(\mathrm{Se}) \mathrm{C}(\mathrm{S}) \mathrm{NPh}\right]_{2}$. 6$31+\mathrm{G}^{*}$ basis was used for $\mathrm{O}, \mathrm{N}, \mathrm{C}$, and $\mathrm{H}$. R was set to methyl group to save computational time while the experimental value ${ }^{17}$ was reported for $\mathrm{R}=$ cyclohexyl.

\begin{tabular}{lll}
\hline Method & Singlet CdSe BDE & Triplet CdSe BDE \\
\hline B3LYP/SDB-ccPVTZ & 13.22 & 13.75 \\
CCSD(T)/aug-ccPVTZ-PP & 15.70 & 12.76 \\
CCSD(T)/aug-ccPVQZ-PP & 19.0 & 14.89 \\
\hline
\end{tabular}

Table 4-3. Calculated bond dissociation energy (BDE) of CdSe molecule $\left(\mathrm{kcal} \cdot \mathrm{mol}^{-1}\right)$. The BDE was corrected for BSSE, but not for zero point vibrational energy (ZPE).

\section{General comment on the theoretical modeling of CdSe nanocrystal synthesis} using $\mathbf{C d}\left(\mathbf{R}^{1} \mathbf{C O O}\right)_{2}$ and $\mathbf{R}^{2}{ }_{3} \mathbf{P}=$ Se. In a typical synthesis of CdSe nanocrystal, cadmium oleate $\left(\mathrm{Cd}\left(\mathrm{R}^{1} \mathrm{COO}\right)_{2}, \mathrm{R}^{1}=\mathrm{Oleyl}\right)$ and tri- $n$-butylphosphine selenide $\left(\mathrm{R}_{3}^{2} \mathrm{P}=\mathrm{Se}, \mathrm{R}^{2}=n\right.$-butyl $)$ was heated in a mixture of non-polar solvent (e.g., ODE) and oleic acid. Several key mechanistic features of this reaction have been documented in the previous chapters. ${ }^{18}$ First, the final reaction products are CdSe nanocrystals, phosphine oxide, and anhydride of the surfactant ligand. A balanced reaction is shown in Eq. (4-1).

$$
\mathrm{Cd}\left(\mathrm{R}^{1} \mathrm{COO}\right)_{2}+\mathrm{R}_{3}^{2} \mathrm{P}=\mathrm{Se} \rightarrow \mathrm{CdSe}+\mathrm{R}_{3}^{2} \mathrm{P}=\mathrm{O}+\left(\mathrm{R}^{1} \mathrm{COO}\right)_{2} \quad \mathrm{R}^{1}, \mathrm{R}^{2}=\text { alkyl }
$$

Second, the reaction involves $\mathrm{R}_{3}^{2} \mathrm{P}=\mathrm{Se}$ binding to $\mathrm{Cd}\left(\mathrm{R}^{1} \mathrm{COO}\right)_{2}$ using the Se atom. This binding event is likely followed by a nucleophilic attack on the phosphorous atom by a carboxylate ligand. Increasing steric hindrance near the phosphorous atom greatly 
decreases the reactivity of phosphine selenide. Third, no intermediate was detected when the reaction was monitored in situ using ${ }^{31} \mathrm{P}$ NMR spectroscopy in the range of $\sim 360 \mathrm{~K}$ to $400 \mathrm{~K} .{ }^{19}$ Finally, the reaction exhibits a large activation entropy $\left(\Delta \mathrm{S}^{\neq}=-(38 \pm 2) \mathrm{cal} \cdot \mathrm{mol}^{-}\right.$ ${ }^{1} \cdot \mathrm{K}^{-1}$, in the temperature range of $380 \mathrm{~K}$ to $400 \mathrm{~K}$ ). Based on these observations, the following reaction mechanism (scheme 4-1) was proposed to explain the formation of CdSe nanocrystals and other reaction products.

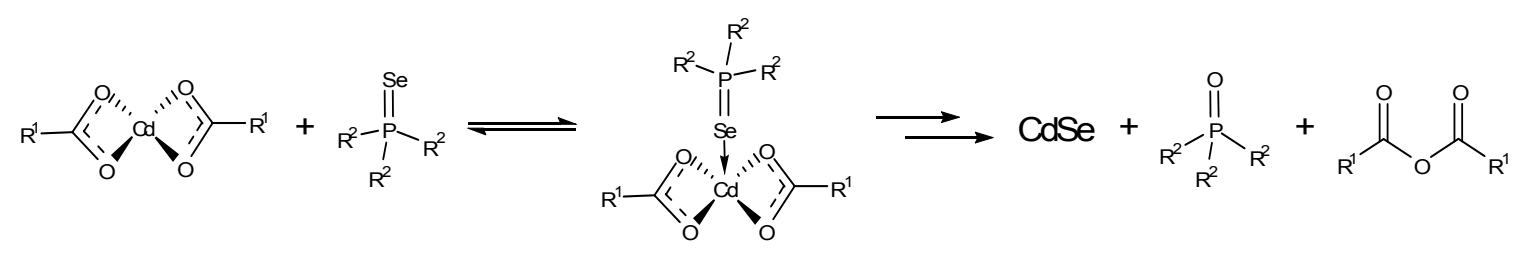

Scheme 4-1. Proposed reaction mechanism of CdSe nanocrystal synthesis from $\mathrm{Cd}(\mathrm{OA})_{2}$ and phosphine selenide. $\mathrm{R}^{1}=$ oleyl and $\mathrm{R}^{2}=$ butyl.

In this mechanism, phosphine selenide molecule is first activated by coordination to a Lewis acidic cadmium center. The activated phosphine selenide is then attacked by a nucleophile, most likely a carboxylate or a carboxylic acid. This nucleophilic attacked and its subsequent reactions eventually break the $\mathrm{P}=\mathrm{Se}$ bond and presumably generate the 'monomer'.

In order to model this complex reaction at the ab initio level, we have adapted the following simplifications. First, to save computational time, long alkyl chains were substituted with hydrogen atoms $\left(\mathrm{R}^{1}=\mathrm{R}^{2}=\mathrm{H}\right)$. Though this is a standard practice in quantum simulation, it is important to note that replacing long alkyl groups with $\mathrm{H}$ atoms reduces the steric hindrance. The change in the steric hindrance effect is especially large 
near the phosphorous atom. Second, we have decided not to consider solvation effect. This simplification should be justified since the reaction was run in a hydrocarbon solvent that has very low dielectric constant. To verify this assumption, the electrostatic solvation energy was calculated for the reactants and a number of intermidates using the geometry optimized in vacuum. The calculated solvation energies are mostly around $5 \pm 2$ $\mathrm{kcal} \cdot \mathrm{mol}-1$. As a result, the relative free energy landscape of the reaction was not significantly changed by omission of the solvation effect. Finally, we choose to consider only the monomeric form of cadmium carboxylate $\left(\mathrm{Cd}\left(\mathrm{R}^{1} \mathrm{COO}\right)_{2}\right)$ as the cadmium precursor. In reality, multinuclear complex in the form of $\mathrm{Cd}_{\mathrm{x}}\left(\mathrm{R}^{1} \mathrm{COO}\right)_{2 \mathrm{x}}\left(\mathrm{R}^{1} \mathrm{COOH}\right)_{\mathrm{y}}$ $(x>1, y>0)$ could exist, especially at low temperature. However, this should not limit the interpretation of our results too much since most CdSe synthesis was carried out at very high temperature, under which condition monomeric form of cadmium carboxylate dominates due to its large entropy. For example, within B3LYP level of theory, $\Delta G$ of reaction (4-2) is negative at $\mathrm{T}>475 \mathrm{~K}$. In comparison, most CdSe syntheses were carried out at about $573 \mathrm{~K}$.

$$
\mathrm{Cd}_{2}\left(\mathrm{R}^{1} \mathrm{COO}\right)_{4}\left(\mathrm{R}^{1} \mathrm{COOH}\right)_{2} \rightarrow \mathrm{Cd}\left(\mathrm{R}^{1} \mathrm{COO}\right)_{2}+2 \mathrm{R}^{1} \mathrm{COOH} \quad \mathrm{R}^{1}=\mathrm{H}
$$

Activation of phosphine selenide by binding to $\mathbf{C d}\left(\mathrm{R}^{\mathbf{1}} \mathbf{C O O}\right)_{2} \cdot{ }^{31} \mathrm{P} \mathrm{NMR}$ resonance of $\mathrm{R}_{3}^{2} \mathrm{P}=\mathrm{Se}\left(\mathrm{R}^{2}=n\right.$-octyl) shifts to higher $\delta$ value in the presence of cadmium carboxylate. This change in the $\mathrm{R}_{3}^{2} \mathrm{P}=\mathrm{Se}$ chemical shift suggests that $\mathrm{R}_{3}^{2} \mathrm{P}=\mathrm{Se}$ bind to cadmium carboxylate with the Se atom. It was previously suggested that this binding event activates $\mathrm{R}_{3}^{2} \mathrm{P}=\mathrm{Se}$ for the subsequent nucleophilic attack by carboxylate.

Our DFT calculation shows that $\mathrm{R}_{3}^{2} \mathrm{P}=\mathrm{Se}$ and $\mathrm{R}^{1} \mathrm{COOH}$ surfactant both bind to $\mathrm{Cd}\left(\mathrm{R}^{1} \mathrm{COO}\right)_{2}$ at $298 \mathrm{~K}$. $\mathrm{Cd}\left(\mathrm{R}^{1} \mathrm{COO}\right)_{2}$ can form stable complex with up to 2 free $\mathrm{R}^{1} \mathrm{COOH}$ 
molecules $\left(\mathrm{R}^{1}=\mathrm{H}\right)$ at $298 \mathrm{~K}$. The binding of $\mathrm{R}_{3}^{2} \mathrm{P}=\mathrm{Se}$ to $\mathrm{Cd}\left(\mathrm{R}^{1} \mathrm{COO}\right)_{2}\left(\mathrm{R}^{1} \mathrm{COOH}\right)_{\mathrm{x}}(\mathrm{x}=0,1$, 2) are favored for $x=0$ and 1 , but not for $x=2\left(R^{1}=R^{2}=H\right)$. Selected binding reactions and their corresponding free energy changes $\left(\Delta G^{298}\right)$ were tabulated in Table 4-4. Based on these free energy changes, the dominate species in a mixture of $\mathrm{R}_{3}^{2} \mathrm{P}=\mathrm{Se}$, $\mathrm{Cd}\left(\mathrm{R}^{1} \mathrm{COO}\right)_{2}$, and $\mathrm{R}^{1} \mathrm{COOH}$ at $298 \mathrm{~K}$ are $\mathrm{Cd}\left(\mathrm{R}^{1} \mathrm{COO}\right)_{2}\left(\mathrm{R}^{1} \mathrm{COOH}\right)_{2}$ and $\mathrm{R}_{3}^{2} \mathrm{P}=\mathrm{Se}-$ $\mathrm{Cd}\left(\mathrm{R}^{1} \mathrm{COO}\right)_{2}\left(\mathrm{R}^{1} \mathrm{COOH}\right)$. At high temperature, ligand binding to $\mathrm{Cd}\left(\mathrm{R}^{1} \mathrm{COO}\right)_{2}$ is less favored due to the large negative entropy change $\left(\Delta S^{298}\right)$ of these binding reactions. For example, dissociation of $\mathrm{R}_{3}^{2} \mathrm{P}=\mathrm{Se}-\mathrm{Cd}\left(\mathrm{R}^{1} \mathrm{COO}\right)_{2}\left(\mathrm{R}^{1} \mathrm{COOH}\right)$ into $\mathrm{R}_{3}^{2} \mathrm{P}=\mathrm{Se}-\mathrm{Cd}\left(\mathrm{R}^{1} \mathrm{COO}\right)_{2}$ and $\mathrm{R}^{1} \mathrm{COOH}$ is favored above $407 \mathrm{~K}$ and the same is true for $\mathrm{R}_{3}^{2} \mathrm{P}=\mathrm{Se}-\mathrm{Cd}\left(\mathrm{R}^{1} \mathrm{COO}\right)_{2}$ dissociation into $\mathrm{Cd}\left(\mathrm{R}^{1} \mathrm{COO}\right)_{2}$ and $\mathrm{R}_{3}^{2} \mathrm{P}=$ Se above $450 \mathrm{~K}$.

The formation of Se-Cd coordination bond between $\mathrm{PR}_{3}^{2} \mathrm{Se}$ and $\mathrm{Cd}\left(\mathrm{R}^{1} \mathrm{COO}\right)_{2}\left(\mathrm{R}^{1} \mathrm{COOH}\right)_{x}$ decreases the electron density on phosphorous atom and weakens the $\mathrm{P}=\mathrm{Se}$ bond. Both of the changes make the phosphine selenide more susceptible to nucleophilic attack. In the following sections, we will focus on the reaction of the $\mathrm{Cd}\left(\mathrm{R}^{1} \mathrm{COO}\right)_{2}\left(\mathrm{R}^{1} \mathrm{COOH}\right)_{\mathrm{x}}\left(\mathrm{R}_{3}^{2} \mathrm{P}=\mathrm{Se}\right)(\mathrm{x}=0)$ complexes.

\begin{tabular}{cccc}
\hline Reaction & $\Delta G^{298}$ & $\Delta H^{298}$ & $\Delta S^{298}$ \\
\hline $\mathrm{Cd}\left(\mathrm{R}^{1} \mathrm{COO}\right)_{2}+\mathrm{R}^{1} \mathrm{COOH} \rightarrow \mathrm{Cd}\left(\mathrm{R}^{1} \mathrm{COO}\right)_{2}\left(\mathrm{R}^{1} \mathrm{COOH}\right)$ & & & \\
\hline $\mathrm{Cd}\left(\mathrm{R}^{1} \mathrm{COO}\right)_{2}\left(\mathrm{R}^{1} \mathrm{COOH}\right)+\mathrm{R}^{1} \mathrm{COOH} \rightarrow \mathrm{Cd}\left(\mathrm{R}^{1} \mathrm{COO}\right)_{2}\left(\mathrm{R}^{1} \mathrm{COOH}\right)_{2}$ & -4.43 & -15.47 & -37.0 \\
$\mathrm{Cd}\left(\mathrm{R}^{1} \mathrm{COO}\right)_{2}+\mathrm{R}_{3}^{2} \mathrm{P}=\mathrm{Se} \rightarrow \mathrm{R}_{3}^{2} \mathrm{P}=\mathrm{Se}-\mathrm{Cd}\left(\mathrm{R}^{1} \mathrm{COO}\right)_{2}$ & -5.08 & -15.16 & -33.8 \\
$\mathrm{Cd}\left(\mathrm{R}^{1} \mathrm{COO}\right)_{2}\left(\mathrm{R}^{1} \mathrm{COOH}\right)+\mathrm{R}_{3}{ }_{3} \mathrm{P}=\mathrm{Se} \rightarrow \mathrm{R}_{3}{ }_{3} \mathrm{P}=\mathrm{Se}-\mathrm{Cd}\left(\mathrm{R}^{1} \mathrm{COO}\right)_{2}\left(\mathrm{R}^{1} \mathrm{COOH}\right)$ & -15.29 & -33.6 \\
$\mathrm{Cd}\left(\mathrm{R}^{1} \mathrm{COO}\right)_{2}\left(\mathrm{R}^{1} \mathrm{COOH}\right)_{2}+\mathrm{R}_{3}{ }_{3} \mathrm{P}=\mathrm{Se} \rightarrow \mathrm{R}_{3}{ }_{3} \mathrm{P}=\mathrm{Se}-\mathrm{Cd}\left(\mathrm{R}^{1} \mathrm{COO}\right)_{2}\left(\mathrm{R}^{1} \mathrm{COOH}\right)_{2}$ & -15.66 & -34.1 \\
$\mathrm{Cd}\left(\mathrm{R}^{1} \mathrm{COO}\right)_{2}\left(\mathrm{R}^{1} \mathrm{COOH}\right)_{2}+\mathrm{R}_{3}{ }_{3} \mathrm{P}=\mathrm{Se} \rightarrow \mathrm{R}_{3}{ }_{3} \mathrm{P}=\mathrm{Se}-\mathrm{Cd}\left(\mathrm{R}^{1} \mathrm{COO}\right)_{2}\left(\mathrm{R}^{1} \mathrm{COOH}\right)+\mathrm{R}^{1} \mathrm{COOH}$ & 0.22 & -38.9 \\
\hline
\end{tabular}


Table 4-4. Thermodynamics $\left(\Delta G^{298}\right.$ and $\left.\Delta H^{298}: \mathrm{kcal} \cdot \mathrm{mol}^{-1} ; \Delta S^{298}: \mathrm{cal}^{\prime} \cdot \mathrm{mol}^{-1} \cdot \mathrm{K}^{-1}\right)$ of ligand binding to $\mathrm{Cd}\left(\mathrm{R}^{1} \mathrm{COO}\right)_{2}\left(\mathrm{R}^{1}=\mathrm{R}^{2}=\mathrm{H}\right)$. These values have not been corrected for basis set superposition error (BSSE).

Reaction pathway of monomer formation. At relatively high temperature ( $>440 \mathrm{~K})$, $\mathrm{R}_{3}^{2} \mathrm{P}=\mathrm{Se}-\mathrm{Cd}\left(\mathrm{R}^{1} \mathrm{COO}\right)_{2} \quad \mathbf{1}\left(\mathrm{R}^{1}=\mathrm{R}^{2}=\mathrm{H}\right)$ is more stable than other $\mathrm{R}_{3}^{2} \mathrm{P}=\mathrm{Se}-$ $\mathrm{Cd}\left(\mathrm{R}^{1} \mathrm{COO}\right)_{2}\left(\mathrm{R}^{1} \mathrm{COOH}\right)_{\mathrm{x}}\left(\mathrm{x}=1,2 ; \mathrm{R}^{1}=\mathrm{R}^{2}=\mathrm{H}\right)$ complexes. In the following, we discuss the free energy changes for the decomposition of this complex at $573 \mathrm{~K}$, which is a typical temperature used in the synthesis of CdSe nanocrystals. The overall reaction pathway is shown in Scheme 4-2. The reported free energies were referenced to the isolated reagents.
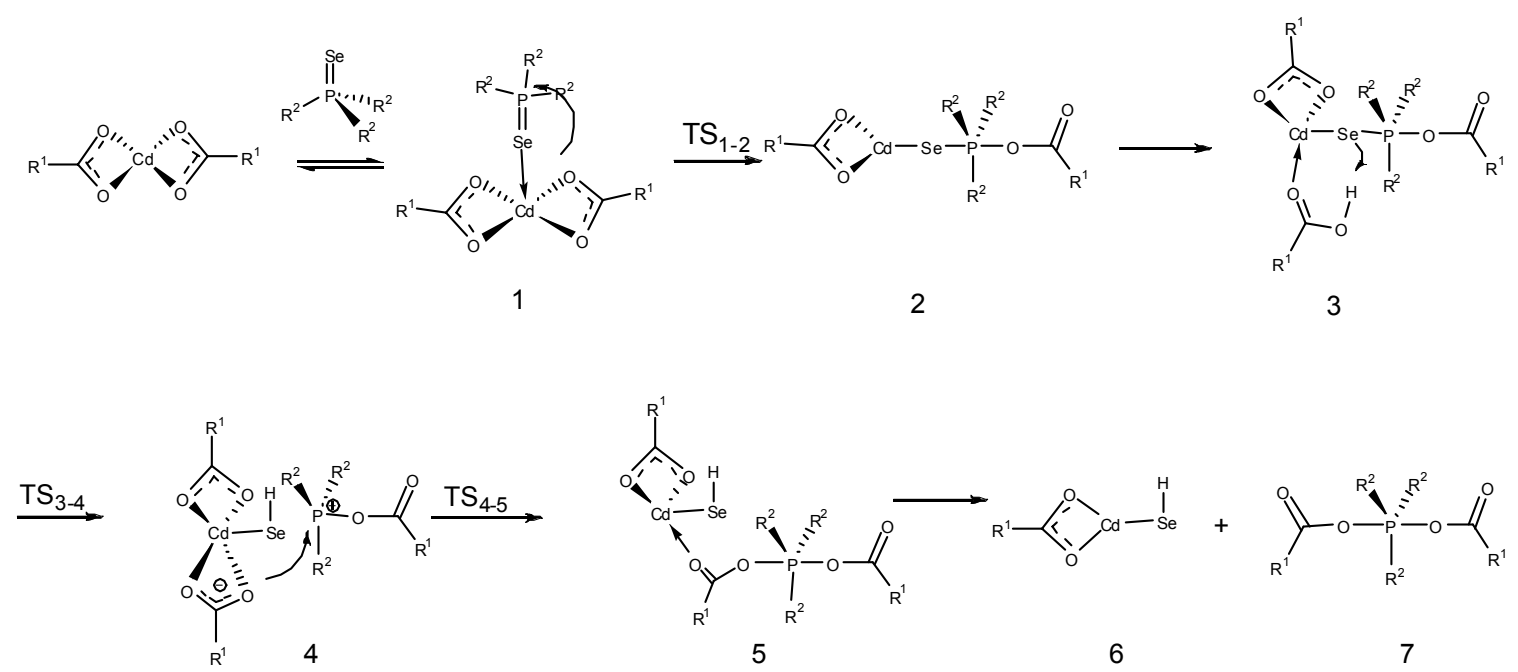

Scheme 4-2. Reaction Pathway of $\mathrm{R}_{3}^{2} \mathrm{P}=\mathrm{Se}-\mathrm{Cd}\left(\mathrm{R}^{1} \mathrm{COO}\right)_{2}$ complex

Formation of complex 1 from the two precursors is slightly uphill $\left(\Delta G^{573 \mathrm{~K}}=4.0\right.$ $\mathrm{kcal} \cdot \mathrm{mol}^{-1}$ ) at $573 \mathrm{~K}$, mainly due to the large entropic penalty of the molecular association 
process. $\mathrm{R}_{3}^{2} \mathrm{P}=\mathrm{Se}$ in complex $\mathbf{1}$ can be attacked by a carboxylate ligand of the same complex, overcoming a free energy barrier of $30.0 \mathrm{kcal} \cdot \mathrm{mol}^{-1}\left(\mathrm{TS}_{1-2}\right.$, Figure $\left.4-1\right)$ to give an intermediate $2\left(\Delta G^{573 \mathrm{~K}}=22.0 \mathrm{kcal} \cdot \mathrm{mol}^{-1}\right)$. This nucleophilic attack changes the phosphorous atom from a tetrahedral one in complex $\mathbf{1}$ into a distorted trianglebipyramidal one in the intermediate 2 . Formally, this reaction partially breaks the $\mathrm{P}=\mathrm{Se}$ bond to give a P-Se single bond and form a Cd-Se bond.

Other pathway to intermediate $\mathbf{2}$ was also explored. In particular, we find that intermolecular nucleophilic attack by free $\mathrm{R}^{1} \mathrm{COO}^{-}$ion is not likely. This is due to the low polarity of the solvent, which makes creation of charged species, such as $\mathrm{R}^{1} \mathrm{COO}^{-}$ion, energetically prohibitive. For example, $\Delta G^{573 \mathrm{~K}}$ of eq. (4-3) is calculated to be 48.3 $\mathrm{kcal} \cdot \mathrm{mol}^{-1}$ in $n$-heptane by using a polarization continuum solvation model (PCM). This free energy change is much higher than the activation free energies of the intra-molecular reaction pathways.

$\mathrm{R}_{3}^{2} \mathrm{P}=\mathrm{Se}-\mathrm{Cd}\left(\mathrm{R}^{1} \mathrm{COO}\right)_{2}\left(\mathrm{R}^{1} \mathrm{COOH}\right)_{2} \rightarrow\left[\mathrm{R}^{2}{ }_{3} \mathrm{P}=\mathrm{Se}-\mathrm{Cd}\left(\mathrm{R}^{1} \mathrm{COO}\right)\left(\mathrm{R}^{1} \mathrm{COOH}\right)_{2}\right]^{+}+\mathrm{HCOO}^{-}(4-3)$

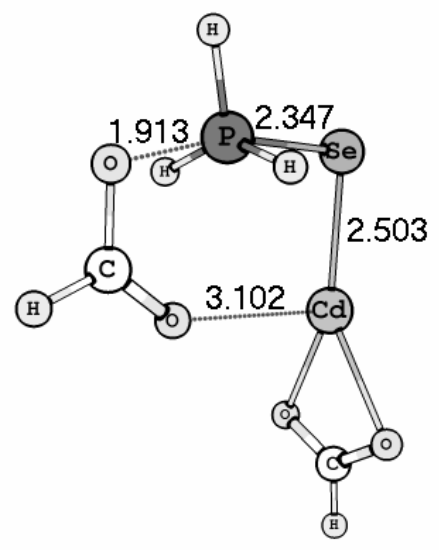

Figure 4-1. Structure of $\mathrm{TS}_{1-2}$. The unit of the bond length is $\AA$. 
Further weakening of the bonding between Se and $\mathrm{P}$ is realized via proton transfer from a $\mathrm{R}^{1} \mathrm{COOH}$ surfactant molecule to the Se atom of 2 . During this process, a $\mathrm{R}^{1} \mathrm{COOH}$ molecule binds to the $\mathrm{Cd}$ and $\mathrm{Se}$ atoms of $\mathbf{2}$ using its carbonyl oxygen and acidic proton, respectively, to give $\mathbf{3}\left(\Delta G^{573 \mathrm{~K}}=31.8 \mathrm{kcal} \cdot \mathrm{mol}^{-1}\right)$. The Se atom in $\mathbf{3}$ accepts the acidic proton from $\mathrm{R}^{1} \mathrm{COOH}$ forming an ion pair $4\left(\Delta G^{573 \mathrm{~K}}=34.6 \mathrm{kcal} \cdot \mathrm{mol}^{-1}\right)$. This reaction formally breaks the covalent bond between $\mathrm{P}$ and Se atoms. The activation free energy $\left(\mathrm{TS}_{3-4}\right.$, Figure 4-2) of this proton transfer reaction is $42.7 \mathrm{kcal} \cdot \mathrm{mol}^{-1}$, the highest of all the transition states.

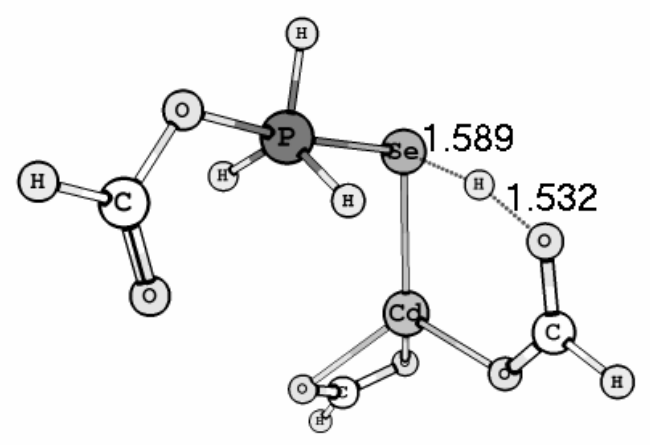

Figure 4-2. Structure of $\mathrm{TS}_{3-4}$

The phosphorous atom of the ion pair can be attacked by another carboxylate ligand of the same complex via transition state $\mathrm{TS}_{4-5}$ to give $5\left(31.0 \mathrm{kcal} \cdot \mathrm{mol}^{-1}\right)$. Complex 5 has a $\mathrm{R}^{1} \mathrm{COO}-\mathrm{PR}^{2}{ }_{3}$-OCOR ${ }^{1}$ moiety that coordinates to $\mathrm{Cd}^{2+}$ using one of its carbonyl oxygen atoms. Dissociation of this $\mathrm{R}^{1} \mathrm{COO}-\mathrm{PR}^{2}{ }_{3}-\mathrm{OCOR}^{1}$ molecule from the cadmium atom gives a $\mathrm{Cd}\left(\mathrm{R}^{1} \mathrm{COO}\right)(\mathrm{Se}-\mathrm{H})$ complex 6 and a $\mathrm{R}^{1} \mathrm{COO}-\mathrm{PR}^{2}{ }_{3}-\mathrm{OCOR}^{1}$ molecule 7 (20.0 $\left.\mathrm{kcal} \cdot \mathrm{mol}^{-1}\right)$.

The $\mathrm{Cd}(\mathrm{Se}-\mathrm{H})\left(\mathrm{R}^{1} \mathrm{COO}\right)$ complex 6 can be viewed as the basic growth unit of CdSe clusters and nanocrystals. A detailed study of the nanocrystal growth process from 
the $\mathrm{Cd}(\mathrm{Se}-\mathrm{H})\left(\mathrm{R}{ }^{1} \mathrm{COO}\right)$ complex is underway. $\mathrm{R}^{1} \mathrm{COO}-\mathrm{PR}^{2}{ }_{3}-\mathrm{OCOR}^{1} 7$ can exist in either cis or trans conformation, depending on the relative orientation of the two carbonyl groups. The barrier of the cis-trans transition is very low. The cis conformer can undergo a unimolecular decomposition reaction, in which the equatorial carbonyl oxygen attacks the axial carbonyl carbon, breaking the axial $\mathrm{C}-\mathrm{O}$ bond and the equatorial $\mathrm{P}-\mathrm{O}$ bond to produce phosphine oxide and carboxylic acid anhydride (Scheme 4-2). These two decomposition products are the only other major reaction products observed experimentally besides CdSe nanocrystals. The activation free energy of this reaction is relatively low (Figure $4-4, \Delta G^{\$ 573}=22.3 \mathrm{kcal} \cdot \mathrm{mol}^{-1}$, referenced to the trans conformer) while the total free energy of $\mathrm{Cd}(\mathrm{Se}-\mathrm{H})\left(\mathrm{R}^{1} \mathrm{COO}\right)$ complex, phosphine oxide, and carboxylic acid anhydride is $1.0 \mathrm{kcal} \cdot \mathrm{mol}^{-1}$ relative to the precursors.

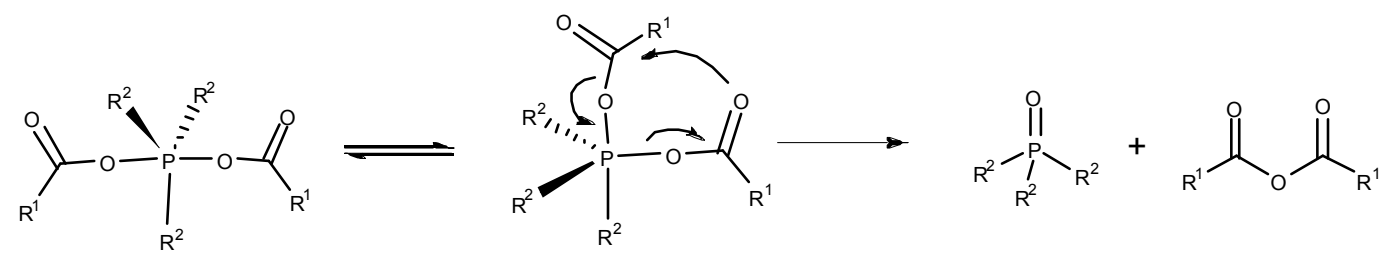

Scheme 4-2. Unimolecular decomposition of $\mathrm{R}^{1} \mathrm{COO}-\mathrm{PR}^{2}{ }_{3}-\mathrm{OCOR}{ }^{1}$

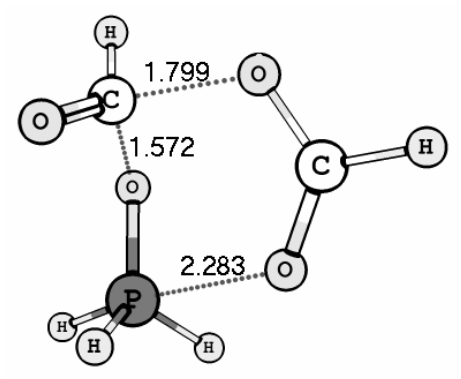

Figure 4-4 Transition state of the unimolecular decomposition of $\mathrm{R}^{1} \mathrm{COO}-\mathrm{PR}^{2}{ }_{3}-\mathrm{OCOR}^{1}$ $\left(\mathrm{R}^{1}=\mathrm{R}^{2}=\mathrm{H}\right)$. 


\subsection{Conclusion}

In summary, I have calculated the free energy landscape of the precursor decomposition and monomer formation of the synthesis of CdSe nanocrystals within B3LYP level of theory. The calculation shows that phosphine selenide is first activated by binding to cadmium carboxylate, which is followed by an intramolecular nucleophilic attack by the carboxylate. Carboxylic acid surfactant plays an important role in the cleavage of $\mathrm{P}=\mathrm{Se}$ bond by supplying proton to the selenium atom. Our result reveals the general features of the precursor to monomer conversion pathway and offers a theoretical ground for studying the nanocrystal nucleation and growth kinetics. 


\section{References}

(1) Lin, S. L.; Pradhan, N.; Wang, Y. J.; Peng, X. G. Nano Lett. 2004, 4, 2261-2264.

(2) A. A. Granovsky, PC GAMESS version 7.0, http://classic.chem.msu.su/gran/gamess/index.html. Accessed Jan 2007.

(3) Becke, A. D. J. Chem. Phys. 1993, 98, 5648-5652.

(4) Lee, C. T.; Yang, W. T.; Parr, R. G. Phys. Rev. B 1988, 37, 785-789.

(5) Vosko, S. H.; Wilk, L.; Nusair, M. Can. J. Phys. 1980, 58, 1200-1211.

(6) Stephens, P. J.; Devlin, F. J.; Chabalowski, C. F.; Frisch, M. J. J. Phys. Chem. 1994, 98, 11623-11627.

(7) Basis sets were obtained from the Extensible Computational Chemistry Environment Basis Set Database, Version 02/02/06, as developed and distributed by the Molecular Science Computing Facility, Environmental and Molecular Sciences Laboratory which is part of the Pacific Northwest Laboratory, P.O. Box 999, Richland, Washington 99352, USA, and funded by the U.S. Department of Energy. The Pacific Northwest Laboratory is a multi-program laboratory operated by Battelle Memorial Institute for the U.S. Department of Energy under contract DE-AC06-76RLO 1830. Contact Karen Schuchardt for further information.

(8) Martin, J. M. L.; Sundermann, A. J. Chem. Phys. 2001, 114, 3408-3420.

(9) Woon, D. E.; Dunning, T. H. J. Chem. Phys. 1993, 98, 1358-1371.

(10) Hehre, W. J.; Ditchfie.R; Pople, J. A. J. Chem. Phys. 1972, 56, 2257-\&.

(11) Hay, P. J.; Wadt, W. R. J. Chem. Phys. 1985, 82, 270-283.

(12) Hay, P. J.; Wadt, W. R. J. Chem. Phys. 1985, 82, 299-310. 
(13) Wadt, W. R.; Hay, P. J. J. Chem. Phys. 1985, 82, 284-298.

(14) Check, C. E.; Faust, T. O.; Bailey, J. M.; Wright, B. J.; Gilbert, T. M.; Sunderlin, L. S. J. Phys. Chem. A 2001, 105, 8111-8116.

(15) Peterson, K. A.; Figgen, D.; Goll, E.; Stoll, H.; Dolg, M. J. Chem. Phys. 2003, 119, 11113-11123.

(16) Gilheany, D. G. In The chemistry of organophosphorus compounds; Hartley, F. R., Ed.; Jon Wiey \& Sons: 1992; Vol. 2.

(17) Kramolowsky, R.; Sawluk, J.; Siasios, G.; Tiekink, E. R. T. Inorg Chim Acta 1998, 269, 317-321.

(18) Liu, H.; Owen, J. S.; Alivisatos, A. P. J. Am. Chem. Soc. 2007, 129, 305312.

(19) Liu, H.T.; Alivisatos, A. P., Unpublished 


\section{Chapter 5}

\section{Effect of Water on the Synthesis of}

\section{CdSe and CdS Nanorods}

\subsection{Introduction}

The growth anisotropy of CdSe, CdS, and other group II-VI materials has made it possible to synthesize non-spherical shaped nanocrystals of this class. ${ }^{1-4}$ Both experimental $^{2,3}$ and theoretical ${ }^{5-7}$ studies have suggested that adsorption of surfactant molecules on the nanocrystal surface is one of the major mechanisms of the anisotropic growth. Based on these results, it has been suggested that by tuning the binding strength of surfactant to the nanocrystal surface, it might be possible to precisely engineering the growth of nanocrystals. So far, a number of organic ligands, including phosphonic acid, carboxylic acid, and amine, have been used in the synthesis of group II-VI nanocrystals. The binding energy of these ligands is determined by the nature of their head groups. To the best of our knowledge, no clear correlation between the binding strength and nanocrystal shape has been demonstrated.

In the previous chapters, I have shown that during the synthesis of CdSe nanocrystals using phosphonic acid as surfactant, ${ }^{8}$ poly-phosphonic acid anhydride was one of the major reaction products. The fate of phosphonic acid anhydride depends on the 
moisture content of the reaction mixture. Under anhydrous conditions, significant amount of phosphonic acid anhydride accumulates in the reaction mixture. However, in cases where the reaction mixture is not strictly anhydrous, the anhydride product undergoes hydrolysis reaction to produce monomeric form of phosphonic acid. The first case is represented by the synthesis of CdSe using $\mathrm{CdMe}_{2}$ as the cadmium precursor, while the second case is true for the $\mathrm{CdSe}$ synthesis using $\mathrm{CdO}$ as the precursor. In the $\mathrm{CdO}$ based syntheses, one equivalent of water was produced during the dissolution of $\mathrm{CdO}$ according to equation (5-1) ( $\mathrm{H}_{2}$-PA: $n$-alkylphosphonic acid, Cd-PA: cadmium $n$ alkylphosphonate). ${ }^{8,9}$ The water generated by equation (5-1) condenses on the sidewall of the reaction vessel and serves as a reservoir of water vapor that diffuses to the reaction mixture during the nanocrystal growth. ${ }^{8}$

$$
\mathrm{CdO}+\mathrm{H}_{2}-\mathrm{PA} \rightarrow \mathrm{Cd}-\mathrm{PA}+\mathrm{H}_{2} \mathrm{O}
$$

Poly-phosphonic acid anhydride is a polydentate ligand that should bind to surface cadmium atoms of CdSe nanocrystal much stronger than monomeric form of phosphonic acid does. Since the presence of water controls the concentration of polyphosphonic acid anhydride in the reaction mixture; we can therefore predict that water should significantly affect the degree of surface passivation of CdSe nanocrystals during its growth. Such change in the surface coverage should significantly change the size and shape evolution of CdSe nanocrystals. Specifically, CdSe nanorods grown in the presence of water should be fatter than the ones grown under anhydrous conditions, since it has been shown that surfactant mostly bind to the side facets of the nanorods.

Interestingly, it has been known that the CdSe nanorod synthesized using $\mathrm{CdMe}_{2}$ has very different morphology from that prepared using CdO. The CdSe nanorod 
prepared using $\mathrm{CdMe}_{2}$ is usually thinner and has larger aspect ratio than that prepared using $\mathrm{CdO}$. In light of the differences in the chemistry between the two types of syntheses, we suspect that the water is responsible for the differences in the nanorod morphology.

\subsection{Experimental}

Tri- $n$-octylphosphine (TOP, 97\%, Strem), tri- $n$-butylphosphine (TBP, 99\%, Strem), TOPO (Aldrich or Arcos, 99\%. The same batch of TOPO was used whenever two experiments were to be compared), n-octadecylphosphonic acid $\left(\mathrm{H}_{2}\right.$-ODPA, Polycarbon), n-hexylphosponic acid ( $\mathrm{H}_{2}$-HPA, Polycarbon), CdO (Aldrich, 99.99+\%), $\mathrm{Ac}_{2} \mathrm{O}$ (99\%, EMD chemicals), Selenium (99.99\%, Aldrich), and Sulfur (99.99\%, Aldrich) were used as received. Standard air sensitive techniques were used to handle air and moisture sensitive compounds.

Synthesis of $\mathrm{CdE}(\mathrm{E}=\mathrm{S}$, Se) nanocrystal with vacuum drying. CdSe: To a 25

$\mathrm{mL}$ three-neck flask equipped with a condenser and a thermocouple adapter was added TOPO (2.73 g, $7.06 \mathrm{mmol}), \mathrm{H}_{2}$-ODPA (1.07 g, $\left.3.20 \mathrm{mmol}\right)$, and CdO (0.204 g, 1.60 mmol). The mixture was degassed at $120^{\circ} \mathrm{C}$ and $200-400$ mtorr pressure for $60 \mathrm{~min}$. The flask was then filled with $\mathrm{Ar}$ and the temperature was raised to $320^{\circ} \mathrm{C}$ to dissolve $\mathrm{CdO}$. After dissolving $\mathrm{CdO}$, the temperature was lowered to $150{ }^{\circ} \mathrm{C}$ and the pressure was reduced to $\sim 300$ mtorr for $60 \mathrm{~min}$. The flask was then filled with Ar and the temperature was raised to $270{ }^{\circ} \mathrm{C}$. Pure TOPSe $(0.70 \mathrm{~g}, 1.6 \mathrm{mmol})$ was injected and the temperature was allowed to stabilize at $260 \pm 2{ }^{\circ} \mathrm{C}$. The amount of TOPSe injected $(1.4 \pm 0.1 \mathrm{mmol})$ was measured as the difference between the mass of the syringe before and after the 
injection. Aliquots taken after the injection of TOPSe were dissolved in toluene and used for TEM and UV-Vis measurements without any purification. CdS: CdS nanorod was prepared similarly using a mixture of TOPS $(1.6 \mathrm{mmol})$ and toluene $(0.30 \mathrm{~g})$ as the injection solution. The injection and growth temperatures were $320^{\circ} \mathrm{C}$ and $290{ }^{\circ} \mathrm{C}$, respectively.

Synthesis of CdE nanocrystal with added water. After the reaction mixture was dried under reduced pressure after the dissolution of $\mathrm{CdO}$ as described in the previous paragraph, the temperature was further lowered to $90{ }^{\circ} \mathrm{C}$ and water $(100 \mathrm{mg})$ was added to the flask via a syringe under Ar. The reaction was then heated to the injection temperature and TOPE was injected as described above.

Synthesis of CdSe nanocrystal with $\mathbf{A c}_{2} \mathbf{O}$ drying. To a $25 \mathrm{~mL}$ three-neck flask equipped with a condenser and a thermocouple adapter was added TOPO (3.00 g, 7.77 mmol), $\mathrm{H}_{2}$-ODPA (0.85 g, $\left.2.54 \mathrm{mmol}\right), \mathrm{H}_{2}$-HPA (0.16 g, $\left.0.96 \mathrm{mmol}\right)$ and CdO (0.199 g, $1.60 \mathrm{mmol}$ ). The mixture was degassed at $120{ }^{\circ} \mathrm{C}$ and $200-400$ mtorr pressure for 15 min. The flask was then filled with $\mathrm{Ar}$ and heated to $320{ }^{\circ} \mathrm{C}$ to dissolve $\mathrm{CdO}$. After dissolving $\mathrm{CdO}$, the temperature was lowered to $120^{\circ} \mathrm{C}$ and the pressure was reduced to $\sim 200$ mtorr for $20 \mathrm{~min}$. The flask was then filled with $\mathrm{Ar}$ before $\mathrm{Ac}_{2} \mathrm{O}(0.50 \mathrm{~mL})$ was added via a syringe and allowed to react for $2-3$ hours at $120{ }^{\circ} \mathrm{C}$. Excess $\mathrm{Ac}_{2} \mathrm{O}$ was removed with an Ar stream after which the reaction mixture was degassed at $150{ }^{\circ} \mathrm{C}, 200$ mtorr for $10 \mathrm{~min}$. The flask was then filled with $\mathrm{Ar}$ and heated to $270{ }^{\circ} \mathrm{C}$. TBPSe $(0.15$ $\mathrm{mL}$ ) was injected to the reaction mixture via a syringe. The temperature was set to $260{ }^{\circ} \mathrm{C}$ after the injection and the total reaction time is $30 \mathrm{~min}$. After the first TBPSe injection, TBPSe was added to the reaction mixture via a 21 gauge disposable needle at a rate of 1 
drop every $20 \mathrm{~s}$ in the first 20 min of reaction and 1 drop every $15 \mathrm{~s}$ after 20 min of reaction. The total amount of TBPSe added was $0.59 \mathrm{~g}(2.1 \mathrm{mmol})$. For the CdSe nanocrystal synthesis carried out without drying, the reaction mixture was cooled to 120 ${ }^{\circ} \mathrm{C}$ without degassing and then heated up $270{ }^{\circ} \mathrm{C}$ before TBPSe was injected. For the control experiment with added water, water $(29 \mathrm{mg}, 1.6 \mathrm{mmol})$ was added to the reaction mixture via a syringe at $120{ }^{\circ} \mathrm{C}$ after excess $\mathrm{Ac}_{2} \mathrm{O}$ was removed by $\mathrm{Ar}$ stream and degassing and before TBPSe injection.

Time required to develop a red color in the reaction mixture were 4 min (with $\mathrm{Ac}_{2} \mathrm{O}$ drying), $30 \mathrm{~s}$ (no drying), and $20 \mathrm{~s}$ (with $\mathrm{Ac}_{2} \mathrm{O}$ drying then added water).

Computational methods. All calculations were carried out using pcGAMESS $\operatorname{program}^{10}$ at the $\mathrm{B}^{2} \mathrm{LYP}^{11-14}$ (using VWN3 functional) level of theory. The following basis sets ${ }^{15}$ were used: Lanl2dzdp basis without the diffusion function and the associated

effective core potential (ECP) for $\mathrm{Cd}$, Se, and $\mathrm{P}^{16}$ and $6-31 \mathrm{G}^{*}$ basis for $\mathrm{O}, \mathrm{C}$, and $\mathrm{H}^{17}$ The molecular geometries were relaxed in vacuum without constrain until the maximum energy gradient is smaller than $5 \times 10^{-5}$ hartree/bohr. Frequency calculation was performed at the same level of theory to confirm that the obtained geometry is a local minimum. Thermodynamic properties were calculated at $300 \mathrm{~K}$ assuming rigid rotor model and ideal gas behavior and referenced to the free surfactant and $(\mathrm{CdSe})_{6}$ cluster.

\subsection{Results and Discussion}

Density functional theory calculations were first carried out to compare the binding energy of phosphonic acid anhydride and regular phosphonic acid to CdSe nanocrystal surface. Since only relative binding energy was needed to compare the two 
surfactants, we used a $(\mathrm{CdSe})_{6}$ cluster to model CdSe nanocrystal (Figure 5-1). As expected, the di-methyl-phosphonic acid anhydride binds to $(\mathrm{CdSe})_{6}$ much stronger $\left(\Delta G^{298}=21.6 \mathrm{kcal} \cdot \mathrm{mol}^{-1}\right)$ than methyl-phosphonic acid does $\left(\Delta G^{298}=14.6 \mathrm{kcal} \cdot \mathrm{mol}^{-1}\right) . \mathrm{In}$ view of this result, we expect that the growth of CdSe nanocrystal should be slower in the presence of poly-phosphonic acid anhydride.
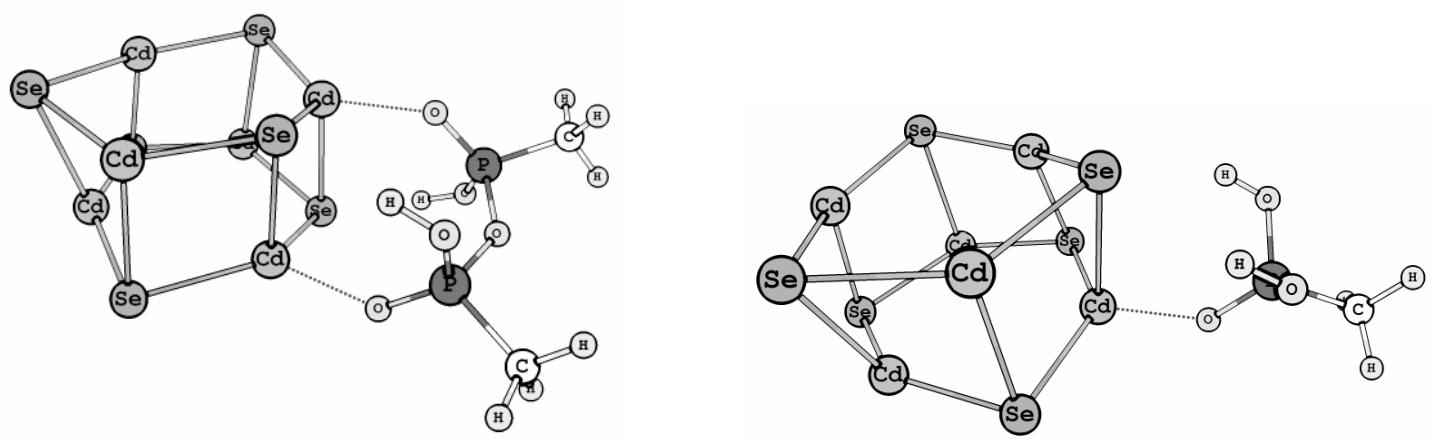

Figure 5-1. Optimized structure of (left) di-methyl-phosphonic acid anhydride and (right) methyl-phosphonic acid complex with $\mathrm{Cd}_{6} \mathrm{Se}_{6}$.

To verify this theoretical prediction, we have synthesized $\mathrm{CdE}(\mathrm{E}=\mathrm{S}, \mathrm{Se})$ nanorods by an injection of TOPE (tri- $n$-octylphosphine selenide or sulfide) to a TOPO solution of Cd-PA at high temperature. To carry out the nanocrystal synthesis in an anhydrous environment, the reaction mixture was dried under reduced pressure (200 mtorr, $150{ }^{\circ} \mathrm{C}$, for 1 hour) between the dissolution of $\mathrm{CdO}$ and the injection of TOPE. This procedure has been shown to remove at least $90 \%$ of the water generated by equation (5-1). ${ }^{8}$ To demonstrate the effect of water on the growth of CdE nanorod, control experiments were carried out where water was added to the reaction between the vacuum drying and the injection of TOPE. Care was taken to ensure that other reaction conditions stayed the same for the two syntheses. 
After the injection of TOPE, the reaction mixture gradually developed a red or yellow color, indicating the nucleation and growth of CdSe and CdS nanocrystals, respectively. It was observed that this color change was faster in the reactions carried out with added water than their dried counterparts, which is consistent with faster reaction rate with added water. ${ }^{8}$ An increase in the diameter and decrease in the average aspect ratio of the nanorod was observed when the reaction was carried out with added water. For example, the average aspect ratio of the CdS nanorod prepared with vacuum drying is 20 (nanorod dimension: $92 \pm 16 \mathrm{~nm} \times 4.5 \pm 0.5 \mathrm{~nm}$, the errors represent one standard deviation of the measurements) at $40 \mathrm{~min}$ after the TOPS injection, while that of the CdS nanorod prepared with added water is only $8(57 \pm 13 \mathrm{~nm} \times 7.0 \pm 0.8 \mathrm{~nm})$ at the same reaction time (Figure 5-2, A B). A similar observation was made in the two CdSe nanorod samples taken at 9 min (Figure 5-2, C D). The average aspect ratio of the CdSe nanorod decreased from 2.8 (with vacuum drying, $14.5 \pm 2.0 \mathrm{~nm} \times 5.2 \pm 0.6 \mathrm{~nm}$ ) to 1.7 (with added water, $9.9 \pm 1.1 \mathrm{~nm} \times 5.8 \pm 0.6 \mathrm{~nm}$ ) upon addition of water. Although the reaction conditions have not been optimized to prepare mono-dispersed materials, the dependence of the nanorod diameter and aspect ratio on the presence of water is significant in both cases. 

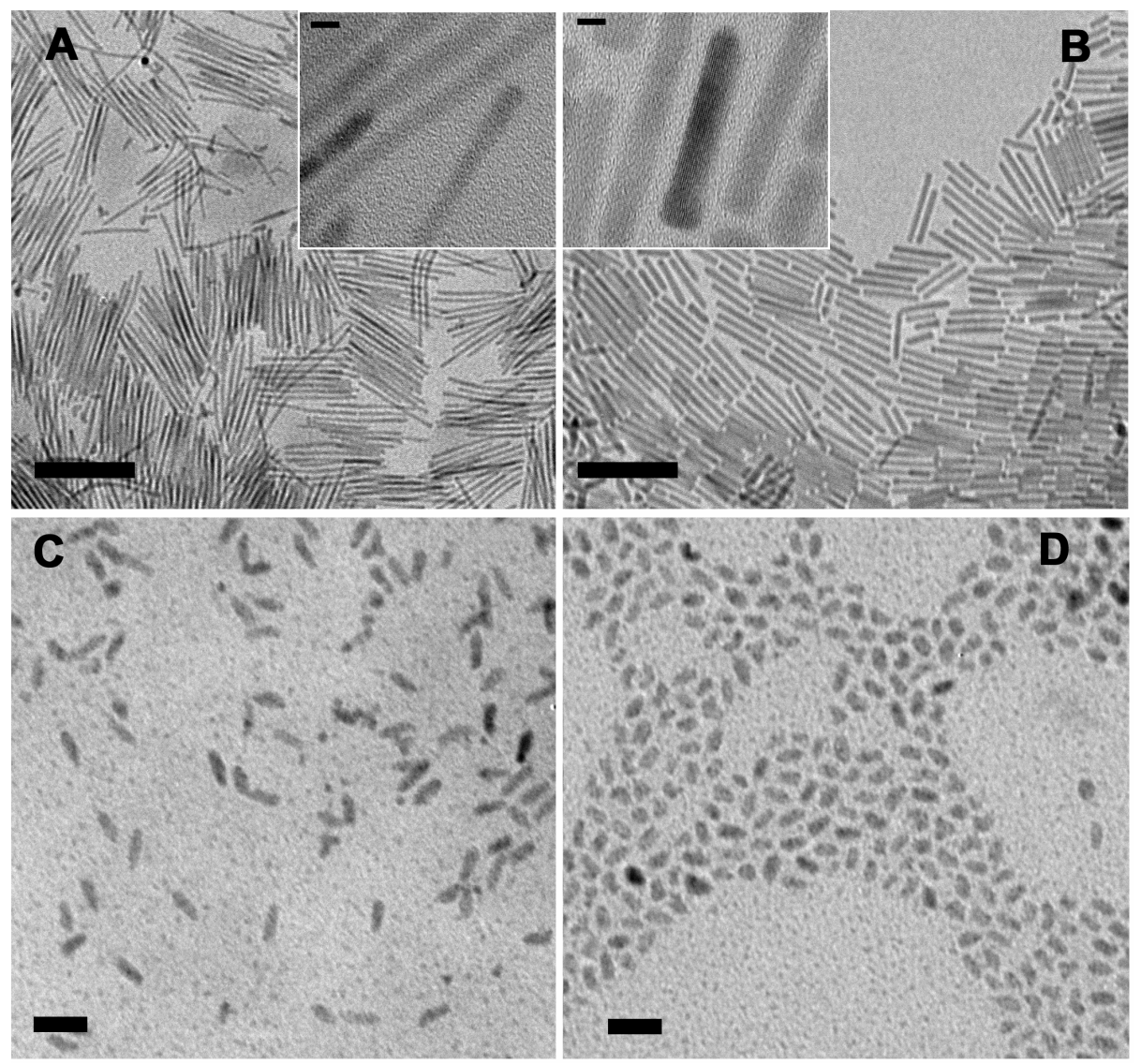

Figure 5-2. Transmission electron micrographs of CdS nanocrystals prepared (A) with vacuum drying and (B) with added water, and CdSe nanocrystals prepared (C) with vacuum drying (D) with added water. The insets are high resolution images of CdS nanocrystal. Scale bars represent $100 \mathrm{~nm}$ for CdS, $5 \mathrm{~nm}$ for the insets, and $20 \mathrm{~nm}$ for CdSe, respectively.

UV-Vis absorption spectroscopy was used to follow the time evolution of the CdSe nanorod diameter, since most of the quantum confinement effect of the nanorod originates from this dimension. ${ }^{18}$ At the same reaction time, the $1^{\text {st }}$ exciton peak of the CdSe sample prepared with added water is always red-shifted compared to that grown under anhydrous conditions (Figure 5-3, bottom left). This red shift shows that the 
presence of water increases the growth rate of the diameter, consistent with the TEM measurements. In addition, an absorption peak at $350 \mathrm{~nm}$ appeared in the UV-Vis spectrum at early reaction times when the reaction was dried with reduced pressure (Figure 5-3, C). Such an absorption feature was not observed in the synthesis with added water (Figure 5-3, D). Similarly, an absorption peak at $310 \mathrm{~nm}$ appeared in the CdS synthesis only when the reaction was dried with reduced pressure. The $350 \mathrm{~nm}$ peak that appeared in the CdSe synthesis has been suggested to arise from a CdSe cluster and its appearance was shown to be correlated with the anisotropic growth of CdSe nanocrystal. ${ }^{4}$ Indeed, the two vacuum-dried reactions produced nanocrystals with smaller diameter and higher aspect ratio. Thus it appears that water reduces the growth anisotropy of CdS and CdSe nanocrystals by increasing the growth rate in the diameter dimension.

In the above experiments, water was not quantitatively removed. In addition, poly-phosphonic acid was formed by the reaction between the precursors and its amount was limited by the conversion of the precursor. To circumvent these limitations, a more effective method was used to probe the role of poly-phosphonic acid in the synthesis of CdSe nanorods. Briefly, acetic acid anhydride $\left(\mathrm{Ac}_{2} \mathrm{O}\right)$ was added to the reaction after the dissolution of $\mathrm{CdO}$ and allowed to react at $120{ }^{\circ} \mathrm{C}$ for $2-3$ hours. Water was removed according to equation (5-2) (HOAc: acetic acid). In addition, phosphonic acid could also be dehydrated to produce poly-phosphonic acid (5-3) in the process

$$
\begin{aligned}
& \mathrm{Ac}_{2} \mathrm{O}+\mathrm{H}_{2} \mathrm{O} \rightarrow 2 \mathrm{HOAc} \\
& \mathrm{H}_{2}-\mathrm{PA}+\mathrm{Ac}_{2} \mathrm{O} \rightarrow \text { poly- } \mathrm{H}_{2}-\mathrm{PA}+2 \mathrm{HOAc}
\end{aligned}
$$


Excess $\mathrm{Ac}_{2} \mathrm{O}$ and any $\mathrm{HOAc}$ formed by (5-2) and (5-3) were removed under reduced pressure at $150^{\circ} \mathrm{C}$ (b.p. of $\mathrm{Ac}_{2} \mathrm{O}: 139^{\circ} \mathrm{C}$, HOAc: $118^{\circ} \mathrm{C}$ ). Multiple injections of selenium precursor were used in this set of experiments.
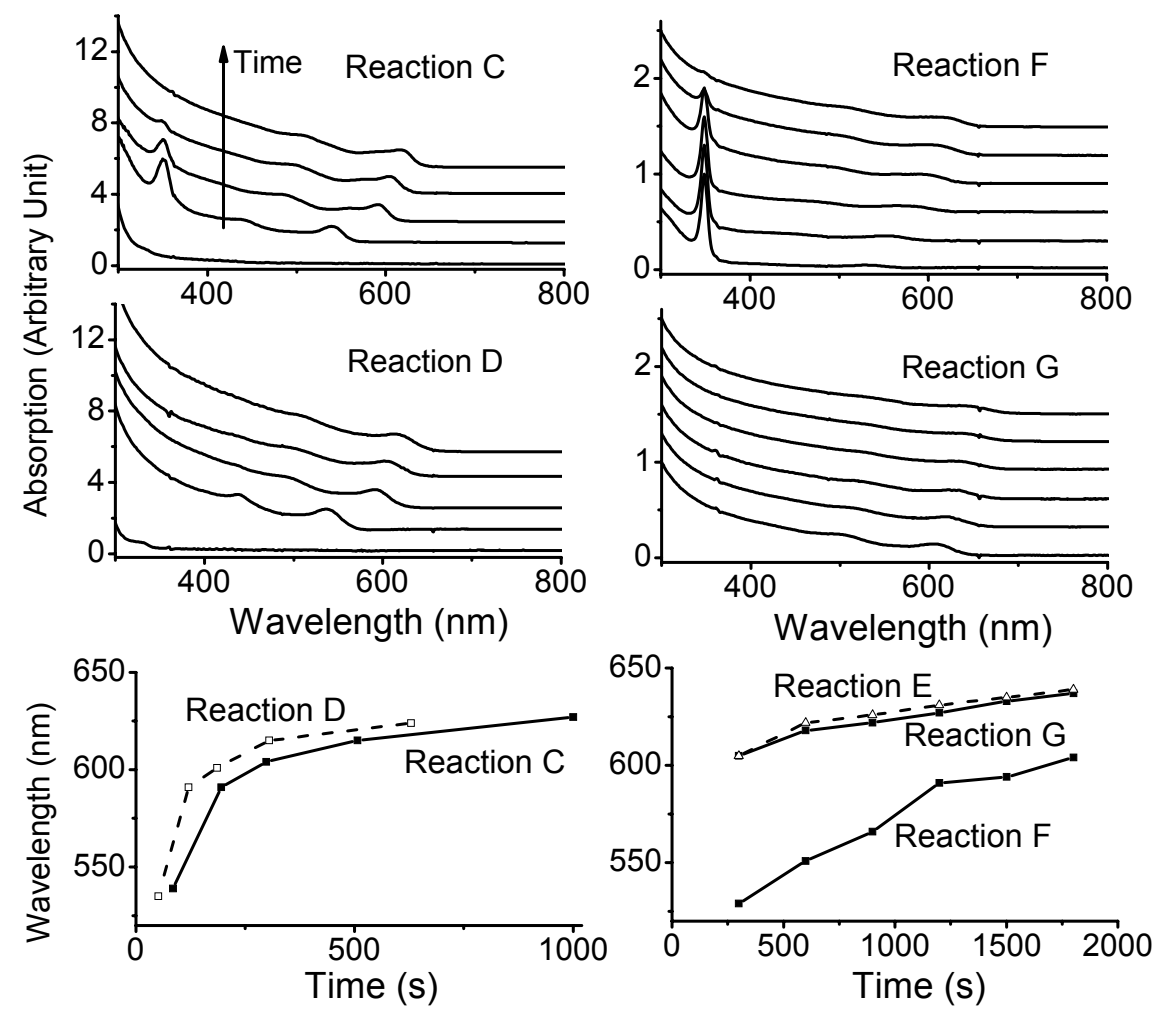

Figure 5-3. UV-Vis spectra of the aliquots taken in reaction (C) at 13, 85, 195, 298, 507 seconds, and reaction (D) at 6, 51, 120, 185, 305 seconds; reaction (F) and (G) at 5, 10, 15, 20, 25, $30 \mathrm{~min}$ after Se precursor injection. The bottom curves show time evolution of the first exciton peak position in the reactions.

In the reaction carried out without any drying (Figure 5-4, E), the growth of CdSe nanorod in the long axis essentially stopped after the first five minutes, even though Se 
precursor was continuously added throughout the reaction. The reaction with $\mathrm{Ac}_{2} \mathrm{O}$ drying (Figure 5-4, F) showed distinctly different growth kinetics and nanocrystal morphology from reaction E. Especially, the growth in the length continued throughout the reaction and the maximum length of the nanorod increased to more than 3 times that of sample E. To show that the changes in the growth kinetics and nanocrystal morphology are due to the rigorous removal of water by $\mathrm{Ac}_{2} \mathrm{O}$ treatment, a control experiment was carried out in which the reaction mixture was first dried with $\mathrm{Ac}_{2} \mathrm{O}$ and then added $29 \mathrm{mg}$ (amount of water generated from CdO: $30 \mathrm{mg}$ ) of water. This control experiment produced essentially the same growth kinetics and nanocrystal morphology as those observed in reaction E (Figure 5-3 and 5-4, G).

UV-Vis spectra of the aliquots taken from the above three CdSe syntheses showed similar features to those observed in the vacuum-dried reactions. First, the $1^{\text {st }}$ exciton peaks of sample $\mathrm{E}$ and $\mathrm{G}$ are substantially red shifted compared to that of sample F, which suggests that the presence of water increased the growth rate of the nanorod diameter (Figure 5-3, bottom right). In addition, a sharp peak at $350 \mathrm{~nm}$ was observed in sample $\mathrm{F}$ for up to $25 \mathrm{~min}$ after the first injection of TBPSe (Figure 5-3, F). In contrast, such an adsorption feature was not observed in sample $\mathrm{E}$ and $\mathrm{G}$ even in the first sample taken at $5 \mathrm{~min}$. Again, the extended appearance of the $350 \mathrm{~nm}$ peak in sample $\mathrm{F}$ is linked with the rigorous removal of water and the enhanced anisotropic growth of the nanorod. These observations further support the hypothesis that the presence of water decreases the growth anisotropy of $\mathrm{CdSe}$ and $\mathrm{CdS}$ nanocrystals by increasing the growth rate in the diameter dimension under our reaction conditions. 

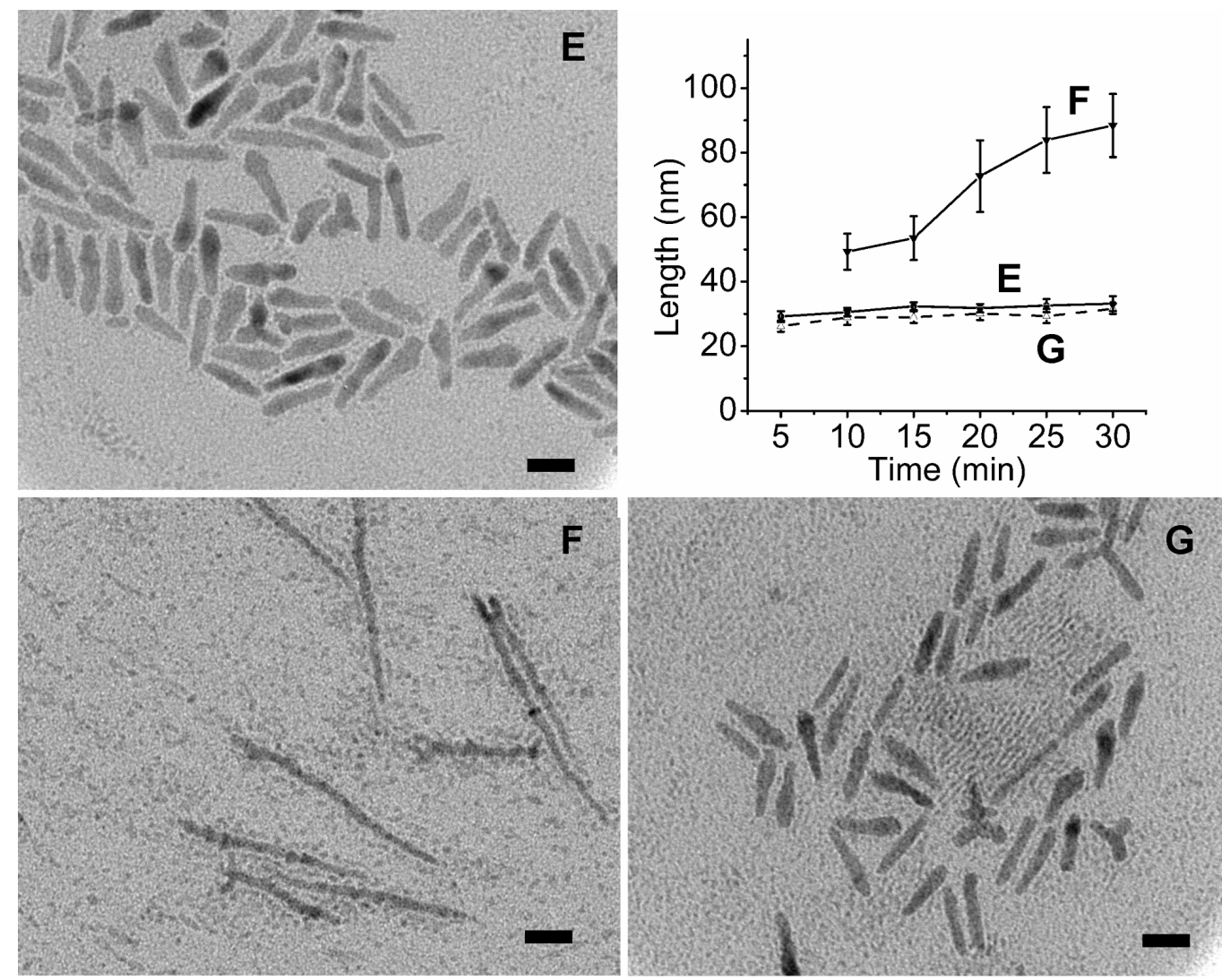

Figure 5-4. TEM images of CdSe nanorods prepared by (E) without any drying (F) dried with $\mathrm{Ac}_{2} \mathrm{O}$ treatment $(\mathrm{G})$ dried with $\mathrm{Ac}_{2} \mathrm{O}$ then added $20 \mathrm{mg}$ of $\mathrm{H}_{2} \mathrm{O}$. The top right graph shows the time evolution of the nanorod length. The TEM samples were taken at $30 \mathrm{~min}$ after the reaction. The scale bars represent $20 \mathrm{~nm}$. The error bars represent one standard deviation.

\subsection{Conclusions}

In summary, we have shown that poly-phosphonic acid anhydride is an effective surfactant to produce high-aspect ratio $\mathrm{CdSe}$ and $\mathrm{CdS}$ nanorod. The presence of water decreases the growth anisotropy of $\mathrm{CdSe}$ and $\mathrm{CdS}$ nanocrystals by hydrolyzing polyphosphonic acid anhydride. Our results explain the difference in the nanorod morphology 
between the samples prepared using $\mathrm{CdMe}_{2}$ and that using $\mathrm{CdO}$. It also provided the first example of rational design of reaction condition to control the growth of CdSe and $\mathrm{CdS}$ nanocrystals. 


\section{References}

(1) Peng, X. G.; Manna, L.; Yang, W. D.; Wickham, J.; Scher, E.; Kadavanich, A.; Alivisatos, A. P. Nature 2000, 404, 59-61.

(2) Manna, L.; Scher, E. C.; Alivisatos, A. P. J. Am. Chem. Soc. 2000, 122, $12700-12706$.

(3) Peng, Z. A.; Peng, X. G. J. Am. Chem. Soc. 2001, 123, 1389-1395.

(4) Peng, Z. A.; Peng, X. G. J. Am. Chem. Soc. 2002, 124, 3343-3353.

(5) Puzder, A.; Williamson, A. J.; Zaitseva, N.; Galli, G.; Manna, L.; Alivisatos, A. P. Nano Lett. 2004, 4, 2361-2365.

(6) Rempel, J. Y.; Trout, B. L.; Bawendi, M. G.; Jensen, K. F. J. Phys. Chem. $B$ 2006, 110, 18007-18016.

(7) Manna, L.; Wang, L. W.; Cingolani, R.; Alivisatos, A. P. J. Phys. Chem. B 2005, 109, 6183-6192.

(8) Liu, H. T.; Owen, J. S.; Alivisatos, A. P. J. Am. Chem. Soc. 2007, 129, $305-312$

(9) Carbone, L.; Kudera, S.; Carlino, E.; Parak, W. J.; Giannini, C.; Cingolani, R.; Manna, L. J. Am. Chem. Soc. 2006, 128, 748-755.

(10) A. A. Granovsky, PC GAMESS version 7.0, http://classic.chem.msu.su/gran/gamess/index.html. Accessed Jan 2007.

(11) Becke, A. D. J. Chem. Phys. 1993, 98, 5648-5652.

(12) Lee, C. T.; Yang, W. T.; Parr, R. G. Phys. Rev. B 1988, 37, 785-789.

(13) Vosko, S. H.; Wilk, L.; Nusair, M. Can. J. Phys. 1980, 58, 1200-1211. 
(14) Stephens, P. J.; Devlin, F. J.; Chabalowski, C. F.; Frisch, M. J. J. Phys. Chem. 1994, 98, 11623-11627.

(15) Basis sets were obtained from the Extensible Computational Chemistry Environment Basis Set Database, Version 02/02/06, as developed and distributed by the Molecular Science Computing Facility, Environmental and Molecular Sciences Laboratory which is part of the Pacific Northwest Laboratory, P.O. Box 999, Richland, Washington 99352, USA, and funded by the U.S. Department of Energy. The Pacific Northwest Laboratory is a multi-program laboratory operated by Battelle Memorial Institute for the U.S. Department of Energy under contract DE-AC06-76RLO 1830. Contact Karen Schuchardt for further information.

(16) Check, C. E.; Faust, T. O.; Bailey, J. M.; Wright, B. J.; Gilbert, T. M.; Sunderlin, L. S. J. Phys. Chem. A 2001, 105, 8111-8116.

(17) Hehre, W. J.; Ditchfie, R.; Pople, J. A. J. Chem. Phys. 1972, 56, 2257.

(18) Li, L. S.; Hu, J. T.; Yang, W. D.; Alivisatos, A. P. Nano Lett. 2001, 1, 349-351. 


\section{Chapter 6}

\section{Preparation of Asymmetric Nanostructures from CdTe Tetrapod Nanocrystals}

Reproduced with permission from "Liu, H.; Alivisatos, A. P. "Preparation of Asymmetric Nanostructures through Site Selective Modification of Tetrapods" Nano Letter 2004, 4, 2397-2401, Copyright 2004 American Chemical Society.

\subsection{Introduction}

In the previous chapters, I have discussed the chemistry of group II-VI nanocrystal synthesis from molecular precursors. The synthesis of nanocrystal can be viewed as a self-assemble process by which small building blocks, i.e., atoms, are assembled into a super-structure, i.e., nanocrystal. In some aspects, nanocrystal itself also mimics some properties of atom. One of the most often cited examples is the analogy between quantum dots and atoms, both having discrete energy levels in their electronic structures. In fact, quantum dots are often called 'artificial atoms' for this reason. In the field of nanoscience, to build self-assembled structures from nanocrystals has been an area of active research. The ability to build controlled nanocrystal assemblies, or “artificial molecules", from nanocrystal "artificial atoms"1-3 makes it possible to mimic 
the molecular world in a much larger dimension. State of the art nanomaterial syntheses can now produce colloidal nanoparticles (NPs) with controlled sizes, shapes, and compositions ${ }^{4,5}$. However, only highly symmetric NPs, such as spheres ${ }^{6}, \operatorname{rods}^{7,8}$, and more recently tetrapods ${ }^{9}$ have been well studied. Nanometer sized materials of greater complexity can be built with components of lower symmetry, offering the possibility of creating materials with a higher level of integrated functionality.

Asymmetry is a common feature of the building blocks in many self-assembled structures. For example, phospholipids, molecules that have polar groups on one end and non-polar ones on the other, are basic building blocks for cell membranes. The asymmetry in hydrophilicity enables them to self- assemble into bilayer structures in water. Another very good example is diblock copolymer, which can self-assemble into different morphologies such as spheres, cylinders, and lamellae. In this case, the selfassembly depends not only on the existence of asymmetry, i.e. the two different blocks, but also on the degree of asymmetry, which is the volume ratio between them.

Asymmetric nanostructures are more versatile building blocks compared to their symmetric counterparts. For example, when a gold NP is asymmetrically modified with only one single strand DNA (ssDNA), it can be used as the building block to prepare more complex structures such as dimers and trimers ${ }^{1,3}$. Recently, it has been shown that asymmetric diblock Au-polymer nanorods can self-assemble into bundles, tubes, and sheets ${ }^{10}$. The degree of asymmetry of the nanorods, which is the length ratio of the two blocks, determines the final assembled structure, similar to the case of the diblock copolymer. 
Some asymmetric structures have been prepared using post synthesis modifications. For example, gold half-shell structures were produced by evaporating gold onto an array of silica colloidal particles ${ }^{11}$. Heat treatment of these structures gave goldmetal oxide asymmetric dimers ${ }^{12}$. In a similar approach, gold shell structures have been grown from Au NP seeds on a silica surface to produce gold cups or caps ${ }^{13}$. Direct synthesis of asymmetric structures has also been realized in nanowire systems using chemical vapor deposition ${ }^{14-16}$. Similarly, asymmetric rods can be prepared using template directed growth ${ }^{10,17}$.

In many of the above examples, relatively large particles were prepared. For example, the gold-metal oxide asymmetric dimers can only be prepared from metal oxide particles larger than $200 \mathrm{~nm}$. The gold-polymer asymmetric nanorods have diameters around $200 \mathrm{~nm}$ and lengths over one micrometer. As a result, they can only be used to prepare assemblies in micrometer or even larger dimensions. To prepare sub-micrometer sized assemblies, smaller asymmetric building blocks are needed. Furthermore, it is desirable to develop a range of methods that can yield such structures.

CdTe tetrapods were recently prepared in high yield in our group ${ }^{9}$. The structure of the tetrapod is topologically similar to that of a $s p^{3}$ hybridized carbon atom. With the possibility to mimic the bonding between carbon atoms in organic molecules, this type of structure can serve as a very interesting building block to prepare superstructures, especially three dimensional ones. For example, it would be very interesting to modify each of the four arms differently, to produce a chiral "nanocrystal molecule". Another advantage of using tetrapods as building blocks lies in the fact that the dimension of tetrapods can be tuned over a wide range. For example, the arm length can be made from 
less then $20 \mathrm{~nm}$ to over $200 \mathrm{~nm}$, while keeping the arm diameter unchanged. This feature makes it possible to use tetrapods to prepare structures over a wide range of sizes and complexities.

In this chapter, I demonstrate the first step toward this direction: the preparation of asymmetric tetrapods. I show that it is possible to modify only one of the four arms of the tetrapod with $\mathrm{Au}$ NPs. It is further shown that asymmetrically modified $\mathrm{CdTe}$ nanorods can be produced by breaking this structure. The structures prepared here are potential building blocks to more complex assemblies. The tetrapod arms were modified with gold NPs instead of small molecules, mainly for the ease of visualization. It is difficult to characterize organic species on the semiconductor NP surface with nanometer spatial resolution using current instrumentation. However, use of NPs as tags allows us to use transmission electron microscopy (TEM) or scanning electron microscopy (SEM) to directly image the modified structures. In addition, the surface chemistry of gold is well developed. Attaching gold particles to tetrapods provides a surface with unique chemical properties which can be used for further functionalization or preparation of nanostructure assemblies. Finally, Banin and coworkers have recently prepared CdSe nanorods and tetrapods with single Au nanoparticles directly attached to the ends ${ }^{18}$. It should be of interest to compare the properties of those systems with the asymmetric ones produced here.

\subsection{Results and Discussions.}

In order to selectively modify only one of the four arms, the approximate $T_{d}$ symmetry of the tetrapods must be broken first. This was realized by putting tetrapods 
onto a flat silicon surface. Because of the unique geometry of the tetrapods, three arms contacted with the surface and the last one pointed away from the surface. To produce high density tetrapod films with minimum aggregation, spin-coating and LangmuirBlodgett techniques were first tested but both produced a high degree of aggregation. To our surprise, slow evaporation of tetrapod solutions in pyridine produced uniform tetrapod coverage with almost no aggregation over the range of $100 \mu \mathrm{m}$ (Figure 6-1).

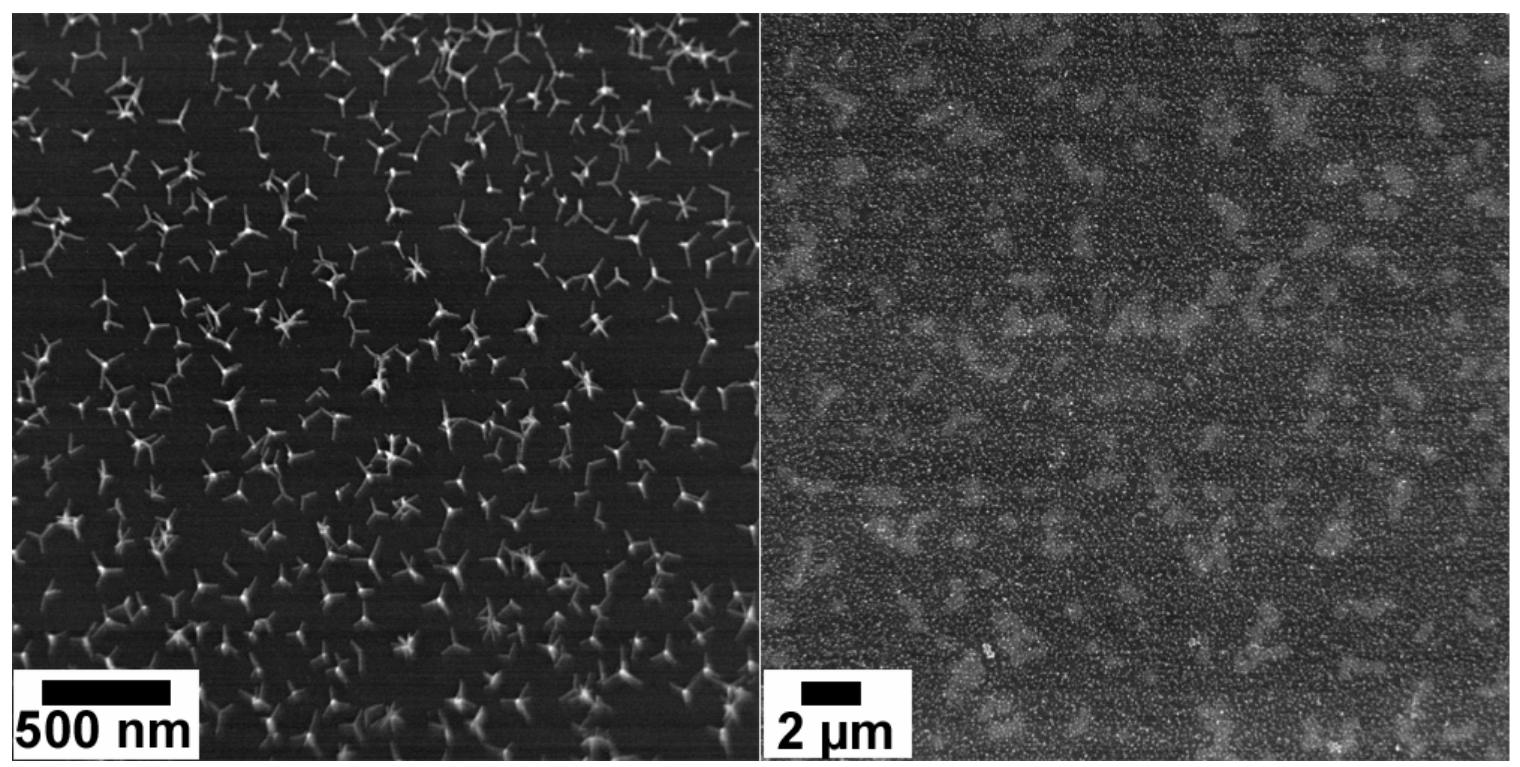

Figure 6-1. SEM images (top down view) of the prepared tetrapod film on Si wafer. Left: High resolution image showing individual tetrapods. The top arms appear as bright spots. Right: Low resolution image showing the large area homogenous film. Each tiny white spot corresponds to one tetrapod top arm. The large white islands result from organic contamination

To achieve modification of only one arm of the tetrapods, a polymer film was then spin-coated to cover the bottom three arms, exposing only part of the top arm 
(Scheme 6-1, step A). So far, two polymers have been tested: poly (3-hexyl-thiophene) (P3HT) and poly (methyl methacrylate) (PMMA). Because P3HT is a semiconductor polymer, SEM can be used to characterize the surface before and after spin-coating. After $\mathrm{a} \sim 40 \mathrm{~nm}$ thick P3HT layer was spin-coated, the bottom three arms showed reduced contrast in SEM images, which suggested that they were covered by the polymer film (data not shown). Atomic force microscopy (AFM) was used to further characterize the composite. Figure 6-2 shows the height image of the polymer-tetrapod surface. Separated dots can be found, which correspond to the exposed top arms. The heights of the dots roughly match the estimated exposed arm lengths. 


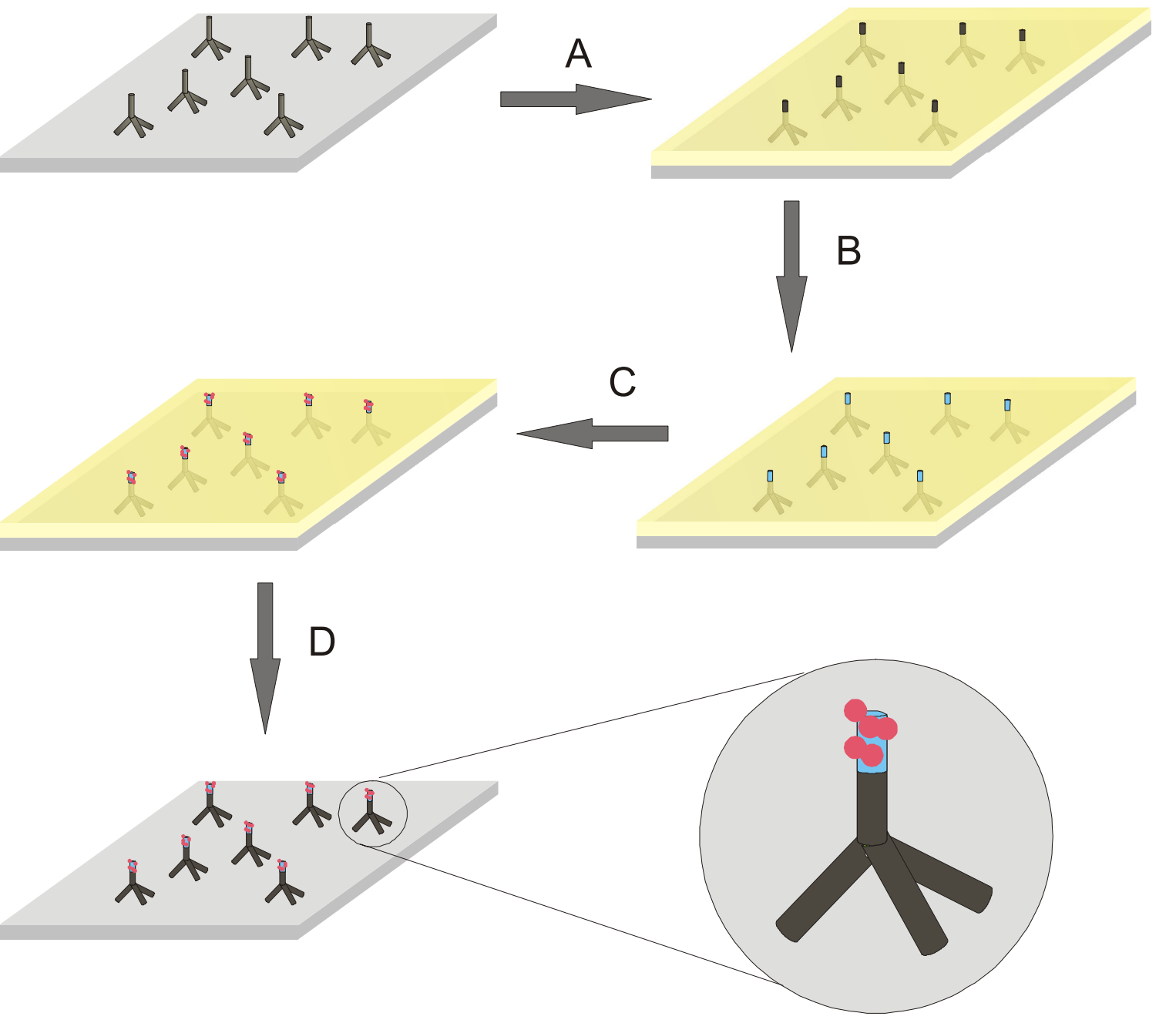

Scheme 6-1. Preparation of asymmetric tetrapods. A: Spin-coat polymer to partially cover tetrapods. B: Treat with hexanedithiol solution. Hexanedithiol modified surface is shown in blue color. C: Attach gold NP (shown as red dots) onto modified tetrapod surface. D: Dissolve the polymer layer. 

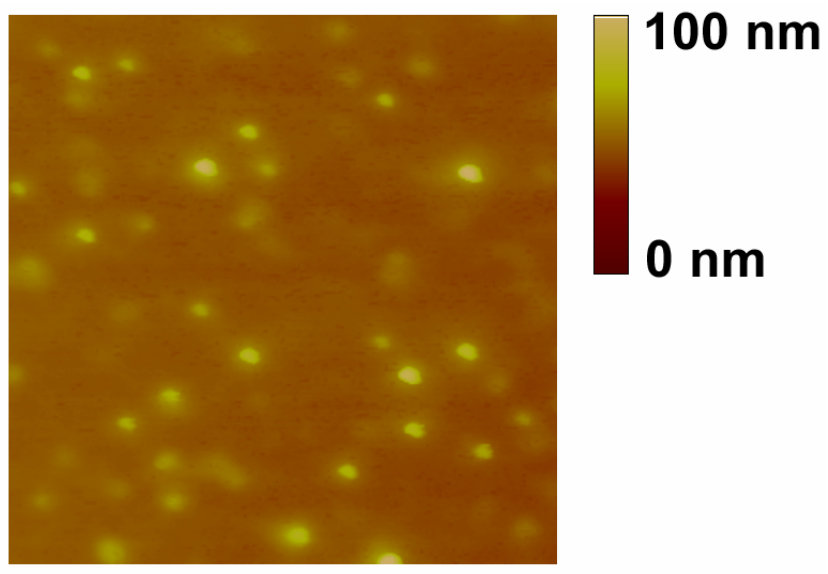

Figure 6-2. Tapping mode AFM height image of the tetrapods partially covered with polymer. The image size is $1 \mu \mathrm{m} \times 1 \mu \mathrm{m}$. The high spots correspond to exposed top arms.

The composite film was then treated with an isopropanol solution of hexanedithiol overnight to modify the exposed top arms (Scheme 6-1, step B). P3HT and PMMA are not soluble in isopropanol so the bottom three arms remained covered throughout this process. Thiol groups readily bind to the CdTe surface. In the case of hexanedithiol, one thiol group binds to the CdTe surface while the other one remains free. Gold NPs coated with trioctylphosphine (TOP) were then used to modify the top arms via the dithiol linkers (Scheme 6-1, step C) ${ }^{19}$. TOP coated NPs instead of thiol coated ones were used because TOP can be easily replaced by the thiol groups. The diameters of the gold NPs and arms of the tetrapod we used here were around $5 \mathrm{~nm}$ and $10 \mathrm{~nm}$, respectively. As a result, multiple gold NPs bound to the top arms in this step. After the modification, the polymer film was removed by dissolving it in organic solvent such as chloroform (for P3HT) or pyridine (for PMMA) to expose the whole structure (Scheme 6-1, step D). 
SEM and TEM were used to characterize the prepared asymmetric tetrapods. Figure 6-3 shows the SEM images of the resulting asymmetric tetrapods using PMMA as the protecting layer. It can be clearly seen that the tips of the top arms have larger diameters compared with the bottom parts, as a result of the attachment of large numbers of Au NPs. The fine structure is revealed in the selected higher resolution images, shown at the bottom of figure 6-3. Individual gold NPs can be seen on the top arm while the other three arms are clean. Similar results were obtained using P3HT as the protecting polymer.

Control experiments demonstrate that the modification is indeed mediated by the dithiol linker instead of nonspecific adsorption. A silicon chip was spilt into two halves after deposition of tetrapods and spin-coating of the P3HT film. One of the pieces was treated with hexanedithiol solution and the other was not. Both of them were then treated with Au NPs and the polymer layers were removed afterwards. Shown in figure 6-4, the sample without hexanedithiol treatment shows no binding of gold NPs while the other one shows successful asymmetric modification.

In one experiment, two cycles of hexanedithiol and gold NP modifications were performed before the polymer protecting layer was removed. This procedure produced two layers of gold NP shells on the exposed top arms. A much larger cluster of gold NPs can be seen on the top arms (Figure 6-5). In a different approach, the thickness of the polymer layers was varied to control the exposed arm length in order to control the degree of modification (data not shown). 


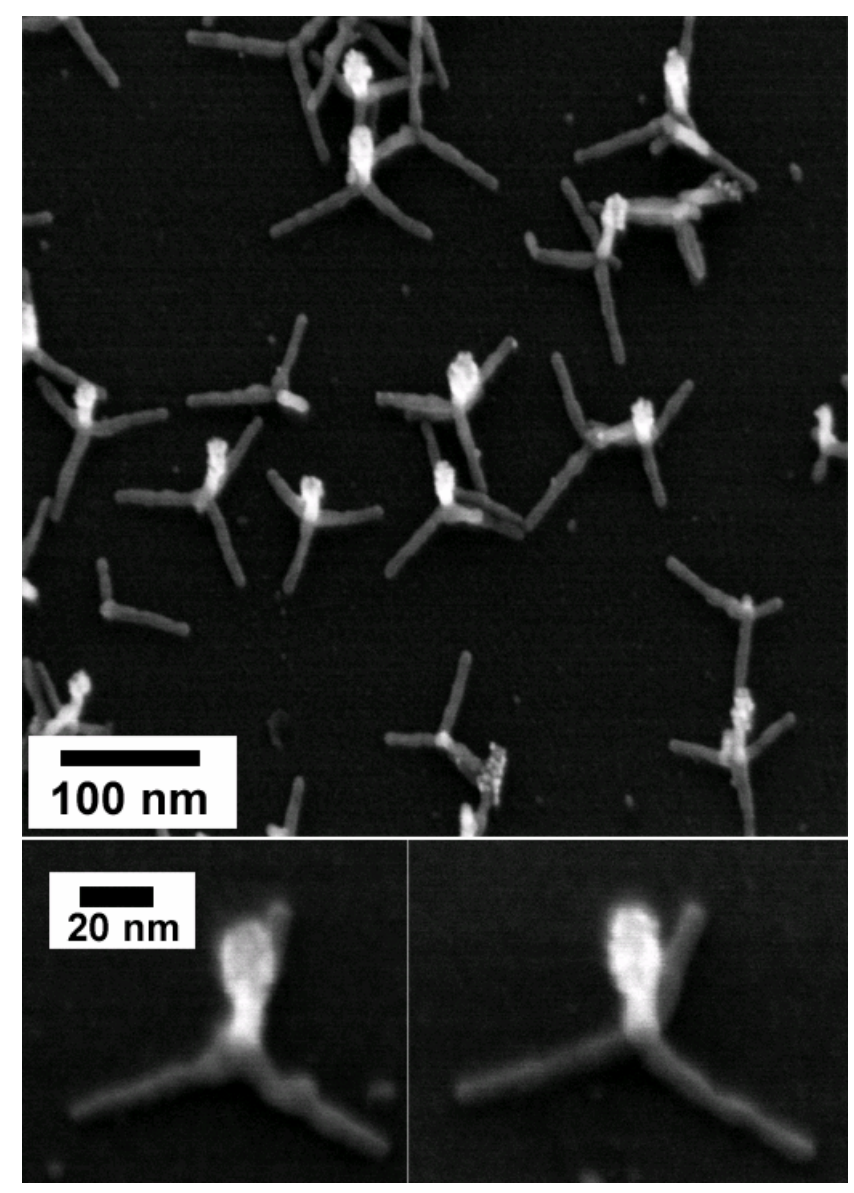

Figure 6-3. SEM images (tilted 30 degrees) of as prepared asymmetric tetrapods on $\mathrm{Si}$ surface. The top arms were modified with many gold NPs. Top: large area view. Bottom: selected high resolution images.

It is desirable to release the modified tetrapods intact from the substrate. So far this has not proven possible, because of the very strong interaction with the substrate. When the tetrapods are first deposited, the capillary force from solvent evaporation slams the tetrapods onto the surface, flattening the three arms that are pointing down towards the substrate. This effect has been visualized previously in experiments where the tetrapods were deposited to the side walls of trenches and could be viewed from the 
side. ${ }^{20}$ It also has been seen by C. Kieselowski for tetrapods deposited on flat surfaces using tomographic reconstruction in the TEM. ${ }^{21}$ In the future, it may be possible to gently deposit the tetrapods using supercritical fluid, to avoid strong capillary forces. In the meantime, we have learned how to break off the uppermost arm of the tetrapod, which is asymmetrically modified with Au NPs.

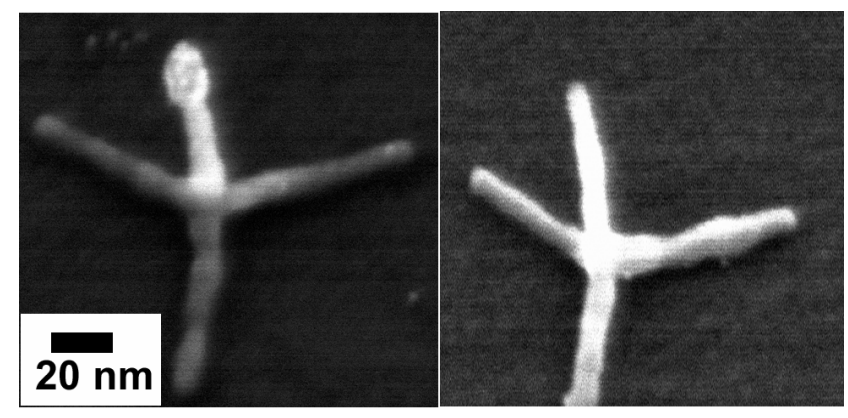

Figure 6-4. Control experiment result supports that the modification is a chemical one. Left: SEM image of CdTe tetrapods obtained after treating with hexanedithiol and Au NPs. Right: SEM image of CdTe tetrapod obtained after treating with Au NPs only. The tetrapods were not modified with Au NPs in this case.

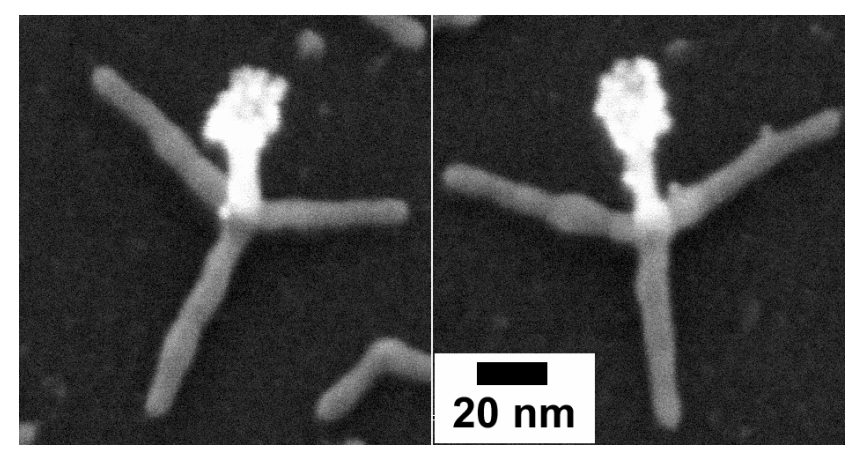

Figure 6-5. SEM images of asymmetric tetrapods prepared using two cycles of hexanedithiol, Au NP modifications. There are much more Au NPs on the top arm compared to those in the figure 6-3. 
This was accomplished by pressing a TEM grid onto the silicon surface (Scheme 6-2). During this process, particles were transferred to the TEM grid. Indeed, we were able to find a few asymmetrically modified tetrapods on some rare occasions (Figure 66). However, to our surprise, the majority of the materials on the TEM grid were nanorods (Figure 6-7, top left). Under higher magnification, it can be seen that the nanorods were asymmetrically modified with gold NPs (Figure 6-7, top right) formed by breaking the modified top arms by the TEM grid. We found that this break-and-transfer process could be applied to other substrates as well. The bottom of Figure 6-7 shows SEM images of the broken asymmetric CdTe nanorods transferred to a Si substrate. We want to emphasize here that this simple method produces asymmetric nanorods which are very hard to prepare in other ways. Besides the interesting semiconductor-metal heterostructure itself, these asymmetric nanorods can also be used as useful basic building blocks. For example, if functionalized by appropriate organic molecules on the gold NPs; they can be potentially made into nanocrystal amphilphiles. Furthermore, they should exhibit anomalously large transient electric birefringence ${ }^{22}$, and may have interesting non-linear optical properties.
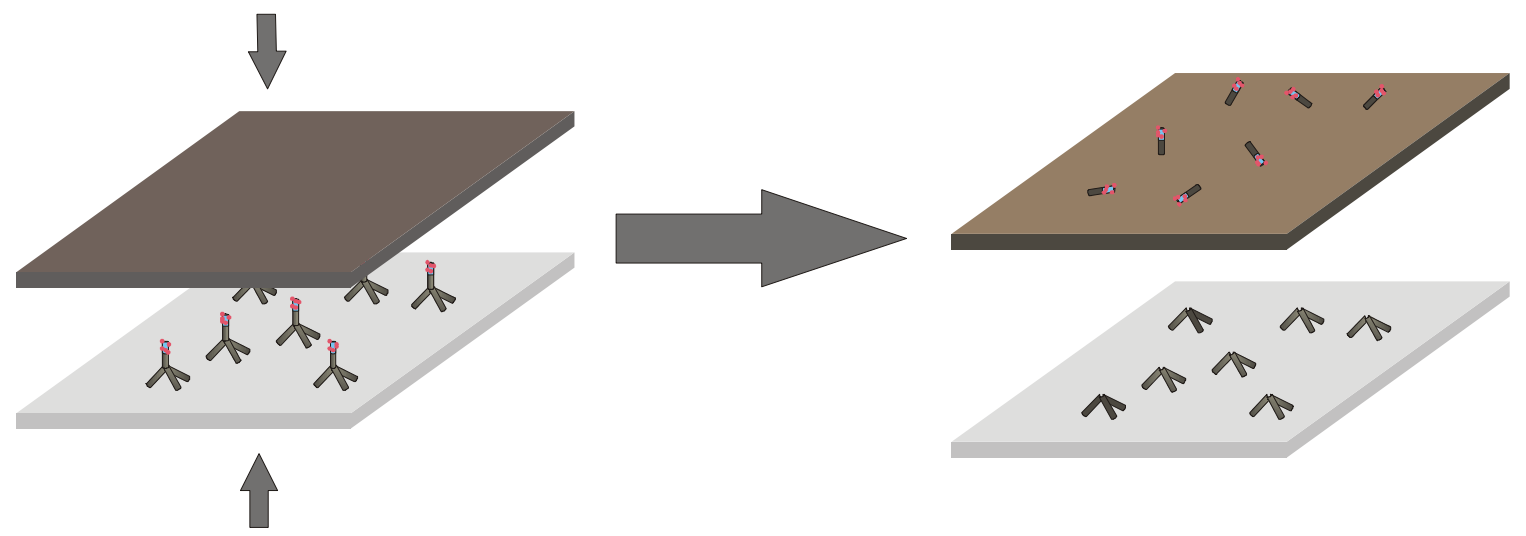

Scheme 6-2. Asymmetric tetrapods are broken to produce asymmetric rods. 


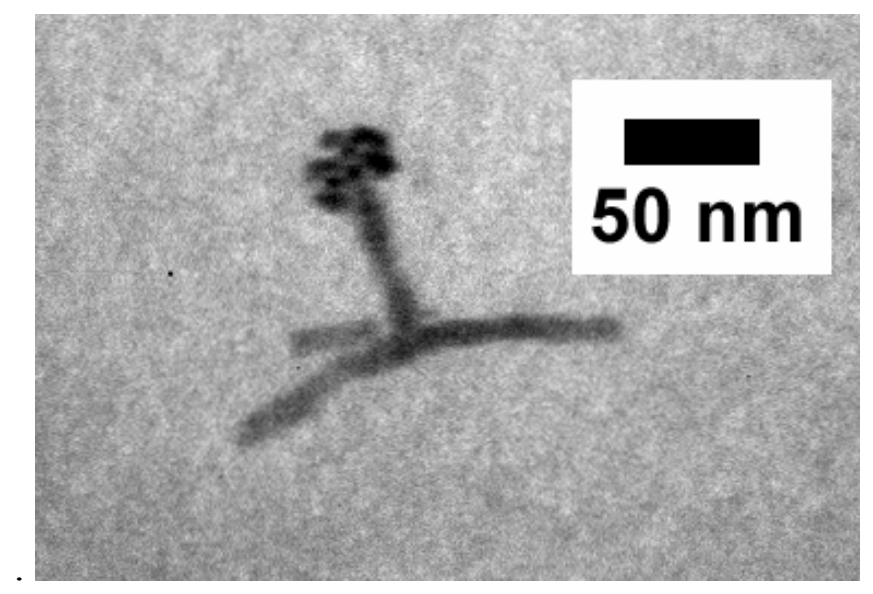

Figure 6-6. TEM image of asymmetric tetrapod.

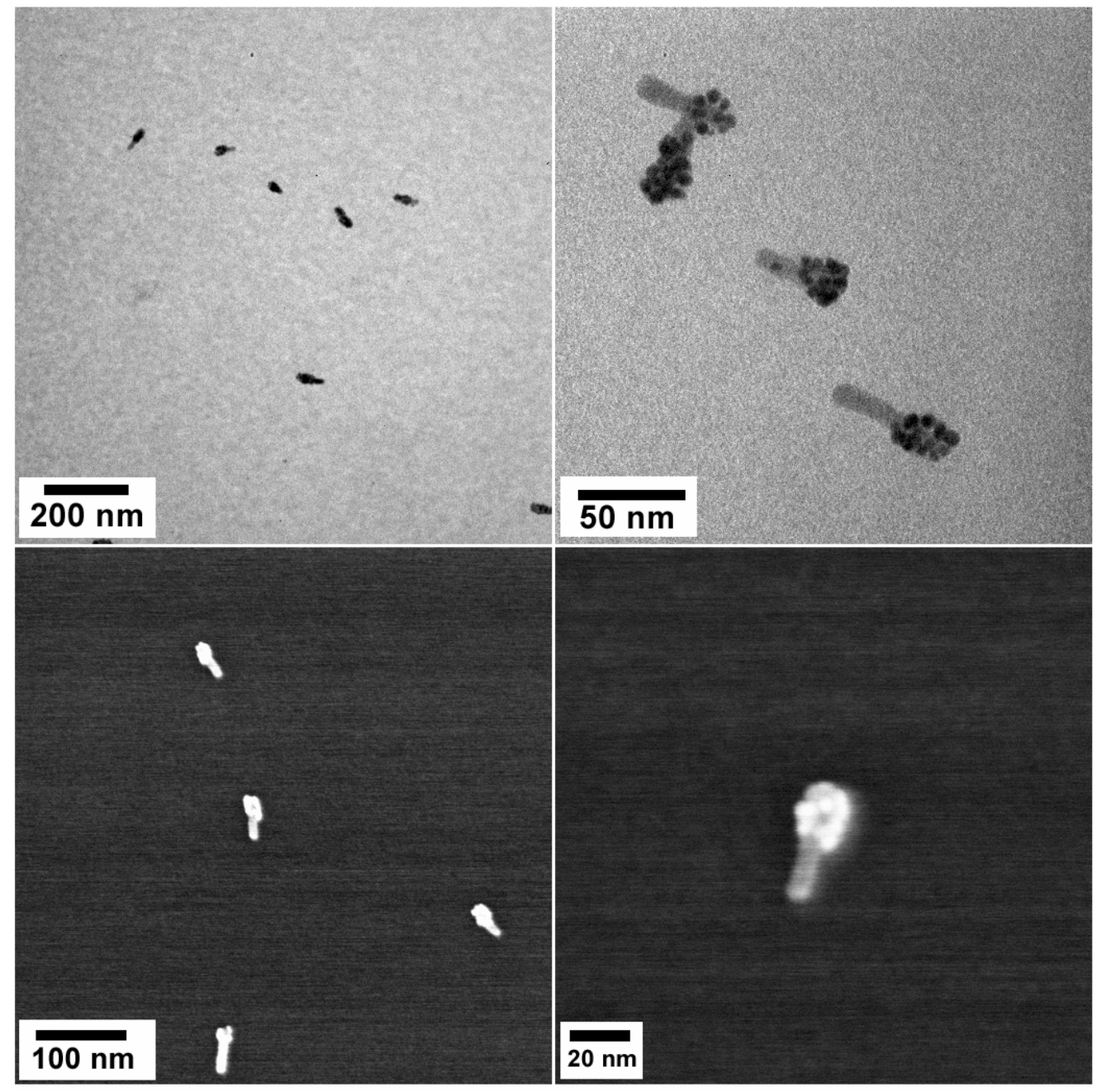


Figure 6-7. TEM (top) and SEM (bottom) images of asymmetrically modified CdTe rods

In conclusion, we successfully prepared asymmetrically modified CdTe tetrapods and nanorods via a site selective modification method. These novel structures can be potentially used as basic building blocks to prepare more complex nanostructures. The mild preparation process makes it possible to modify other tetrapod shaped substrates, for example, $\mathrm{CdSe}^{8}$ and $\mathrm{ZnO}$ tetrapods ${ }^{23}$ in the future. By using different material specific linker molecules, other NPs can also be used in the modification.

\section{References}

(1) Alivisatos, A. P.; Johnsson, K. P.; Peng, X. G.; Wilson, T. E.; Loweth, C. J.; Bruchez, M. P.; Schultz, P. G. Nature 1996, 382, 609-611.

(2) Peng, X. G.; Wilson, T. E.; Alivisatos, A. P.; Schultz, P. G. Angew. Chem. Int. Ed. Engl. 1997, 36, 145-147.

(3) Loweth, C. J.; Caldwell, W. B.; Peng, X. G.; Alivisatos, A. P.; Schultz, P. G. Angew. Chem. Int. Ed. Engl. 1999, 38, 1808-1812.

(4) Daniel, M. C.; Astruc, D. Chem. Rev. 2004, 104, 293-346.

(5) Crouch, D.; Norager, S.; O'Brien, P.; Park, J. H.; Pickett, N. Philos. T. Roy. Soc. A 2003, 361, 297-310.

(6) Murray, C. B.; Norris, D. J.; Bawendi, M. G. J. Am. Chem. Soc. 1993, $115,8706-8715$. 
(7) Peng, X. G.; Manna, L.; Yang, W. D.; Wickham, J.; Scher, E.; Kadavanich, A.; Alivisatos, A. P. Nature 2000, 404, 59-61.

(8) Manna, L.; Scher, E. C.; Alivisatos, A. P. J. Am. Chem. Soc. 2000, 122, 12700-12706.

(9) Manna, L.; Milliron, D. J.; Meisel, A.; Scher, E. C.; Alivisatos, A. P. Nature Mater. 2003, 2, 382-385.

(10) Park, S.; Lim, J. H.; Chung, S. W.; Mirkin, C. A. Science 2004, 303, 348351.

(11) Love, J. C.; Gates, B. D.; Wolfe, D. B.; Paul, K. E.; Whitesides, G. M. Nano Lett. 2002, 2, 891-894.

(12) Lu, Y.; Xiong, H.; Jiang, X. C.; Xia, Y. N.; Prentiss, M.; Whitesides, G. M. J. Am. Chem. Soc. 2003, 125, 12724-12725.

(13) Charnay, C.; Lee, A.; Man, S. Q.; Moran, C. E.; Radloff, C.; Bradley, R. K.; Halas, N. J. J. Phys. Chem. B 2003, 107, 7327-7333.

(14) Wu, Y. Y.; Fan, R.; Yang, P. D. Nano Lett. 2002, 2, 83-86.

(15) Gudiksen, M. S.; Lauhon, L. J.; Wang, J.; Smith, D. C.; Lieber, C. M. Nature 2002, 415, 617-620.

(16) Bjork, M. T.; Ohlsson, B. J.; Sass, T.; Persson, A. I.; Thelander, C.; Magnusson, M. H.; Deppert, K.; Wallenberg, L. R.; Samuelson, L. Nano Lett. 2002, 2, 87-89. 
(17) Salem, A. K.; Searson, P. C.; Leong, K. W. Nature Mater. 2003, 2, 668671.

(18) Mokari, T.; Rothenberg, E.; Popov, I.; Costi, R.; Banin, U. Science 2004, 304, 1787-1790.

(19) The TOP coated Au NP was synthesized by reducing $\mathrm{HAuCl}_{4}$ in the presence of $\mathrm{TOP} . \mathrm{HAuCl}_{4}(97.6 \mathrm{mM}$ in water, $1 \mathrm{~mL})$ was mixed with tetraoctyl ammonium bromide $(0.1 \mathrm{M}$ in toluene, $4 \mathrm{~mL})$ and toluene $(4 \mathrm{~mL})$. The mixture was stirred until the gold salt transferred to the toluene layer. The organic layer was then separated and dried using molecular sieve. The dried solution $(1 \mathrm{~mL})$ was mixed with toluene $(4 \mathrm{~mL})$ and TOP $(20 \mu \mathrm{L})$. Lithium triethyl borohydride $(1 \mathrm{M}$ in THF, $1 \mathrm{~mL})$ was injected into the mixture and the reaction was stirred in dark. The NP was precipitated by adding methanol. After separating the precipitate from the supernatant, it was redissolved in toluene. The precipitation - redispersion was then repeated once. The NP solution was diluted with isopropanol (1 : 10 by mass) and used in the tetrapod modification immediately.

(20) Cui, Y.; Bjork, M. T.; Liddle, J. A.; Sonnichsen, C.; Boussert, B.; Alivisatos, A. P. Nano Lett. 2004, 4, 1093-1098.

(21) Kieselowski, C. Private Communication

(22) Li, L. S.; Alivisatos, A. P. Phys. Rev. Lett. 2003, 90, 097402.

(23) Yan, H.; He, R.; Pham, J.; Yang, P. Adv. Mater. 2003, 15, 402-405. 


\section{Chapter 7}

\section{Conclusion and Outlook}

\subsection{Summary of Research}

This dissertation studied the chemistry of the group II-VI colloidal nanocrystal synthesis based on the approach of high temperature decomposition of precursors. By using a combination of NMR and MS techniques, I have fully characterized the molecular structures of the precursors and products of the synthesis of cadmium and zinc chalcogenides nanocrystals. ${ }^{31} \mathrm{P}$ NMR study suggests that chalcogen precursor is activated by binding to the metal precursor using the chalcogen atom. Nucleophilic attack on the activated phosphine chalcogenide cleaves the phosphorous - chalcogen bond and releases the chalcogen atom for nanocrystal growth.

Density functional theory calculation was used to map the detailed free energy landscape of the precursor to monomer conversion pathway for the synthesis of $\mathrm{CdSe}$ nanocrystal from cadmium carboxylate and phosphine selenide. The results show that phosphine selenide binds to cadmium carboxylate using the selenium atom. The phosphorous atom of the phosphine selenide was attack by a carboxylate to give an intermediate, converting the $\mathrm{P}=\mathrm{Se}$ double bond to a $\mathrm{P}$-Se single bond in the process. This intermediate then binds to a carboxylic acid surfactant molecule and breaks the P-Se single bond via a proton transfer from the carboxylic acid to the Se atom. These results 
show that surfactant molecule plays an important role in the cleavage of phosphine selenide and is an integral part of the nanocrystal synthesis.

Based on the above experimental and theoretical results, I have designed a new synthetic method that uses water as a reagent to control the growth of CdSe and $\mathrm{CdS}$ nanorod in the diameter dimension. An analysis of the CdSe and CdS nanocrystal synthesis shows that poly-phosphonic acid was produced as a major reaction product when alkyl-phosphonic acid was used as the surfactant. Poly-phosphonic acid is a polydentate ligand that should bind to nanocrystal surface stronger than regular phosphonic acid does, which is verified by density functional theory calculations. The fact that polyphosphonic acid readily hydrolyzes in the presence of water was used to control the degree of surface passivation of nanocrystal and thus the nanocrystal growth kinetics. Experimental results quantitatively reproduced our theoretical predictions.

Finally, the asymmetric modification of CdTe tetrapod nanocrystal using $\mathrm{Au}$ nanocrystal was described. The structure of the tetrapod is topologically similar to that of a $s p^{3}$ hybridized carbon atom, which makes it an attractive candidate to prepare selfassembled structures. CdTe tetrapods were deposited on a substrate, and partially coated with a protective polymer layer, exposing just one arm. The exposed arm was then decorated with $\mathrm{Au}$ nanoparticles in a site selective fashion. The modified arms were readily broken off from the remainder of the tetrapods, and released from the substrate, yielding CdTe nanorods asymmetrically modified with Au nanoparticles. 


\subsection{Outlook}

Previous study on the synthesis of nanocrystals has been heavily focused on empirical optimization of reaction conditions in order to obtain high quality nanocrystals. Little effort has been made to understand the underlying mechanism that produces the nanocrystal in the first place. Work presented in this dissertation represents one of the first attempts toward this goal.

Synthesis of nanocrystal from its precursors is a rather complicated process that involves monomer formation, nucleation, and finally nanocrystal growth. This dissertation only discussed the precursor decomposition process, which presumably also generates monomer. The mechanism of nanocrystal nucleation and growth would be the natural extension of the current study. However, experimental study of this kind would be rather difficult since structural characterization of monomer and nuclei remains a challenge. Computer simulation, on the other hand, is not restrained by these problems and has the potential of providing detailed mechanistic information on the nucleation and growth of nanocrystals.

Nevertheless, the results presented in this dissertation, though limited in scope, can still be successfully used to improve the understanding of current nanocrystal synthesis and hopefully, develop totally new synthetic methods. As an example, I presented a mechanism based design of nanorod synthesis that uses a very unconventional reagent, water, to control the growth of nanorod. Future work in this direction will certainly provide more examples to showcase the power of chemistry in creating novel materials. 